\title{
The pressure of deconfined QCD for all temperatures and quark chemical potentials
}

\author{
A. Ipp \\ ECT*, Villa Tambosi, Strada delle Tabarelle 286, \\ I-38050 Villazzano Trento, Italy \\ K. Kajantie \\ Department of Physics, P.O. Box 64, FI-00014 University of Helsinki, Finland
}

A. Rebhan

Institut für Theoretische Physik, Technische Universität Wien, Wiedner Hauptstr. 8-10, A-1040 Vienna, Austria

\section{A. Vuorinen}

Department of Physics, University of Washington, Seattle, WA 98195, U.S.A.

We present a new method for the evaluation of the perturbative expansion of the QCD pressure which is valid at all values of the temperature and quark chemical potentials in the deconfined phase and which we work out up to and including order $g^{4}$ accuracy. Our calculation is manifestly four-dimensional and purely diagrammatic - and thus independent of any effective theory descriptions of high temperature or high density QCD. In various limits, we recover the known results of dimensional reduction and the HDL and HTL resummation schemes, as well as the equation of state of zero-temperature quark matter, thereby verifying their respective validity. To demonstrate the overlap of the various regimes, we furthermore show how the predictions of dimensional reduction and HDL resummed perturbation theory agree in the regime $T \sim \sqrt{g} \mu$. At parametrically smaller temperatures $T \sim g \mu$, we find that the dimensional reduction result agrees well with those of the nonstatic resummations down to the remarkably low value $T \approx 0.2 m_{\mathrm{D}}$, where $m_{\mathrm{D}}$ is the Debye mass at $T=0$. Beyond this, we see that only the latter methods connect smoothly to the $T=0$ result of Freedman and McLerran, to which the leading small- $T$ corrections are given by the so-called non-Fermiliquid terms, first obtained through HDL resummations. Finally, we outline the extension of our method to the next order, where it would include terms for the low-temperature entropy and specific heats that are unknown at present.

PACS numbers: 11.10.Wx, 12.38.Mh 


\section{Contents}

\begin{tabular}{ll} 
I. Introduction & 3 \\
\hline
\end{tabular}

A. The notation $\quad 5$

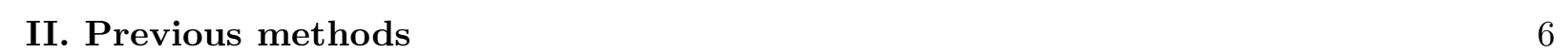

A. Dimensional reduction 6

B. The Freedman-McLerran result for the $T=0$ pressure 8

C. Minimal HTL/HDL resummation $\quad 9$

1. $T$ parametrically smaller than $m_{\mathrm{D}} \quad 10$

2. $T$ parametrically larger than $m_{\mathrm{D}} \quad 11$

III. The new approach 12

A. Identification of the relevant diagrams 12

$\begin{array}{ll}\text { B. The 2GI diagrams } & 13\end{array}$

C. The ring sum 14

$\begin{array}{ll}\text { D. The double and triple sums } & 16\end{array}$

$\begin{array}{ll}\text { E. The result } & 18\end{array}$

$\begin{array}{ll}\text { F. Numerical infrared issues } & 19\end{array}$

\begin{tabular}{|lr}
\hline IV. Numerical results & 20
\end{tabular}

A. $T$ parametricallv larger than $m_{D} \quad 20$

B. $T$ comparable to $m_{\mathrm{D}} \quad 22$

C. $T$ smaller than $m_{\mathrm{D}} \quad 26$

V. Summary of perturbation theory on the $\mu$ - $T$ plane 29

\begin{tabular}{|l|l|}
\hline VI. Conclusions and outlook & 32
\end{tabular}

$\begin{array}{ll}\text { Acknowledgments } & 33\end{array}$

A. Dimensional reduction result at finite $T$ and $\mu$

B. The analytic values of various parts of the pressure $\quad 35$

1. The Vac-Vac and Vac-Mat diagrams

2. The ordinarv ring sum $\quad 35$

3. Small and large $\mu / T$ limits of $p_{\text {and }} \quad 36$

C. Properties of the one-loop gluon polarization tensor

D. Numerical evaluation of $p_{\text {finite }}$

1. Euclidean space contributions

a. High momentum expansion in Euclidean space 40

b. IR mass in Euclidean space $\quad 41$

2. Minkowski space contributions $\quad 41$

a. Contour deformation 41

b. IR mass in Minkowski space

$\begin{array}{ll}\text { References } & 44\end{array}$ 


\section{INTRODUCTION}

The most fundamental thermodynamic quantity in the theory of strong interactions, the QCD pressure $p_{\mathrm{QCD}}(T, \mu)$, can at large values of the temperature $T$ or the quark chemical potentials $\mu$ be computed in a weak coupling expansion in the gauge coupling constant $g$, defined in the $\overline{\mathrm{MS}}$ renormalization scheme. In the region where $T$ is larger than all other relevant mass scales in the problem, the expansion has been extended to include terms of order $g^{6} \log g$ [1]-[8], while at $T=0$ and $\mu$ much greater than the critical chemical potential $\mu_{c}$, the pressure is known up to and including terms of order $g^{4}[9]$. In between these regimes, at $0<T \lesssim g \mu$, anomalous contributions from non-Fermi-liquid behavior have been obtained [10], which involve fractional powers and logarithms of $g$. The purpose of this paper is to connect all these disjoint computations through one expression which gives the pressure at all values of $T$ and $\mu$ up to and including terms of order $g^{4}$.

Ultimately, the reason for the existing results being valid only in the separate domains described above is that different computational methods are practical in different regions of the $\mu-T$ plane. When $T$ is larger than all other dynamical scales, one can, in the spirit of effective theories, integrate out the degrees of freedom corresponding to non-zero Matsubara modes (having thermal masses of order $\pi T$ or higher) to obtain a simpler three-dimensional effective theory. Formally, the requirement for this is that one must have ${ }^{1} T \gg m_{\mathrm{D}}$ which explicitly excludes the region of $T \lesssim g \mu$. At these parametrically lower temperatures, perturbation theory requires a different reorganization which can be most efficiently performed via the hard dense loop (HDL) approximation. This approach corresponds to a different effective field theory which is intrinsically four-dimensional and whose non-local effective action, known only to one-loop order, was found by Braaten, Pisarski, Taylor and Wong [12]. It involves a resummation of nonstatic self-energies which, in fact, was a crucial ingredient already in the classic calculation of Freedman and McLerran 9] for the $\mathcal{O}\left(g^{4}\right)$ zero-temperature pressure. In practice, even before reaching this limit, at $T \sim \mu g^{-5} \exp \left[-3 \pi^{2} /(\sqrt{2} g)\right][13]$, one of course encounters the non-perturbative pairing instability (color superconductivity), but in a strictly perturbative calculation these effects can never be seen.

In view of the fragmented status of the various perturbative results on the $\mu-T$ plane, there is obvious motivation for attempting to develop an independent and uniform method of calculation which would have the power of both verifying the validity of all the existing computations and providing a smooth interpolation between them. Important steps in this direction have already been taken through recent advances in the analytic calculation of sum-integrals at arbitrary temperatures and densities [8, 14] as well as in the numerical evaluation of multi-dimensional integrals involving the one-loop gluon polarization tensor [15]. In fact, these techniques provide all the required machinery for the first purely diagrammatic four-dimensional determination of the QCD pressure up to order $g^{4}$ at arbitrary $\mu$ and $T$ which is what we set out to perform in this work. We take as our starting point the systematic analysis of all relevant classes of Feynman graphs contributing to the partition function up to and including this order which implies that our calculation will be independent of any effective descriptions of the fundamental theory that are valid only in limited

\footnotetext{
${ }^{1}$ Due to the chemical potential $\mu$ appearing in the fermionic propagator with an imaginary unit, this parameter contributes to the long-distance behavior of the free fermionic correlation function (i.e. the Fourier transform of the free propagator) only through an oscillatory phase factor. Therefore, from the point of view of IR physics it can be identified as an irrelevant parameter, whose value only affects the validity of dimensional reduction through the Debye mass scale. Note, however, that this reasoning does not apply to imaginary values of the chemical potential, in particular when its magnitude becomes comparable to $\pi T$. (In this region one is in any case restricted to values $\mu<\pi T / N_{c}$ due to the loss of periodicity in the pressure as a function of imaginary $\mu$ in standard perturbative calculations 11]. This is due to the explicit breaking of the $\mathrm{Z}\left(N_{c}\right)$ symmetry, whose effects are otherwise partially visible even in the presence of dynamical fermions.)
} 
regions of the $\mu-T$ plane.

As is usual in a weak-coupling analysis, we regard the coupling $g$ as an in principle arbitrarily small parameter, in which we set out to expand the pressure around the value $g=0$. In practice, the QCD coupling scale is of course about 1 at $100 \mathrm{GeV}$ and at $1 \mathrm{GeV}$ even about 2, so for our results to be applicable we must assume to be working at sufficiently high temperatures and/or densities. A critical region where the nature of the perturbative expansion of the pressure changes both qualitatively and quantitatively turns out to be that of parametrically small $T \lesssim m_{\mathrm{D}} \sim g \mu$, so we have found it useful to quantify the smallness of $T$ by expressing it in the form $T \sim g^{x} \mu$. We then study the changes that occur when $x$ is increased from zero to values larger than 1 , where the pressure can be re-expanded in a series of (fractional) powers and logarithms of $g$.

Our main results, displayed in Sec. IV include the following. In the region $T / \mu \sim g^{0}$, we explicitly recover the perturbative expansion of the pressure to order $g^{4}$ which was derived in Ref. 8] using dimensional reduction (DR). We show that this result is valid up to arbitrarily high values of the ratio $\mu / T$, verifying the non-trivial behavior of the different perturbative coefficients as functions of the parameter. In deriving these results, we notice that the larger we choose $\mu / T$ to be, the smaller values of $g$ we have to use in the series expansion of our numerical result in order to find agreement with the dimensional reduction one. We interpret this as a demonstration of the fact that dimensional reduction amounts to expanding the pressure in powers of $m_{\mathrm{D}} / T$ on top of $g$ and is thus inapplicable in the region $T \lesssim g \mu$. For temperatures $T \sim \sqrt{g} \mu$, which still fall into the domain of validity of dimensional reduction, we demonstrate the overlap of the different existing results by deriving the same expansion for the pressure (in half-integer powers of $g$ ) from both the perturbative results of Ref. [8] and the HDL resummation scheme of Refs. 10, 16]. At parametrically even smaller temperatures, $T \lesssim g \mu$, we then show how our new calculation - the first one entirely independent of the original one of Freedman and McLerran [9] - smoothly approaches their famous result for the $T=0$ pressure, at the same time reproducing the "anomalous specific heat" contributions to the pressure obtained previously through HDL resummations. In contrast, the dimensional reduction result is observed to agree numerically remarkably well with those of the nonstatic resummations down to $T \approx 0.2 m_{\mathrm{D}}$, or $2 \pi T \approx 1.3 m_{\mathrm{D}}$, but it diverges logarithmically in the limit $T \rightarrow 0$. We explain how this divergence is related to the behavior of the physical, resummed expression for the pressure at large but order $g^{0}$ values of $T /(g \mu)$.

The paper is organized as follows. In Section [I] we review the most important methods applied previously to the evaluation of the QCD pressure, namely dimensional reduction, HTL/HDL resummations and zero-temperature perturbation theory. After this, we present our new scheme in Section III, where we identify the classes of diagrams we need to compute and then write down our new expression for the pressure as the sum of an analytic power series in $g^{2}$ and a logarithmic sum-integral that is to be treated numerically. In Section IV we then finally display and discuss our results. We plot them in various regions of the $\mu-T$ plane as characterized by the exponent $x$ in $T \sim g^{x} \mu$ and compare our predictions to the existing results of dimensional reduction [8] and HTL/HDL resummations [10, 16]. After this, we summarize our findings in Section \. where we explain in a detailed fashion the structure of the perturbative expansion of the QCD pressure on the $\mu-T$ plane, and then draw final conclusions and briefly look into new directions in Section VI Several computational details as well as the explicit analytic forms of many individual contributions to our result are finally displayed in the Appendices where, in particular, Appendix D contains details on the numerical evaluation of the logarithmic sum-integral introduced in Sec. III 


\section{A. The notation}

To conclude the introduction, let us fix our notational conventions which to a large extent follow those of Ref. [8]. First, throughout the text we will be working in the Feynman gauge, in which many of the existing results our calculation relies on have been derived. The chemical potentials of the different flavors, for which we apply the notation

$$
\bar{\mu} \equiv \frac{\mu}{2 \pi T}, \quad z \equiv 1 / 2-i \bar{\mu},
$$

are assumed to be equal, and thus we suppress all subscripts for them. (The renormalization scale will be denoted by the symbol $\bar{\Lambda}$ and should not be confused with the above $\bar{\mu}$.) For the analytic result of Eq. (3.20), the generalization to several independent chemical potentials is trivial and only requires the multiplication of the results by $1 / N_{f} \sum_{f}$, but for the outcome of the numerical calculation, this is less straightforward. All of our methods can naturally be applied to any combination of $T$ and the $\mu_{f}$ 's, but this will have to be done on a case-by-case basis.

As usual, we denote the most common group theory constants by

$$
\begin{aligned}
C_{A} \delta^{c d} & \equiv f^{a b c} f^{a b d}=N \delta^{c d}, \\
C_{F} \delta_{i j} & \equiv\left(T^{a} T^{a}\right)_{i j}=\frac{N^{2}-1}{2 N} \delta_{i j}, \\
T_{F} \delta^{a b} & \equiv \operatorname{Tr} T^{a} T^{b}=\frac{n_{f}}{2} \delta^{a b}, \\
d_{A} & \equiv \delta^{a a}=N^{2}-1, \\
d_{F} & \equiv \delta_{i i}=d_{A} T_{F} / C_{F}=N n_{f},
\end{aligned}
$$

where the $T_{a}$ stand for the canonically normalized generators of $\mathrm{SU}(N)$ and the trace is taken over both color and flavor indices.

Our notation for the special functions that appear in the $\epsilon$-expansions of sum-integrals at finite density follows that of Ref. [8], which introduced

$$
\begin{aligned}
\zeta^{\prime}(x, y) & \equiv \partial_{x} \zeta(x, y) \\
\aleph(n, z) & \equiv \zeta^{\prime}(-n, z)+(-1)^{n+1} \zeta^{\prime}\left(-n, z^{*}\right), \\
\aleph(z) & \equiv \Psi(z)+\Psi\left(z^{*}\right) .
\end{aligned}
$$

Here, $\zeta$ stands for the generalized Riemann zeta function and $\Psi$ for the digamma function

$$
\Psi(w) \equiv \frac{\Gamma^{\prime}(w)}{\Gamma(w)}
$$

Finally, we will throughout the following chapters apply dimensional regularization at $d=4-2 \epsilon$ dimensions and (unless otherwise stated) in Euclidean metric, using as the momentum integration measure

$$
\begin{aligned}
\int_{p} \equiv \int \frac{d^{d-1} p}{(2 \pi)^{d-1}} & =\Lambda^{2 \epsilon} \int \frac{d^{d-1} p}{(2 \pi)^{d-1}} \equiv\left(\frac{e^{\gamma} \bar{\Lambda}^{2}}{4 \pi}\right)^{\epsilon} \int \frac{d^{d-1} p}{(2 \pi)^{d-1}} \\
\oiint_{P /\{P\}} & \equiv T \sum_{p_{0} /\left\{p_{0}\right\}} \int_{p},
\end{aligned}
$$

where $\bar{\Lambda} \equiv\left(4 \pi e^{-\gamma}\right)^{1 / 2} \Lambda$ is the scale parameter of the $\overline{\mathrm{MS}}$ scheme. The symbol $P /\{P\}$ refers to bosonic and fermionic momenta, respectively, for which capital letters stand for four-momenta and 
lower-case ones for three-momenta. Using these definitions, bosonic and fermionic 1-loop master integrals are defined by

$$
\mathcal{I}_{n}^{m} \equiv \sum_{P} \frac{\left(p_{0}\right)^{m}}{\left(P^{2}\right)^{n}}, \quad \widetilde{\mathcal{I}}_{n}^{m} \equiv \sum_{\{P\}} \frac{\left(p_{0}\right)^{m}}{\left(P^{2}\right)^{n}} .
$$

The bosonic and fermionic distribution functions at temperature $T$ and chemical potential $\mu$ are denoted by

$$
\begin{aligned}
n_{b}(k) & =\frac{1}{e^{k / T}-1} \\
n_{f}(k) & =\frac{1}{2}\left(\frac{1}{e^{(k-\mu) / T}+1}+\frac{1}{e^{(k+\mu) / T}+1}\right) .
\end{aligned}
$$

\section{PREVIOUS METHODS}

Let us begin by reviewing the main results available for the QCD pressure through various previous perturbative calculations in order to summarize the current understanding of the behavior of the quantity in various regions of the $\mu-T$ plane. The methods to be covered include dimensional reduction which has been used to determine the pressure up to and including order $g^{6} \ln g$ at finite $T$ and $\mu$ [7, 8], the perturbative $T=0$ techniques of Freedman and McLerran [9], and the HDL resummations that have proven to be highly effective at temperatures non-zero but parametrically smaller than $\mu$ 10, 16].

Recently there has been some progress to include effects of nonzero quark masses in the dimensional reduction approach [18] as well as to the next-to-leading order pressure at zero temperature [19]. In the following we shall always restrict our attention to the case of massless quarks.

\section{A. Dimensional reduction}

In finite-temperature QCD, dimensional reduction is based on the observation that at sufficiently high temperatures one has the parametric scale hierarchy $m_{\text {mag }} \ll m_{\mathrm{D}} \ll \pi T$ between the magnetic mass $\sim g^{2} T$, the Debye mass $\sim g T$, and the smallest non-zero Matsubara frequency, respectively. One may then integrate out all the non-static degrees of freedom from the original theory (corresponding to the last of the above energy scales), leaving behind a three-dimensional effective theory for the static gluon fields. At leading order, this integration-out can be performed explicitly, but beyond that it becomes more convenient to start from the most generic possible three-dimensional Lagrangian that respects the correct symmetries and to determine the values of its parameters by matching a set of simple physical quantities to their values in the full theory. This leads to a Lagrangian for the effective theory, commonly dubbed electrostatic QCD (EQCD), of the (Euclidean) form [6, 20]

$$
\begin{aligned}
\mathcal{L}_{\mathrm{EQCD}} & =\frac{1}{2} \operatorname{Tr} F_{i j}^{2}+\operatorname{Tr}\left[\left(D_{i} A_{0}\right)^{2}\right]+m_{\mathrm{E}}^{2} \operatorname{Tr} A_{0}^{2} \\
& +\frac{i g^{3}}{3 \pi^{2}} \sum_{f} \mu_{f} \operatorname{Tr} A_{0}^{3}+\lambda_{\mathrm{E}}^{(1)}\left(\operatorname{Tr} A_{0}^{2}\right)^{2}+\lambda_{\mathrm{E}}^{(2)} \operatorname{Tr} A_{0}^{4}+\delta \mathcal{L}_{\mathrm{E}} .
\end{aligned}
$$

In the above Lagrangian, $m_{\mathrm{E}}$ agrees to leading order with the physical Debye mass $m_{\mathrm{D}}$,

$$
m_{\mathrm{D}}=g \sqrt{\frac{C_{A}+T_{F}}{3} T^{2}+T_{F} \frac{\mu^{2}}{\pi^{2}}}
$$


and the last term $\delta \mathcal{L}_{\mathrm{E}}$ corresponds to higher-dimensional operators that can be neglected for most practical purposes, as they contribute e.g. to the pressure starting only at $\mathcal{O}\left(g^{7}\right)$. The cubic, $C$-odd term [20, 21] in Eq. (2.1) is only present at finite chemical potentials and contributes to the pressure at order $g^{6} \ln g$, (but turns out to be responsible for the leading order result of the off-diagonal quark number susceptibilities at zero chemical potential 22] ). The fields have been rescaled from their four-dimensional values by $\sqrt{T}$ to have the canonical dimensionality at $d=3$, and the field strength and covariant derivative contain the three-dimensional gauge coupling $g_{\mathrm{E}} \equiv g \sqrt{T}+\mathcal{O}\left(g^{3}\right)$. The new theory should be able to describe the physics of the original one at distance scales of order $1 /(g T)$ and higher.

Assuming $g$ to be sufficiently small (i.e. $T$ sufficiently high), the above integrating-out procedure can be continued one step further by removing also the massive longitudinal gluon $A_{0}$ from the theory, thus producing an effective three-dimensional pure Yang-Mills theory for the $A_{i}$ fields. This theory, magnetostatic QCD (MQCD), is defined by the Lagrangian

$$
\mathcal{L}_{\mathrm{MQCD}}=\frac{1}{2} \operatorname{Tr} F_{i j}^{2},
$$

with the gauge coupling constant $g_{\mathrm{M}}=g_{\mathrm{E}}+\mathcal{O}\left(g_{\mathrm{E}}^{3} / m_{\mathrm{E}}\right)$ appearing in the field strength tensor. The perturbative expansion of the pressure of the full theory can then be given in the form

$$
p=p_{\mathrm{E}}+p_{\mathrm{M}}+p_{\mathrm{G}},
$$

where the first term corresponds to the coefficient of the unit operator of EQCD, not explicitly written in Eq. (2.1), and can be evaluated through a strict perturbative expansion (implying no resummations) of the full theory pressure. The second term, $p_{\mathrm{M}}$, represents the same thing for MQCD and can be computed through a diagrammatic expansion of the pressure of this theory, while the last piece, $p_{\mathrm{G}}$, corresponds to the (only) fundamentally non-perturbative contribution to the full theory pressure in the form of the pressure of MQCD. To order $g^{6} \ln g$ - the current state-of-the-art of the field - the three terms have been computed at $\mu=0$ in Ref. [] and at $\mu \neq 0$ in Ref. [8]. In Eq. [A1) of Appendix A we quote the result of Ref. [8] to order $g^{5}$ in a simplified case where equal chemical potentials have been assumed for all quark flavors.

Formally, the condition ensuring the validity of dimensional reduction is that the temperature is the largest fundamental energy scale in the problem, so that the above scale hierarchies are satisfied. In the limit of low temperatures $T \ll \mu$, the Debye mass becomes just $m_{\mathrm{D}}=\sqrt{N_{f} / 2} g \mu / \pi$ which shows that the identification of the static degrees of freedom as the only IR sensitive ones is justified as long as $T \ll g \mu$ or, formally, $T \sim g^{x} \mu$, with any $x<1$. Assuming this to be the case and defining $\tau \equiv \pi T /\left(g^{x} \mu\right)$, one can then extend the validity of the standard dimensional reduction results to the case of an arbitrary $x<1$ by simply inserting this value of the temperature,

$$
\pi T=\tau g^{x} \mu,
$$

into Eq. (3.13) of Ref. [8] and expanding the result as a power series in $g$ up to but not including order $g^{6} \mu^{4}$. The expansions of the $\aleph$ functions appearing in the coefficient of the $g^{4}$ term with an argument $\mu /(2 \pi T)$ can be performed with the aid of the results of the Appendix D of the same reference.

Here, we exhibit the result of the above procedure only for the case of $x=1 / 2$, and as above we assume all quark flavors to have the same chemical potential ${ }^{2} \mu$, where we can use the simplified

\footnotetext{
${ }^{2}$ Here, one cannot simply multiply the result by $\frac{1}{N_{f}} \sum_{f}$ in order to generalize it to an arbitrary number of flavors with independent chemical potentials.
} 
result of Eq. (A1). After some straightforward manipulations we obtain

$$
\begin{aligned}
p_{\mathrm{DR}} & =\frac{d_{A} \mu^{4}}{(4 \pi)^{2}}\left\{\frac{4 d_{F}}{3 d_{A}}+g \frac{8 \tau^{2} d_{F}}{3 d_{A}}-g^{2}\left(\frac{T_{F}}{2 \pi^{2}}-\frac{16 \tau^{4}}{45}\left(1+\frac{7 d_{F}}{4 d_{A}}\right)\right)\right. \\
& -g^{3} \frac{T_{F} \tau^{2}}{\pi^{2}}+g^{7 / 2} \frac{4 T_{F}^{3 / 2} \tau}{3 \pi^{3}} \\
& -\frac{g^{4}}{576 \pi^{4}}\left(72 T_{F}^{2} \ln g+32\left(2 C_{A}+5 T_{F}\right) \pi^{2} \tau^{4}+4 C_{A} T_{F}\left(71-33 \ln 2+33 \ln \frac{\bar{\Lambda}}{\mu}\right)\right. \\
& \left.-153 C_{F} T_{F}+4 T_{F}^{2}\left(11-36 \gamma+36 \ln 8 \tau-12 \ln \frac{\bar{\Lambda}}{\mu}\right)\right) \\
& +g^{9 / 2} \frac{2 \sqrt{T_{F}}\left(C_{A}+T_{F}\right) \tau^{3}}{3 \pi^{2}} \\
& +\frac{g^{5} \tau^{2} T_{F}}{288 \pi^{4}}\left(4\left(53 C_{A}-6 C_{F}+2 T_{F}\right) \ln g+3 C_{F}\left(35+16 \frac{\zeta^{\prime}(-1)}{\zeta(-1)}-16 \ln 2 \tau\right)\right. \\
& +C_{A}\left(-227-20 \ln 2+48 \gamma+104 \frac{\zeta^{\prime}(-1)}{\zeta(-1)}+144 \ln \frac{T_{F}}{\pi^{2}}-152 \ln \tau-132 \ln \frac{\bar{\Lambda}}{\mu}\right) \\
& \left.-4 T_{F}\left(5+8 \ln 2-12 \gamma+16 \frac{\zeta^{\prime}(-1)}{\zeta(-1)}-12 \gamma-4 \ln \tau-12 \ln \frac{\bar{\Lambda}}{\mu}\right)\right) \\
& +\frac{g^{11 / 2} \tau}{72 \sqrt{T_{F}} \pi^{5}}\left(-33 C_{A} T_{F}^{2} \ln g+4 C_{A}^{2} \pi^{2} \tau^{4}+8 C_{A} T_{F} \pi^{2} \tau^{4}+4 T_{F}^{2} \pi^{2} \tau^{4}-54 C_{F} T_{F}^{2}\right. \\
& \left.+33 C_{A} T_{F}^{2}\left(1+2 \gamma-2 \ln 4 \tau+2 \ln \frac{\bar{\Lambda}}{\mu}\right)+12 T_{F}^{3}\left(1+2 \ln 2-2 \ln \frac{\bar{\Lambda}}{\mu}\right)\right) \\
& \left.+\frac{g^{6} \ln g \tau^{4}}{864 \pi^{4}}\left(166 C_{A}^{2}+\frac{832}{5} C_{A} T_{F}+72 C_{F} T_{F}+\frac{371}{5} T_{F}^{2}\right)+O\left(g^{6}\right)\right\} .
\end{aligned}
$$

To test the validity of this expression, one may compare it with the prediction of other approaches entirely independent of dimensional reduction which we will do in Sec. IV]

\section{B. The Freedman-McLerran result for the $T=0$ pressure}

At exactly zero temperature, important simplifications take place which resulted in the perturbative expansion of the QCD pressure being extended to order $g^{4}$ in this region already remarkably early, in the late 1970's 9]. The most important effects stem from the fact that at $T=0$ loop integrations become much more straightforward than at finite temperature due to the Matsubara sums reducing to ordinary integrals. The computation of Ref. [9] was organized in much the same way as the one to be presented in this paper, with the exception that most of the technical details were much less involved. Especially with zero quark masses, the evaluation of the 2PI graphs at zero temperature is significantly simpler than at $T \neq 0$, leaving as the real challenge the computation of the 'plasmon' sum, i.e. the resummation of ring diagrams. This they did in a highly imaginative - and to a large extent analytic — way, having to resort to numerics only in evaluating a few low-dimensional integrals.

While the original calculation of Ref. [9] was performed in the momentum subtraction scheme and moreover using by now somewhat out-dated methods that led to largish numerical error bars, the result was to a large extent independently ${ }^{3}$ rederived in Ref. [8] in the $\overline{\mathrm{MS}}$ scheme. For $N_{f}$

\footnotetext{
${ }^{3}$ With the exception of the plasmon sum, for which similar methods as those in Ref. [9] were used. A translation
} 
flavors of quarks at a equal chemical potential $\mu$, the result reads

$$
\begin{aligned}
p(\mu, T=0) & =\frac{d_{A} \mu^{4}}{2 \pi^{2}}\left(\frac{d_{F}}{6 d_{A}}-\frac{g^{2}}{(4 \pi)^{2}} T_{F}-\frac{g^{4}}{(4 \pi)^{4}} T_{F}\left[4 T_{F} \ln \frac{g^{2} T_{F}}{(4 \pi)^{2}}+\frac{2}{3}\left(11 C_{A}-4 T_{F}\right) \ln \frac{\bar{\Lambda}}{\mu}\right.\right. \\
& -\frac{17}{2} C_{F}+\frac{C_{A}}{36}(568-264 \ln 2) \\
& \left.\left.-2 T_{F}\left(\frac{88}{9}-14 \ln 2+\frac{16}{3}(\ln 2)^{2}-\delta-\frac{2 \pi^{2}}{3}\right)\right]+O\left(g^{6} \ln g\right)\right)
\end{aligned}
$$

where $\delta \approx-0.856383209 \ldots$ is defined through a one-dimensional integral in Ref. [8].

\section{Minimal HTL/HDL resummation}

At low but non-vanishing temperatures, the Freedman-McLerran result for the zero-temperature interaction pressure (where the Stefan-Boltzmann (SB) result has been subtracted off) to order $g^{4}$ needs to be corrected by $T$-dependent contributions. At two-loop order there are infrared safe terms of order $g^{2} \mu^{2} T^{2}$ which clearly are more important than the unknown four-loop $T=0$ terms $\sim g^{6} \mu^{4}$ as long as $T \gtrsim g^{2} \mu$. On the other hand, when $T$ approaches the scale $g \mu$ from the dimensional reduction region above, the leading contribution of ring diagrams $T m_{\mathrm{D}}^{3} \sim g^{3} T \mu^{3}$ becomes comparable to $g^{2} \mu^{2} T^{2}$. A naive extrapolation of the $T m_{\mathrm{D}}^{3}$ term to temperatures parametrically smaller than $g \mu$ would even suggest that for $T \sim g^{x} \mu$ with $x>1$ these become more important than the two-loop terms, as $T m_{\mathrm{D}}^{3} \sim g^{3+x} \mu \gg g^{2+2 x} \mu \sim g^{2} \mu^{2} T^{2}$. As we shall see, the $T$-dependent contributions from ring diagrams indeed become more important than the 2-loop term $g^{2} \mu^{2} T^{2}$ here, though they are not enhanced by a relative factor $g^{-(x-1)}$ as suggested by dimensional reduction, but instead only by a logarithm.

For temperatures $T \lesssim g \mu$, where dimensional reduction is no longer applicable, it becomes important to keep the nonstatic parts of the gluon self energy in the ring diagrams. At low momenta and frequencies of the order $g \mu$, the leading terms in the gluon self-energy are given by the so-called hard thermal loops (HTL) approximation with the overall $m_{\mathrm{D}}^{2}$ factor replaced by its zero-temperature value - a special case occasionally referred to as hard dense loops (HDL). In the longitudinal gluon propagator, one can observe the usual Debye screening effect at the frequency $\omega \ll g \mu$, but in the transverse propagator the situation is more complicated. At strictly zero frequency the magnetostatic HDL propagator is massless, but for small but nonvanishing frequencies $\omega \ll q \lesssim m_{\mathrm{D}}$ its inverse has the form

$$
q^{2}-\omega^{2}+\Pi_{\mathrm{T}}^{\mathrm{HDL}}(\omega, q)=q^{2}-\frac{i \pi m_{\mathrm{D}}^{2}}{4} \frac{\omega}{q}+O\left(\omega^{2}\right) .
$$

The transverse part of the propagator thus has a pole at imaginary $q$ and $|q|=m_{\text {mag }}(\omega)$, introducing a new parametrically small dynamical screening mass [31, 45]

$$
m_{\mathrm{mag}}(\omega)=\left(\frac{\pi m_{\mathrm{D}}^{2} \omega}{4}\right)^{1 / 3}, \quad \omega \ll m_{\mathrm{D}},
$$

which represents an in-medium version of Lenz's law. As soon as the temperature is small but nonvanishing, the ring diagrams obtain contributions involving the Bose-Einstein distribution function which leads to sensitivity to this additional scale. In these contributions, we effectively have

of the original result of Ref. [9] to the $\overline{\mathrm{MS}}$ scheme was previously given in the last reference of Ref. [26] and in Ref. [27]. 
$m_{\text {mag }}(\omega \sim T) \sim g^{(2+x) / 3} \mu$ for $T \sim g^{x} \mu$ and $x>1$. Note that this is parametrically smaller than $m_{\mathrm{D}} \sim g \mu$, but always larger than the magnetic mass scale of MQCD, $m_{\text {mag }}(\omega=0)=g^{2} T \sim g^{2+x} \mu$.

The resummation of the nonstatic transverse gluon self-energy gives rise to terms nonanalytic in the temperature which to lowest order in a low-temperature expansion turn out to be of the order $g^{2} \mu^{2} T^{2} \ln T$. This gives rise to so-called anomalous or non-Fermi-liquid behavior in the entropy and specific heat at low $T$, because instead of the usual linear behavior in $T$ the entropy then has a $T \ln T$ term which is the hallmark of a breakdown of the Fermi-liquid picture (first discussed in the context of nonrelativistic QED by Norton, Holstein and Pincus [23]). Indeed, inspection of the dispersion laws of fermionic quasiparticles reveals that there is a logarithmic singularity in the group velocity at the therefore no longer sharply defined Fermi surface ${ }^{4}$.

For a long time, only the multiplicative coefficient of the $T \ln T$ term in the specific heat was known. It was only rather recently [10] that also the scale under the logarithm was determined together with the next order terms in the low-temperature $(T \ll g \mu)$ series which in addition involves fractional powers of $T$ due to the cubic root in Eq. (2.9). For the pressure, these "anomalous" $T$-dependent contributions are contained in an expression, which was first derived in Ref. [16] and which we shall label by $\mathrm{HDL}^{+}$,

$$
\begin{aligned}
\frac{1}{N_{g}} \delta p^{\mathrm{HDL}^{+}} & =-\frac{g^{2} T_{F}}{48 \pi^{2}} \mu^{2} T^{2}-\frac{1}{2 \pi^{3}} \int_{0}^{\infty} d q_{0} n_{b}\left(q_{0}\right) \int_{0}^{\infty} d q q^{2}\left[2 \operatorname{Im} \ln \left(\frac{q^{2}-q_{0}^{2}+\Pi_{\mathrm{T}}^{\mathrm{HDL}}}{q^{2}-q_{0}^{2}}\right)\right. \\
& \left.+\operatorname{Im} \ln \left(\frac{q^{2}-q_{0}^{2}+\Pi_{\mathrm{L}}^{\mathrm{HDL}}}{q^{2}-q_{0}^{2}}\right)\right]+O\left(g^{2} T^{4}\right)+O\left(g^{3} \mu T^{3}\right)+O\left(g^{4} \mu^{2} T^{2}\right),
\end{aligned}
$$

with $\delta p$ denoting the temperature-dependent part of the interaction pressure

$$
\begin{aligned}
\delta p & \equiv \Delta p-\left.\Delta p\right|_{T=0}, \\
\Delta p & \equiv p-p_{\mathrm{SB}} .
\end{aligned}
$$

The expression (2.10) can be viewed as a minimal ${ }^{5}$ resummation of HDL diagrams, where the HDL self-energies are only kept in the infrared sensitive part of the ring diagrams involving the distribution function $n_{b}$, while infrared safe two-loop contributions are treated in an unresummed form.

\section{T parametrically smaller than $m_{D}$}

With $g \ll 1$ and $x>1$ in $T \sim g^{x} \mu$, the temperature is parametrically smaller than the Debye mass $m_{\mathrm{D}} \sim g \mu$ and Eq. (2.10) contains the leading contributions to the temperature-dependent parts of the interaction pressure, which ignoring logarithms are of order $g^{2} \mu^{2} T^{2} \sim g^{2+2 x} \mu^{4}$, while the higher-order terms in Eq. (2.10) are at least of order $g^{4+2 x} \mu^{4}$. The Freedman-McLerran result for the $T=0$ pressure, Eq. (2.7), is accurate to order $g^{4} \mu^{2}$ and its error is of order $g^{6} \mu^{4}$ (again ignoring logarithms of $g$ ). Eq. (2.10) thus represents the leading correction to the FreedmanMcLerran result as long as $x<2$ (i.e., $T \gtrsim g^{2} \mu$ ), whereas in quantities such as the entropy density

\footnotetext{
4 A systematic calculation of the group velocity beyond the leading-log approximation has only recently been carried out in Ref. [24].

${ }^{5}$ As opposed to the HTL/HDL resummation considered in [25, 26] which aims at improving the convergence of the perturbative series at high temperature by retaining higher-order effects from HTL/HDL physics beyond what is needed from a perturbative point of view. The + in $\mathrm{HDL}^{+}$and $\mathrm{HTL}^{+}$is meant as a reminder that the corresponding quantities are not expressed in terms of HTL/HDL quantities only, but combined with unresummed infrared-safe contributions.
} 
$s=\partial p / \partial T$ and the various specific heats, where the $T=0$ part of the pressure drops out, it is in fact the leading term in the interaction part for all $x \geq 1$.

In Eq. (2.10), $g$ appears only in the combination $\bar{g}^{2} \equiv g^{2} T_{F}$, and it is therefore convenient to define a reduced temperature variable

$$
\bar{\tau}=\pi T /\left(\bar{g}^{x} \mu\right) .
$$

For $x>1$, the perturbative content of Eq. (2.10) is that given by the low-temperature expansion worked out in Refs. [10, 16]. With the above variables, this reads

$$
\begin{aligned}
\frac{1}{N_{g}} \frac{\delta p^{\mathrm{HDL}^{+}}}{m_{\mathrm{D}}^{4}} & =\frac{\bar{\tau}^{2} \bar{g}^{2(x-1)}}{72}\left(\ln \left(\frac{1}{\bar{\tau} \bar{g}^{x-1}}\right)+\ln \frac{4}{\pi}+\gamma_{E}-\frac{6}{\pi^{2}} \zeta^{\prime}(2)-\frac{3}{2}\right) \\
& -\frac{2^{2 / 3} \Gamma\left(\frac{8}{3}\right) \zeta\left(\frac{8}{3}\right)}{3 \sqrt{3} \pi^{7 / 3}} \bar{\tau}^{8 / 3} \bar{g}^{8(x-1) / 3}+8 \frac{2^{1 / 3} \Gamma\left(\frac{10}{3}\right) \zeta\left(\frac{10}{3}\right)}{9 \sqrt{3} \pi^{11 / 3}} \bar{\tau}^{10 / 3} \bar{g}^{10(x-1) / 3} \\
& +\frac{2048-256 \pi^{2}-36 \pi^{4}+3 \pi^{6}}{2160 \pi^{2}} \bar{\tau}^{4} \bar{g}^{4(x-1)}\left[\ln \left(\frac{1}{\bar{\tau} \bar{g}^{x-1}}\right)+\ln \pi+\bar{c}\right] \\
& +O\left(\bar{g}^{14(x-1) / 3}\right)+O\left(g^{2 x}\right)
\end{aligned}
$$

where $\bar{c} \approx 4.0993485 \ldots$ is given by a numerical integral defined in Ref. [16]. The latter of the error terms in Eq. (2.13) corresponds to the leading-order terms to be expected from three- and higher-loop contributions ${ }^{6}$ proportional to $g^{4} \mu^{2} T^{2}$ which are presumably enhanced by logarithms of $T$ and $g$. Depending on the value of $x>1$, a finite number of terms in the low- $T$ expansion remain more important than this (see Fig. 17 in Sec. (V).

When $x=1$, i.e. $T \sim g \mu$, the expansion of Eq. (2.13) clearly breaks down (unless $\bar{\tau} \ll 1$ ) and the HDL-resummed expression of Eq. (2.10) therefore needs to be evaluated numerically as in Ref. 16]. This expression has then the form of $g^{4} \mu^{4}$ times a function of $T /(g \mu)$, and is therefore of the same order as the $g^{4}$ term of the $T=0$ pressure of Freedman and McLerran, to which it is to be added. As displayed in Ref. [16] for the case of the entropy, and as we shall see for the pressure in the plots of Section [V] of the present paper, the $T$-dependent terms of Eq. (2.13) smoothly interpolate between a dominant $g^{2} T^{2} \mu^{2} \ln T$ behavior at low temperature and the terms of order $g^{2} T^{2} \mu^{2}, g^{3} \mu^{3} T$, and $g^{4} \mu^{4} \ln T$ of the dimensional reduction pressure which should be the dominant terms at sufficiently high temperatures and which remain comparable to $g^{4} \mu^{4}$ as long as the parametric equality $T \sim g \mu$ holds.

\section{2. $T$ parametrically larger than $m_{D}$}

When $x<1$ in $T \sim g^{x} \mu$, i.e. $T \gg g \mu$, dimensional reduction provides the most accurate result available for the QCD pressure. Up to an error of the order of three-loop contributions proportional to $g^{4} \mu^{2} T^{2} \sim g^{4+2 x} \mu^{4}$, one can however reproduce its prediction by extending the above HDL-resummed calculation to include the leading thermal corrections to the gluon self-energy. In practice, this means replacing the HDL approximation by the HTL one and also keeping the order $g^{2} T^{4}$ terms originating from infrared-safe two-loop contributions to the pressure that were omitted in Eq. (2.10) because they were of too high order when $x \geq 1$. This possibility was mentioned in Ref. [16], but not considered further because that work concentrated on the region of $T \lesssim g \mu$. For the purposes of the present paper, we however write down the straightforward extension of

\footnotetext{
${ }^{6}$ There, $g^{2}$ no longer appears exclusively in combination with $T_{F}$.
} 
Eq. (2.10) to the HTL approximation in the form

$$
\begin{aligned}
\frac{1}{N_{g}} \delta p^{\mathrm{HDL}^{+}}= & -\frac{g^{2} T_{F}}{48 \pi^{2}} \mu^{2} T^{2}+\frac{g^{2}\left(2 C_{A}-T_{F}\right)}{288} T^{4} \\
& -\frac{1}{2 \pi^{3}} \int_{0}^{\infty} d q_{0} n_{b}\left(q_{0}\right) \int_{0}^{\infty} d q q^{2}\left[2 \operatorname{Im} \ln \left(\frac{q^{2}-q_{0}^{2}+\Pi_{\mathrm{T}}^{\mathrm{HTL}}}{q^{2}-q_{0}^{2}}\right)\right. \\
& \left.+\operatorname{Im} \ln \left(\frac{q^{2}-q_{0}^{2}+\Pi_{\mathrm{L}}^{\mathrm{HTL}}}{q^{2}-q_{0}^{2}}\right)\right]+O\left(g^{4} \mu^{2} T^{2}\right) .
\end{aligned}
$$

Combining the above expression with the Freedman-McLerran result of Eq. (2.7) to obtain

$$
\Delta p^{\mathrm{HDL}^{+}} \equiv p^{\mathrm{HDL}^{+}}-p_{\mathrm{SB}} \equiv \Delta p^{\mathrm{FMcL}}+\delta p^{\mathrm{HDL}^{+}},
$$

we have an expression for the interaction pressure whose error is of order $g^{\min (4+2 x, 6)}$ for all $T \sim g^{x} \mu$. This we shall compare (and thus test) in the following with our new approach which resums the complete one-loop gluon self-energy (i.e., not only the leading HTL/HDL contribution) in ring diagrams. Note that the accuracy of (2.15) is at least of order $g^{4}$ for all parametrically small temperatures, excluding only the case of $x=0$, where $T \sim \mu$.

\section{THE NEW APPROACH}

In this Section, we introduce our novel and strictly four-dimensional calculational scheme designed to reproduce the perturbative expansion of the QCD pressure up to and including order $g^{4}$ at all values of $\mu$ and $T$. Our guiding principle is that when faced with the necessity to sum up graphs with multiple self energy insertions to circumvent infrared problems, we consider the entire self energy and not only those parts which are identified as relevant in some effective field theory description, such as the Debye mass in dimensional reduction or the HTL/HDL self energy in the corresponding resummation schemes. Because we (at present) limit ourselves to order $g^{4}$ accuracy, it will be sufficient to resum only one-loop self-energies in the infrared sensitive graphs, while IR safe diagrams will be treated perturbatively, using bare propagators. This will introduce gauge dependence to our results, but only at orders beyond $g^{4}$ which we will explicitly discard by either considering values of $g$ low enough for the higher order terms to be negligible or by performing numerical series expansions up to $\mathcal{O}\left(g^{4}\right)$.

We begin our treatment with a general diagrammatic analysis where we identify the relevant classes of Feynman graphs that need to be considered. After that, we describe their evaluation and show how adding them together leads to the final result displayed in Section IIIE Many details of the calculations as well as the results of several individual pieces of the result are left to be covered in the Appendices.

\section{A. Identification of the relevant diagrams}

To determine the QCD pressure up to and including order $g^{4}$ on the entire deconfined phase diagram of the theory, our first task is to identify all Feynman diagrams that contribute to the partition function at this order. These trivially include the two-gluon-irreducible (2GI) diagrams up to three-loop order, displayed in Fig. 1 which a straightforward power counting as well as the explicit calculation of Ref. [8] confirms as infrared finite for all temperatures and chemical potentials.

In addition to these cases, there are, however, several other classes of IR sensitive diagrams that need to be resummed to infinite loop order, as a power counting reveals that the dressing 
a)<smiles>C1CCCCCC1</smiles>

d)

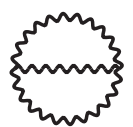

h)

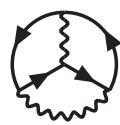

m)

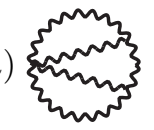

b)<smiles>C1CCCCCC1</smiles>

e)

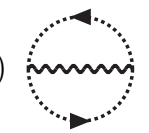

i)

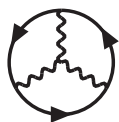

n)

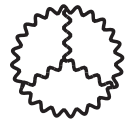

c)

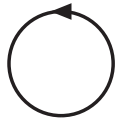

f)

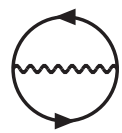

j)
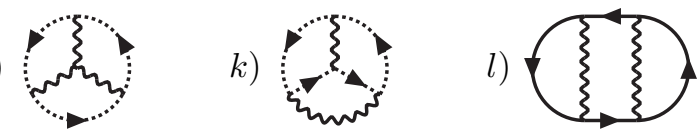

o)

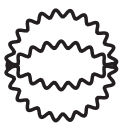

k)

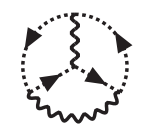

g)

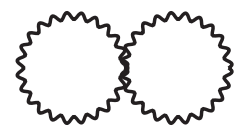

p)

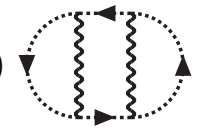

FIG. 1: The one-, two- and three-loop two-gluon-irreducible (2GI) graphs of QCD. The wavy line stands for a gluon, the dotted line a ghost and the solid line a quark.

of (at least some of) their gluon lines with an arbitrary number of one loop gluon polarization tensors does not increase their order beyond $g^{4}$. These diagrams are shown in Fig. 2, where the first set corresponds to the well-known class of ring diagrams that leads to the known $g^{3}$ and $g^{4} \ln g$ contributions to the pressure at high $T$ [2, 3] and to the $g^{4} \ln g$ term at $T=0$ [9]. Among others, this class contains the set of all three-loop two particle reducible (2PR) graphs of the theory which are missing from Fig. 1.

As we shall see (in contradiction to the opposite assertion in Ref. [2]), the resummation of the ring diagrams is, however, not enough to obtain the entire order $g^{4}$ term correctly at nonzero $T$. Although without resummation starting at orders $g^{6}, g^{8}$ and $g^{6}$, respectively, the classes of Fig. 2 b$\mathrm{d}$, corresponding to self-energy insertions in the gluonic two-loop 2GI diagrams $1 \mathrm{~d}$ and $1 \mathrm{~g}$, have the potential to give rise to contributions of order $\mathcal{O}\left(g^{4} T^{2} \mu^{2}\right)$ and $\mathcal{O}\left(g^{4} T^{4}\right)$ to the pressure. When $T$ is not parametrically larger than $m_{\mathrm{D}}$, it turns out that only the class b gives a non-zero contribution at this order, being proportional to $g^{2} T^{2} m_{\mathrm{D}}^{2}$. When $T \sim g^{x} \mu$ with $x>0$, none of the three classes contributes to the pressure to order $g^{4} \mu^{4}$, but in the calculation of the low-temperature entropy and specific heat they have to be taken into account already at order $g^{4} \mu^{2} T$.

For any other classes of diagrams apart from those shown in Figs. 1 and 2, it is very straightforward to see that the contributions will be beyond order $g^{4}$. In particular, if we were to add an additional gluon line with some number of self energy insertions into the graphs of Fig. 2 b-d (i.e. dressing the three-loop 2GI diagrams with self energies), we would notice that the two extra insertions of the coupling constant due to the new vertices (vertex) ensure that these graphs only contribute to the pressure at order $g^{6}$. Similarly, one can see that the inclusion of the two-loop self energy into the ring diagrams only has an effect on the pressure starting at $\mathcal{O}\left(g^{5}\right)$.

\section{B. The 2GI diagrams}

In Feynman gauge, the sum of the 2GI diagrams in Fig. 1 at arbitrary $T$ and $\mu$ can be directly extracted from from Ref. [8] with the result

$$
p_{2 \mathrm{GI}}=\pi^{2} d_{A} T^{4}\left(\frac{1}{45}\left\{1+\frac{d_{F}}{d_{A}}\left(\frac{7}{4}+30 \bar{\mu}^{2}+60 \bar{\mu}^{4}\right)\right\}\right.
$$




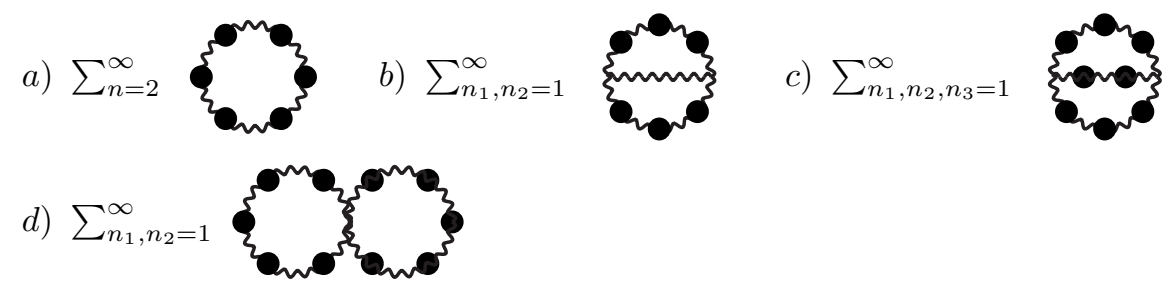

FIG. 2: Classes of IR sensitive vacuum graphs contributing to the QCD pressure at order $g^{4}$. The black dots represent the one-loop gluon polarization tensor given in Fig. 3a and the indices $n_{i}$ stand for the numbers of loop insertions on the respective lines.

$$
\begin{aligned}
& -\frac{g^{2}}{9(4 \pi)^{2}}\left\{C_{A}+\frac{T_{F}}{2}\left(1+12 \bar{\mu}^{2}\right)\left(5+12 \bar{\mu}^{2}\right)\right\} \\
& -\frac{g^{4}}{54(4 \pi)^{4}}\left\{\frac{23 C_{A}^{2}-C_{A} T_{F}\left(29+360 \bar{\mu}^{2}+720 \bar{\mu}^{4}\right)+4 T_{F}^{2}\left(5+72 \bar{\mu}^{2}+144 \bar{\mu}^{4}\right)}{\epsilon}\right. \\
& +C_{A}^{2}\left(182 \ln \frac{\bar{\Lambda}}{4 \pi T}+247+272 \frac{\zeta^{\prime}(-1)}{\zeta(-1)}-90 \frac{\zeta^{\prime}(-3)}{\zeta(-3)}\right) \\
& +C_{A} T_{F}\left(-16\left(5+36 \bar{\mu}^{2}+72 \bar{\mu}^{4}\right) \ln \frac{\bar{\Lambda}}{4 \pi T}-\frac{217}{5}-56 \frac{\zeta^{\prime}(-1)}{\zeta(-1)}+\frac{72}{5} \frac{\zeta^{\prime}(-3)}{\zeta(-3)}\right. \\
& \left.+24\left(9+4 \frac{\zeta^{\prime}(-1)}{\zeta(-1)}\right) \bar{\mu}^{2}+432 \bar{\mu}^{4}+144\left(1+4 \bar{\mu}^{2}\right) \aleph(1, z)+3456 \aleph(3, z)\right) \\
& +4 T_{F}^{2}\left(\left(1+12 \bar{\mu}^{2}\right)\left(4\left(5+12 \bar{\mu}^{2}\right) \ln \frac{\bar{\Lambda}}{4 \pi T}+15+8 \frac{\zeta^{\prime}(-1)}{\zeta(-1)}+36 \bar{\mu}^{2}\right)\right. \\
& \left.+144\left(1+4 \bar{\mu}^{2}\right) \aleph(1, z)\right)-9 C_{F} T_{F}\left(\frac{35}{2}-16 \frac{\zeta^{\prime}(-1)}{\zeta(-1)}+4\left(59+16 \frac{\zeta^{\prime}(-1)}{\zeta(-1)}\right) \bar{\mu}^{2}\right. \\
& \left.\left.\left.+664 \bar{\mu}^{4}+96\left(i \bar{\mu}\left(1+4 \bar{\mu}^{2}\right) \aleph(0, z)+2\left(1+8 \bar{\mu}^{2}\right) \aleph(1, z)-12 i \bar{\mu} \aleph(2, z)\right)\right)\right\}\right),
\end{aligned}
$$

where $\bar{\mu} \equiv \mu /(2 \pi T)$ and where we have renormalized the gauge coupling using the usual zerotemperature renormalization constant $Z_{g}$. The sum, however, still contains uncanceled UV $1 / \epsilon$ divergences and depends on the choice of gauge, so that it has no separate physical significance.

\section{The ring sum}

To order $g^{4}$, the ring sum of Fig. 2 a can be separated into three pieces $p_{\mathrm{Vv}}, p_{\mathrm{VM}}$ and $p_{\text {ring }}$ according to Fig. 3 by decomposing the one-loop gluon polarization tensor (see Appendix C) into its vacuum $(T=\mu=0)$ and matter parts. Note that only the matter part has to be resummed, as the vacuum parts contribute to order $g^{4}$ only through the two three-loop diagrams in Figs. 3b and c. ${ }^{7}$ The evaluation of $p_{\mathrm{VV}}$ and $p_{\mathrm{VM}}$ is relatively straightforward, and fully analytic expressions for them are given in Appendix B 1

\footnotetext{
7 Take any graph $G$ belonging to the ring sum and having four or more loops and at least one vacuum tensor insertion, and consider it in the Feynman gauge. Applying Eq. C4 to it and contracting the Lorentz indices of the vacuum tensor with one of its neighboring gluon propagators, we see that $G$ is proportional to $g^{2}$ times a similar
} 


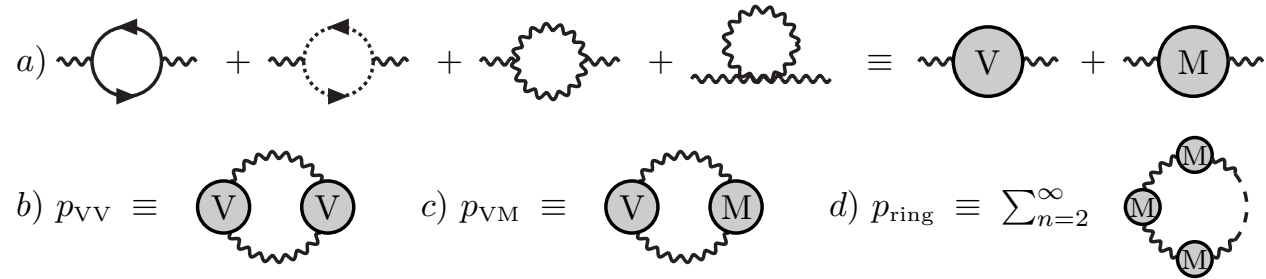

FIG. 3: a) The one-loop gluon polarization tensor $\Pi_{\mu \nu}(P)$ divided into its vacuum $(T=\mu=0)$ and matter (vacuum-subtracted) parts.

b) The IR-safe Vac-Vac diagram contributing to the pressure at $\mathcal{O}\left(g^{4}\right)$.

d) The IR-safe Vac-Mat diagram contributing to the pressure at $\mathcal{O}\left(g^{4}\right)$.

d) The remaining 'matter' ring sum.

To evaluate the remaining matter ring sum $p_{\text {ring }}$ we define the standard longitudinal and transverse parts of the vacuum-subtracted polarization tensor at $d=4-2 \epsilon$ by

$$
\begin{aligned}
\Pi_{\mathrm{L}}(P) \delta^{a b} & =\frac{P^{2}}{p^{2}}\left(\Pi_{00}^{a b}(P)-\left.\Pi_{00}^{a b}(P)\right|_{\mathrm{vac}}\right), \\
\Pi_{\mathrm{T}}(P) \delta^{a b} & =\frac{1}{d-2}\left(\Pi_{\mu \mu}^{a b}(P)-\left.\Pi_{\mu \mu}^{a b}(P)\right|_{\mathrm{vac}}-\frac{P^{2}}{p^{2}}\left(\Pi_{00}^{a b}(P)-\left.\Pi_{00}^{a b}(P)\right|_{\mathrm{vac}}\right)\right),
\end{aligned}
$$

where we have used the fact [36] that the one-loop gluon polarization tensor is transverse with respect to the four-momentum $P$ in the Feynman gauge. In terms of $\Pi_{\mathrm{T}}$ and $\Pi_{\mathrm{L}}$, the sum of the ring diagrams is then readily performed with the result

$$
\begin{aligned}
p_{\text {ring }} & =-\frac{d_{A}}{2} \sum_{P}\left\{\ln \left[1+\Pi_{\mathrm{L}}(P) / P^{2}\right]-\Pi_{\mathrm{L}}(P) / P^{2}\right. \\
& \left.+(d-2)\left(\ln \left[1+\Pi_{\mathrm{T}}(P) / P^{2}\right]-\Pi_{\mathrm{T}}(P) / P^{2}\right)\right\},
\end{aligned}
$$

which is now explicitly IR safe.

As the functions $\Pi_{\mathrm{L}}(P)$ and $\Pi_{\mathrm{T}}(P)$ behave at large $P^{2}$ like (see Sec. B.1.2 of Ref. [17])

$$
\Pi_{\mathrm{L} / \mathrm{T}}(P) \underset{P^{2} \rightarrow \infty}{\longrightarrow}-2(1+\epsilon) C_{A} g^{2} \sum_{Q} \frac{1}{Q^{2}}+\mathcal{O}\left(1 / P^{2}\right) \equiv \Pi_{\mathrm{UV}}+\mathcal{O}\left(1 / P^{2}\right),
$$

it is, however, immediately obvious that the sum-integral of Eq. (3.4) is still logarithmically divergent in the ultraviolet at $T \neq 0$. To regulate the divergence, we add and subtract a term of the form $(1+d-2)\left(\Pi_{\mathrm{UV}}\right)^{2} /\left(2\left(P^{2}+m^{2}\right)^{2}\right)$ from the integrand, with $m$ being an arbitrary mass parameter shielding it from IR divergences. By further adding and subtracting the corresponding massless term from the counterterm, we obtain three separate contributions to $p_{\text {ring }}$ : an UV and IR finite (at least to order $g^{4}$ - see below), $m$-dependent ring sum $p_{\text {ring }}^{\text {finite }}$, an UV finite, but IR divergent and $m$-dependent $p_{\text {ring }}^{\mathrm{IR}}$ and an UV and IR divergent and massless $p_{\text {ring }}^{\mathrm{UV}}$

$$
p_{\text {ring }}=p_{\text {ring }}^{\mathrm{finite}}+p_{\text {ring }}^{\mathrm{IR}}+p_{\text {ring }}^{\mathrm{UV}},
$$

graph with the vacuum insertion removed. But this graph is nothing but one of those diagrams that appeared in the original sum which implies that $G$ has to be proportional to at least the fifth power of the coupling. 


$$
\begin{aligned}
p_{\text {ring }}^{\text {finite }} & =-\frac{d_{A}}{2} \sum_{P}\left\{\ln \left[1+\Pi_{\mathrm{L}}(P) / P^{2}\right]-\Pi_{\mathrm{L}}(P) / P^{2}+C_{A}^{2} g^{4} T^{4} /\left(72\left(P^{2}+m^{2}\right)^{2}\right)\right. \\
& \left.+2\left(\ln \left[1+\Pi_{\mathrm{T}}(P) / P^{2}\right]-\Pi_{\mathrm{T}}(P) / P^{2}+C_{A}^{2} g^{4} T^{4} /\left(72\left(P^{2}+m^{2}\right)^{2}\right)\right)\right\} \\
p_{\text {ring }}^{\mathrm{IR}} & \equiv \frac{d_{A} C_{A}^{2} g^{4} T^{4}}{48} \sum_{P}\left\{\frac{1}{\left(P^{2}+m^{2}\right)^{2}}-\frac{1}{P^{4}}\right\} \\
p_{\text {ring }}^{\mathrm{UV}} & =\frac{1}{4}(d-1) d_{A}\left(\Pi_{\mathrm{UV}}\right)^{2} \sum_{P} \frac{1}{P^{4}} .
\end{aligned}
$$

The two first terms can be evaluated numerically at $\epsilon=0$ while the third one needs to be regulated with finite $\epsilon$. It is noteworthy that one can set $\epsilon=0$ even in the formally divergent $p_{\text {ring }}^{\mathrm{IR}}$ due to the fact that its IR divergence originates solely from the zeroth Matsubara mode of its second term which vanishes identically in dimensional regularization. The explicit values of $p_{\text {ring }}^{\mathrm{IR}}$ and $p_{\text {ring }}^{\mathrm{UV}}$ are given in Appendix B2 while the numerical evaluation of $p_{\text {ring }}^{\text {finite }}$ is the subject of Appendix D.

\section{The double and triple sums}

If the sums in Figs. $2 \mathrm{~b}-\mathrm{d}$ were to start from $n=0$, these multiple resummations would clearly correspond to the dressing of the propagators in three two-loop diagrams with the one-loop gluon polarization tensor. In the present case, we instead define a four-dimensionally transverse ${ }^{8}$ tensor $\Delta G_{\mu \nu}(P)$ by the equations

$$
\begin{aligned}
& \Delta G_{\mathrm{L}}(P)=\frac{1}{P^{2}+\Pi_{\mathrm{L}}(P)}-\frac{1}{P^{2}}=-\frac{\Pi_{\mathrm{L}}(P)}{P^{2}\left(P^{2}+\Pi_{\mathrm{L}}(P)\right)}, \\
& \Delta G_{\mathrm{T}}(P)=\frac{1}{P^{2}+\Pi_{\mathrm{T}}(P)}-\frac{1}{P^{2}}=-\frac{\Pi_{\mathrm{T}}(P)}{P^{2}\left(P^{2}+\Pi_{\mathrm{T}}(P)\right)},
\end{aligned}
$$

corresponding to the difference of a dressed (with the vacuum-subtracted self energy) and a bare gluon propagator in the Feynman gauge. It is a straightforward exercise in combinatorics to show that the symmetry factors of all graphs in Figs. 2b-d equal $1 / 4$ independently of $n$ - a result particularly obvious in $2 \mathrm{PI}$ formalism. To order $g^{4}$, these three classes of diagrams, denoted here by $p_{\mathrm{b}}, p_{\mathrm{c}}$ and $p_{\mathrm{d}}$, can then be written in the forms

$$
\begin{aligned}
p_{\mathrm{b}} & =\frac{d_{A} C_{A}}{4} g^{2} \sum_{P Q} \frac{\Delta G_{\mu \mu^{\prime}}(P) \Delta G_{\rho \rho^{\prime}}(Q)}{(P+Q)^{2}} \\
& \times\left(g^{\mu \nu}(2 P+Q)^{\rho}-g^{\nu \rho}(2 Q+P)^{\mu}+g^{\rho \mu}(Q-P)^{\nu}\right) \\
& \times\left(g^{\mu^{\prime} \nu}(2 P+Q)^{\rho^{\prime}}-g^{\nu \rho^{\prime}}(2 Q+P)^{\mu^{\prime}}+g^{\rho^{\prime} \mu^{\prime}}(Q-P)^{\nu}\right)+\mathcal{O}\left(g^{6}\right), \\
p_{\mathrm{c}} & =\frac{d_{A} C_{A}}{12} g^{2} \oint_{P Q} \Delta G_{\mu \mu^{\prime}}(P) \Delta G_{\rho \rho^{\prime}}(Q) \Delta G_{\nu \nu^{\prime}}(P+Q) \\
& \times\left(g^{\mu \nu}(2 P+Q)^{\rho}-g^{\nu \rho}(2 Q+P)^{\mu}+g^{\rho \mu}(Q-P)^{\nu}\right) \\
& \times\left(g^{\mu^{\prime} \nu^{\prime}}(2 P+Q)^{\rho^{\prime}}-g^{\nu^{\prime} \rho^{\prime}}(2 Q+P)^{\mu^{\prime}}+g^{\rho^{\prime} \mu^{\prime}}(Q-P)^{\nu^{\prime}}\right)+\mathcal{O}\left(g^{6}\right), \\
p_{\mathrm{d}} & =-\frac{d_{A} C_{A}}{2} g^{2} \sum_{P Q}\left(\Delta G_{\mu \mu}(P) \Delta G_{\nu \nu}(Q)-\Delta G_{\mu \nu}(P) \Delta G_{\mu \nu}(Q)\right)+\mathcal{O}\left(g^{6}\right) .
\end{aligned}
$$

\footnotetext{
${ }^{8}$ Thus decomposable into three-dimensionally transverse and longitudinal parts.
} 
All contributions involving the vacuum piece of the polarization tensor have been discarded as being of order $g^{6}$, following a reasoning similar to that in Footnote 7.

It is worthwhile to first perform a power counting analysis to determine at which order the above sum-integrals start to contribute to the pressure. In the regime of dimensional reduction, where $T \sim g^{x} \mu$ with $x<1$, one merely needs to consider the contributions of the zeroth Matsubara modes, as for the others the temperature acts as an infrared cutoff, leading to their values being proportional to at least $g^{5} T \mu^{3} \sim g^{5+x} \mu^{4}$. In the region $T \sim g \mu$, the Debye mass is, however, of the same order as the temperature, implying that all Matsubara modes give contributions to the pressure parametrically similar in magnitude. Scaling the three momenta in the integrals of Eqs. (3.11) - 3.13) by $g \mu$, one quickly sees that the results for the sum-integrals in this regime can up to $\mathcal{O}\left(g^{4}\right)$ be written in the form $g^{4} T^{2} \mu^{2} f(T /(g \mu))$, where the contributions of the non-static modes to the function $f$ vanish as the parameter $T /(g \mu)$ approaches infinity, while in the opposite limit $T /(g \mu) \rightarrow 0$ the function approaches a constant. As long as we are interested in the value of the pressure only to order $g^{4} \mu^{4}$, these graphs can clearly be altogether ignored. They will become relevant in the determination of the $\mathcal{O}\left(g^{4} \mu^{2} T\right)$ contributions to the specific heats, but this is outside the scope of the present work.

For now, we can concentrate our attention to the regime of dimensional reduction and therefore to the zero Matsubara mode parts of the above sum-integrals. Here, we encounter an important simplification which results from the fact that only the longitudinal part of the static gluon polarization tensor has a non-zero zero momentum limit at one-loop order. As the finite momentum corrections to the functions $\Pi_{\mathrm{L}}(P)$ and $\Pi_{\mathrm{T}}(P)$ clearly correspond to higher perturbative orders, we can simply replace

$$
\Delta G_{\mu \nu}(P) \rightarrow-\frac{m_{\mathrm{D}}^{2}}{p^{2}\left(p^{2}+m_{\mathrm{D}}^{2}\right)} \delta_{\mu 0} \delta_{\nu 0}
$$

in the integrals, leading to a dramatic reduction: both $p_{\mathrm{c}}$ and $p_{\mathrm{d}}$ then vanish identically. This can, however, be easily understood from the point of view of the three-dimensional effective theory EQCD as a demonstration of the fact that the $A_{0}^{3}$ and $A_{0}^{4}$ operators in its Lagrangian are not accompanied by couplings of order $g$ and $g^{2}$, respectively, but only $g^{3}$ (at nonzero $\mu$ ) and $g^{4}$.

In contrast to the above, for $p_{\mathrm{b}}$ one does obtain a non-zero value which has a direct parallel in EQCD in the form of an $\mathcal{O}(\mathrm{g})$ coupling between one massless $A_{i}$ and two massive $A_{0}$ fields and a corresponding two-loop diagram with one $A_{i}$ and two $A_{0}$ lines. Applying the limit of Eq. (3.14) to the sum-integral of Eq. (3.11), it is easy to see that we can reduce the expression of $p_{\mathrm{b}}$ (to order $\left.g^{4}\right)$ to the simple form

$$
p_{\mathrm{b}}=\frac{d_{A} C_{A}}{4} T^{2} m_{\mathrm{D}}^{4} g^{2} \int \frac{d^{3} p}{(2 \pi)^{3}} \int \frac{d^{3} q}{(2 \pi)^{3}} \frac{(\mathbf{p}-\mathbf{q})^{2}}{\mathbf{p}^{2}\left(\mathbf{p}^{2}+m_{\mathrm{D}}^{2}\right) \mathbf{q}^{2}\left(\mathbf{q}^{2}+m_{\mathrm{D}}^{2}\right)(\mathbf{p}+\mathbf{q})^{2}}
$$

which can be solved straightforwardly by introducing three Feynman parameters and using standard formulae for one-loop integrals in three dimensions. After some work, we get

$$
\begin{aligned}
p_{\mathrm{b}} & =\frac{d_{A} C_{A}}{4} T^{2} m_{\mathrm{D}}^{4} g^{2}\left\{\int \frac{d^{3} p}{(2 \pi)^{3}} \frac{1}{\mathbf{p}^{2}\left(\mathbf{p}^{2}+m_{\mathrm{D}}^{2}\right)} \int_{0}^{1} d x \int \frac{d^{3} q}{(2 \pi)^{3}} \frac{1}{\mathbf{q}^{2}+2 x \mathbf{q} \cdot \mathbf{p}+x \mathbf{p}^{2}+(1-x) m_{\mathrm{D}}^{2}}\right. \\
& \left.-\left(\int_{0}^{1} d x \int \frac{d^{3} p}{(2 \pi)^{3}} \frac{1}{\left(\mathbf{p}^{2}+x m_{\mathrm{D}}^{2}\right)^{2}}\right)^{2}\right\} \\
& =\frac{d_{A} C_{A}}{4} \frac{T^{2} m_{\mathrm{D}}^{4} g^{2}}{\left(4 \pi m_{\mathrm{D}}\right)^{2}}\left\{\frac{1}{\pi} \int_{0}^{1} d x \frac{1}{\sqrt{x(1-x)}} \int_{0}^{1} d y \frac{1}{\sqrt{y}} \frac{1}{1-y+y / x} \int_{0}^{1} d z \frac{1}{\sqrt{z}}-1\right\} \\
& =-\frac{d_{A} C_{A}}{4} T^{2} m_{\mathrm{D}}^{2} \frac{g^{2}}{(4 \pi)^{2}}(1-4 \ln 2)
\end{aligned}
$$


which we identify as the entire contribution of the classes b-d of Fig. 2 to the QCD pressure up to order $g^{4}$.

\section{E. The result}

We are now ready to write down our final result for the pressure, valid on the entire deconfined phase of QCD and accurate up to and including order $g^{4}$. Assembling all the various pieces, this function reads

$$
\begin{aligned}
p & =\left(p_{2 \mathrm{GI}}+p_{\mathrm{VV}}+p_{\mathrm{VM}}+p_{\text {ring }}^{\mathrm{UV}}+p_{\mathrm{b}}\right)+\left(p_{\text {ring }}^{\mathrm{IR}}+p_{\text {ring }}^{\mathrm{finite}}\right)+\mathcal{O}\left(g^{5} T \mu^{3}\right)+\mathcal{O}\left(g^{6} \mu^{4}\right) \\
& \equiv p_{\text {anl }}+p_{\text {ring }}^{\text {safe }}+\mathcal{O}\left(g^{5} T \mu^{3}\right)+\mathcal{O}\left(g^{6} \mu^{4}\right),
\end{aligned}
$$

where $p_{\text {anl }}$ stands for the sum of the first five terms in Eq. (3.17) and

$$
p_{\text {ring }}^{\text {safe }} \equiv p_{\text {ring }}^{\text {finite }}+p_{\text {ring }}^{\mathrm{IR}}
$$

is to be evaluated numerically. One should note that in this notation all $m$-dependence in contained in the two pieces of $p_{\text {ring }}^{\text {safe }}$, naturally canceling in their sum. In addition, it is worthwhile to point out that the inclusion of the term $p_{b}$ in Eq. (3.17) is inconsistent in the region of $T \sim g^{x} \mu, x \geq 1$ where we have neglected several contributions of the same magnitude. As this term, however, is of order $g^{4+2 x} \mu^{4}$, i.e. at least of order $g^{6} \mu^{4}$ in the region in question, the inconsistency is in any case beyond the order to which our result is indicated to be valid and can therefore be ignored.

Collecting the expressions for all of its parts from above and from Appendix B, the function $p_{\text {anl }}$ reads

$$
\begin{aligned}
p_{\text {anl }} & =\pi^{2} d_{A} T^{4}\left(\frac{1}{45}\left\{1+\frac{d_{F}}{d_{A}}\left(\frac{7}{4}+30 \bar{\mu}^{2}+60 \bar{\mu}^{4}\right)\right\}\right. \\
& -\frac{g^{2}}{9(4 \pi)^{2}}\left\{C_{A}+\frac{T_{F}}{2}\left(1+12 \bar{\mu}^{2}\right)\left(5+12 \bar{\mu}^{2}\right)\right\} \\
& +\frac{g^{4}}{27(4 \pi)^{4}}\left\{-C_{A}^{2}\left(22 \ln \frac{\bar{\Lambda}}{4 \pi T}+63-18 \gamma+110 \frac{\zeta^{\prime}(-1)}{\zeta(-1)}-70 \frac{\zeta^{\prime}(-3)}{\zeta(-3)}\right)\right. \\
& -C_{A} T_{F}\left(\left(47+792 \bar{\mu}^{2}+1584 \bar{\mu}^{4}\right) \ln \frac{\bar{\Lambda}}{4 \pi T}+\frac{2391}{20}+4 \frac{\zeta^{\prime}(-1)}{\zeta(-1)}+\frac{116}{5} \frac{\zeta^{\prime}(-3)}{\zeta(-3)}\right. \\
& \left.+6\left(257+88 \frac{\zeta^{\prime}(-1)}{\zeta(-1)}\right) \bar{\mu}^{2}+2220 \bar{\mu}^{4}+792\left(1+4 \bar{\mu}^{2}\right) \aleph(1, z)+3168 \aleph(3, z)\right) \\
& +T_{F}^{2}\left(\left(1+12 \bar{\mu}^{2}\right)\left(4\left(5+12 \bar{\mu}^{2}\right) \ln \frac{\bar{\Lambda}}{4 \pi T}+16 \frac{\zeta^{\prime}(-1)}{\zeta(-1)}\right)+\frac{99}{5}+\frac{16}{5} \frac{\zeta^{\prime}(-3)}{\zeta(-3)}\right. \\
& \left.+312 \bar{\mu}^{2}+624 \bar{\mu}^{4}+288\left(1+4 \bar{\mu}^{2}\right) \aleph(1, z)+1152 \aleph(3, z)\right) \\
& +\frac{9}{4} C_{F} T_{F}\left(35-32\left(1-4 \bar{\mu}^{2}\right) \frac{\zeta^{\prime}(-1)}{\zeta(-1)}+472 \bar{\mu}^{2}+1328 \bar{\mu}^{4}\right. \\
& \left.\left.\left.+192\left(i \bar{\mu}\left(1+4 \bar{\mu}^{2}\right) \aleph(0, z)+2\left(1+8 \bar{\mu}^{2}\right) \aleph(1, z)-12 i \bar{\mu} \aleph(2, z)\right)\right)\right\}\right) .
\end{aligned}
$$

Not only have all the UV divergences canceled between the different parts of this result, once the renormalization of the gauge coupling $g$ has been taken care of, but this expression actually contains all the (explicit) renormalization scale dependence of the pressure up to the present order 
in perfect agreement with Ref. [8], leaving $p_{\text {ring }}^{\text {safe }}$ entirely independent of the parameter $\bar{\Lambda}$. Eq. (3.20) is also valid for all values of $T$ and $\mu$; the limit for $\mu \rightarrow 0$ is given in Eq. (B7) and the limit $T \rightarrow 0$ in Eq. (B8). All terms non-analytic in $g^{2}$ are contained in the piece $p_{\text {ring }}^{\text {safe }}$ awaiting numerical evaluation.

In the following we shall denote our final result for the pressure - which is accurate to order $g^{4}$ for all values of $T$ and $\mu$ (while also containing some incomplete contributions of higher order, to be discarded later) — by

$$
p_{\mathrm{IV}}=p_{\mathrm{anl}}+p_{\text {ring }}^{\mathrm{safe}} \text {. }
$$

\section{F. Numerical infrared issues}

Before moving on to examining our result by numerically evaluating the function $p_{r i n g}^{\text {finite }}$ in Eq. (3.6), there is one more practical issue related to the magnetic mass problem [28, 29] that needs to be dealt with. To wit, in the limit $P \rightarrow 0$, the argument of $\ln \left(1+\Pi_{\mathrm{T}}(P) / P^{2}\right)$ becomes negative, resulting in an unwanted imaginary contribution to the integral which actually renders

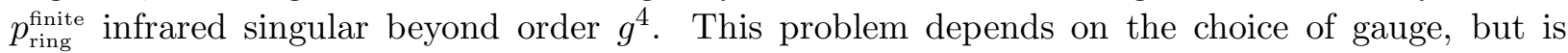
present in all covariant gauges (as well as the Coulomb gauge).

The origin of the problem can be traced back to the fact that when dressed with the full oneloop self-energy, the transverse part of the gluon propagator develops a space-like pole. For $p_{0}=0$ this pole is determined by the equation [29]

$$
p^{2}+\Pi_{\mathrm{T}}\left(p_{0}=0, p\right)=p^{2}-g^{2} N_{c} T \frac{8+(\xi+1)^{2}}{64} p
$$

where $\xi$ is the gauge parameter of covariant gauges. ${ }^{9}$ It is evidently unphysical and appears only at the non-perturbative magnetic mass scale $g^{2} T$, which contributes to the pressure starting at order $g^{6} T^{4}$. This suggests that we can in fact eliminate the entire problem by adding by hand a magnetic mass term to the transverse self-energy in Eq. (3.6)

$$
\Pi_{\mathrm{T}}(P) \rightarrow \Pi_{\mathrm{T}}(P)+m_{\mathrm{mag}}^{2}
$$

with (for $\xi=1)$

$$
m_{\mathrm{mag}}=c_{f} \frac{3}{32} g^{2} C_{A} T
$$

and $c_{f} \geq 1$, which only has an effect on the pressure beyond $O\left(g^{4}\right)$. Indeed, comparing with the effective magnetic mass for nonzero frequencies, Eq. (2.9), we find that the magnetic screening behaviour is modified only for frequencies $p_{0} \lesssim g^{4} T$ when $\mu \sim T$ and even $p_{0} \lesssim g^{4} T\left(T^{2} / \mu^{2}\right)$ when $T \ll \mu$. Note, however, that the introduction of this magnetic mass for the transverse self-energy alters the UV behavior of $p_{\text {ring }}^{\text {finite }}$, implying that both $p_{\text {ring }}^{\text {finite }}$ and $p_{\text {ring }}^{\mathrm{IR}}$ have to be modified to account for this reorganization. In $p_{\text {ring }}^{\text {finite }}$, this change is crucial because it renders the result finite, but for the already finite $p_{\text {ring }}^{\mathrm{IR}}$ the effects are beyond the order of interest (see App. D).

The numerical evaluation of $p_{\text {ring }}^{\text {finite }}$ is performed along the lines of Refs. [15, 32], with the sum over Matsubara frequencies being converted to an integration in the usual way (see e.g. Ref. [33]). Contributions containing the bosonic distribution function $n_{b}$ are best evaluated in Minkowski

\footnotetext{
${ }^{9}$ Replacing the ordinary one-loop gluon self-energy by one that includes resummation of the Debye mass does not cure the problem, but only produces a different gauge-dependent spacelike pole [30, 31].
} 
space, as UV problems are cut off by $n_{b}$, while the other contributions are evaluated in Euclidean space in order to numerically exploit the Euclidean invariance of UV contributions. By varying the parameter $c_{f}$ in Eq. (3.24), we can verify that the effects of this infrared regulator are indeed beyond the order $g^{4}$ we are aiming at. The remaining part, however, gives rise to yet another type of unphysical pole, which (at least in the long-wavelength limit) has been well-known since the earliest perturbative calculations in finite-temperature QCD [34]: in covariant gauges, the one-loop gluon self-energy, evaluated at the location of the poles corresponding to time-like propagating plasmon modes, gives rise to a (gauge-dependent) damping constant $\propto g^{2} T$ with negative sign (for all gauge parameters $\xi$, though not in Coulomb or axial gauges [35, 36]). A consistent systematic calculation of the plasmon damping constant to order $g^{2} T$ requires the use of a HTL-resummed gluon selfenergy which finally leads to a positive and gauge-independent result [31, 37]. The corresponding pole is then on the unphysical sheet where it would cause no problem for the evaluation of $p_{\text {ring }}^{\text {finite }}$. With the bare one-loop gluon self-energy appearing in our integrand we, however, have poles on the physical sheet, connected to the light-cone by a branch cut, and we need to avoid them by deforming the contour of the numerical integration in Minkowski space as sketched in Fig. 18. The details of this procedure and the entire numerical calculation are described further in Appendix D.

\section{NUMERICAL RESULTS}

Having the result of Eq. (3.17) for the QCD pressure now finally at hand, we move on to examine it numerically by evaluating the function $p_{\text {ring }}^{\text {safe }}$ using methods reviewed in Appendix $\mathbb{D}$ and adding to it the analytic part of Eq. (3.20). The sum total we call $p_{\text {IV }}$ as a reminder that its accuracy is of order $g^{4}$ for all $T$ and $\mu$, while it also includes incomplete and gauge dependent higher-order contributions. For the most part of the following analysis, we shall explicitly eliminate the latter effects by either considering sufficiently small values of $g$ or performing numerical expansions of our results in powers of $g$.

We begin by inspecting the region where the temperature is parametrically larger than the Debye scale and the results of dimensional reduction should be applicable, then continue towards making contact with HDL results on non-Fermi liquid behavior at $T \lesssim m_{\mathrm{D}}$, and finally the FreedmanMcLerran result for $T \rightarrow 0$. In all plots of the present section we use the values $N_{\mathrm{c}}=3, N_{\mathrm{f}}=2$. Because of the latter, we conveniently have $T_{F}=1$ and therefore $\tau=\bar{\tau}$ for the reduced temperature variables introduced in Eqs. (2.5) and (2.12), respectively.

\section{A. T parametrically larger than $m_{\mathbf{D}}$}

The first non-trivial check on our result - and that of dimensional reduction - is to verify that their predictions for the pressure agree to order $g^{4}$ for all temperatures and chemical potentials that are of equal parametric order in $g$. This is particularly important in order to clarify that the (entirely correct) statement in the literature about dimensional reduction being valid as long as $\pi T$ is the largest dynamical energy scale does not imply a condition $\pi T>\mu$, but rather $\pi T \gg m_{\mathrm{D}}$ (or even $\pi T \gtrsim m_{\mathrm{D}}$, as we shall find to be sufficient below). To this end, we start from the most widely studied region of $\mu=0$ by comparing our numerical result to that of the analytic one of dimensional reduction, and then increase the $\mathcal{O}\left(g^{0}\right)$ value of $\mu / T$ up to $\mu \gg \pi T$ while still having $\pi T \gg m_{\mathrm{D}} \sim g \mu$.

The results of this comparison are shown in Fig. 4, where we plot the order $g^{4} \ln g$ and $g^{4}$ contributions of the ring sum of Eq. (3.6) to the pressure together with the same quantity extracted from the result of dimensional reduction (obtained by subtracting the analytic part of our result from the DR one). The agreement is perfect up to the numerical accuracy of our result, and only 


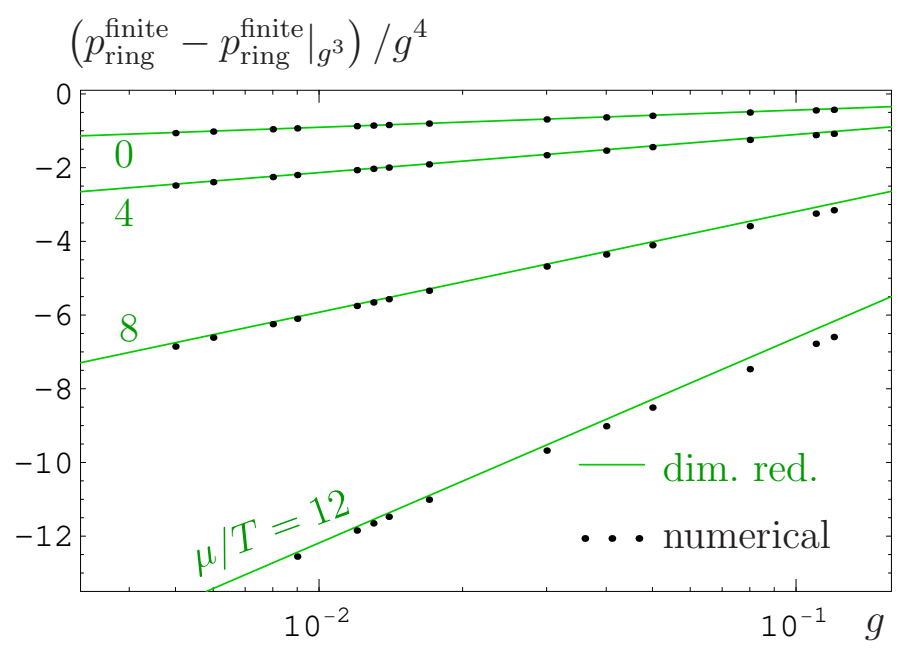

FIG. 4: Comparison of the $g^{4} \log g$ and $g^{4}$ terms of the numerical computation and the analytic DR result, for various values of $\mu / T$. The perturbative terms are subtracted up to order $g^{3}$.

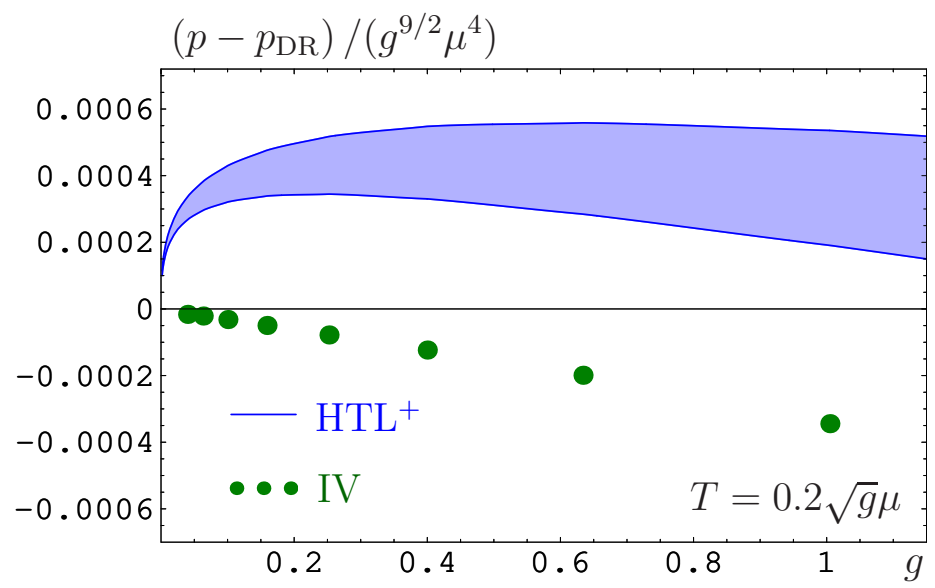

FIG. 5: Comparison of the HTL+ pressure and our numerical result $p_{\text {IV }}$ in the region of $T=\tau \sqrt{g} \mu, \tau=0.2$, with the known perturbative terms from dimensional reduction subtracted and the entire quantities divided by $g^{9 / 2}$. This plot shows that both the HTL+ result and our numerical one are accurate at least up to order $g^{9 / 2}$. The renormalization scale has been varied between $\mu$ and $4 \mu$. While $p_{\mathrm{IV}}-p_{\mathrm{DR}}$ is scale independent, $p_{\text {HTL }+}-p_{\text {DR }}$ has a scale dependence at order $g^{4} \mu^{2} T^{2} \sim g^{5} \mu^{4}$.

at larger values of $g$ can one see that the agreement is getting slightly worse with increasing $\mu / T$. This was, however, to be expected, since there $\mu / T$ is coming closer to the value $g^{-1}$, making $m_{\mathrm{D}} / T$ of order one which is parametrically the limit of applicability of dimensional reduction. Our conclusion is that the result of dimensional reduction is valid at in principle arbitrarily large $\mathcal{O}\left(g^{0}\right)$ values of $\mu / T$, though the expansion in $g$ only makes sense at smaller and smaller values of $g$ as this parameter is increased. This statement will be made more concrete in the following sections.

The logical next step is to test the validity of dimensional reduction at temperatures larger than but now parametrically closer to the Debye scale. For concreteness, we specialize to the case of $T \sim \sqrt{g} \mu$, for which the prediction of dimensional reduction is given in Eq. (2.6). In this region, 


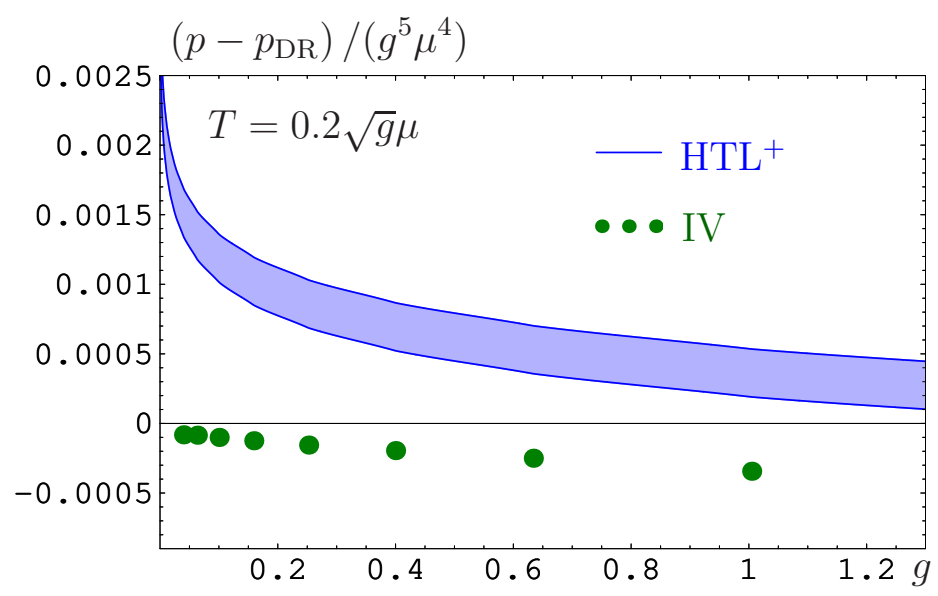

FIG. 6: Same as Fig. 5 but normalized to $g^{5}$. While the HTL+ result is no longer accurate to this order and diverges logarithmically, our numerical result still correctly reproduces the dimensional reduction result for the pressure at order $g^{5} \mu^{4}$.

the error in our result is of order $g^{11 / 2} \mu^{4}$ and that of the minimal HTL resummation $g^{5} \mu^{4}$, so that the first one should be able to reproduce the first seven and the latter the first six terms of the series (2.6). And indeed, a numerical evaluation of both Eqs. (3.17) and (2.14) and the subtraction of the first terms of Eq. (2.6) shows the expected results: as displayed in Fig. (5) we find perfect agreement in comparing the dimensional reduction result with the HTL one (2.15) and with that of our new approach up to order $g^{9 / 2}$. In Fig. 6 we see that our numerical evaluation of $p_{\text {IV }}$ is accurate enough to even verify the $g^{5} \mu^{4}$ term in the dimensional reduction result, while the HTL result starts deviating from the DR one at this order.

\section{B. $T$ comparable to $m_{\mathbf{D}}$}

In Figs. 7 [ 9] we plot the the temperature-dependent contributions to the interaction pressure $\delta p$ (see Eq. (2.11)) for $T \sim m_{\mathrm{D}}$ as extracted from our numerical calculation of $p_{\mathrm{IV}}$ but with no expansions in powers of $g$. We compare this with $p_{\mathrm{HTL}+}$ as well as with the dimensional reduction result expanded to orders $g^{2}, g^{3}, g^{4}$ and $g^{5}$ which refer to the counting in powers of $g$ when $T \sim \mu$. For $T \sim g \mu$, however, the terms $g^{2} \mu^{2} T^{2}, g^{3} \mu^{3} T$, and $g^{4} \mu^{4} \ln T$ all become of the same order of magnitude and together constitute the leading temperature-dependent contribution to the interaction pressure $p-p_{\mathrm{SB}}$ which is contained in the result marked by the dashed line " $g^{4}$ ". For completeness, we also include the complete dimensional reduction result to (explicit) order $g^{5}$, but it should be remembered that the term $g^{5} T \mu^{3}$ is already of the same magnitude as the unknown $g^{6} \mu^{4}$ piece when $T \sim g \mu$, and is therefore both incomplete and beyond our scope which also explains why the $g^{4}$ curve seems to produce better agreement with our results than the $g^{5}$ one.

The different results are normalized to the leading term of the $T$-dependent part of the interaction pressure in the dimensional reduction result (A1),

$$
\delta p_{\mathrm{DR}}^{(2)}=-g^{2} d_{A}\left\{\frac{T_{F}}{16 \pi^{2}} \mu^{2} T^{2}+\frac{5 T_{F}+2 C_{A}}{244} T^{4}\right\} .
$$

To understand the structure of these figures, note that the $g^{3}$ curve goes like $-1+(4 / 3 \pi) m_{\mathrm{D}} / T$ for small $T$ and like $-1+1.07 \mathrm{~g}$ for large $T$. At $T \ll m_{\mathrm{D}}$ it, of course, deviates from the exact 


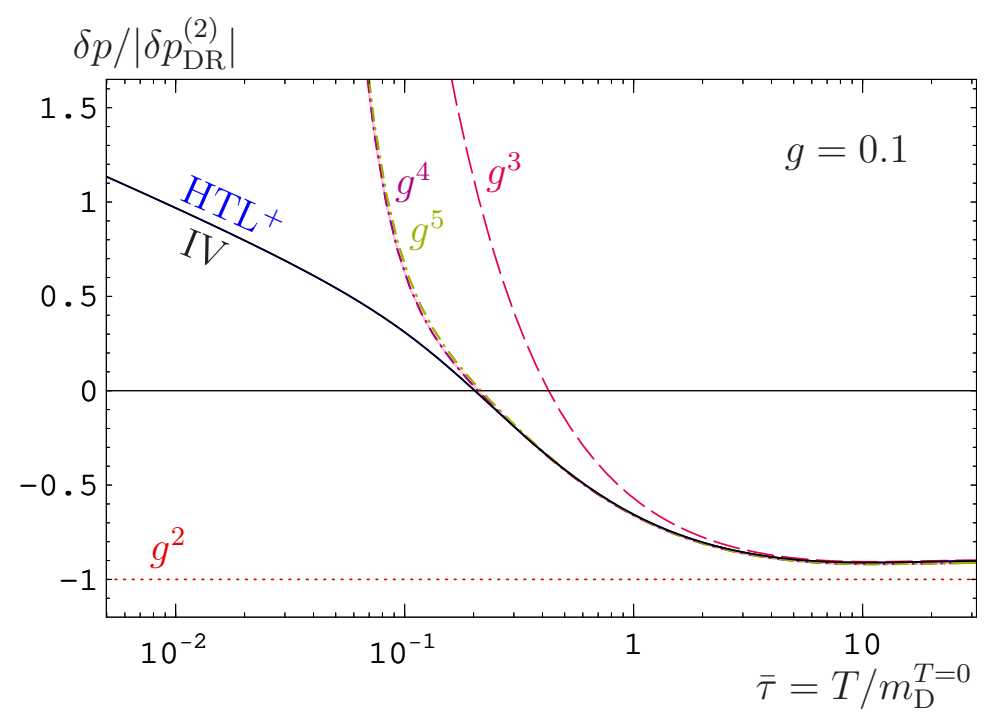

FIG. 7: Thermal contribution to the interaction pressure $\delta p$ as a function of $T / m_{\mathrm{D}}^{T=0}$ for fixed chemical potential $\mu$ and coupling $g=0.1$. For this value of the coupling, the results of the numerical evaluation of $p_{\text {anl }}+p_{\text {ring }}^{\text {safe }}$ and $\mathrm{HTL}^{+}$coincide within plot resolution. The result is compared to the dimensional reduction pressure at orders $g^{2}, g^{3}, g^{4}$, and $g^{5}$ (where the latter is included only for completeness, as neither $p_{\mathrm{IV}}$ nor $p_{\mathrm{HTL}^{+}}$contain contributions of order $g^{5}$ ). The effect of varying the renormalization scale $\Lambda_{\overline{\mathrm{MS}}}=\mu \ldots 4 \mu$ is not visible for this value of the coupling.

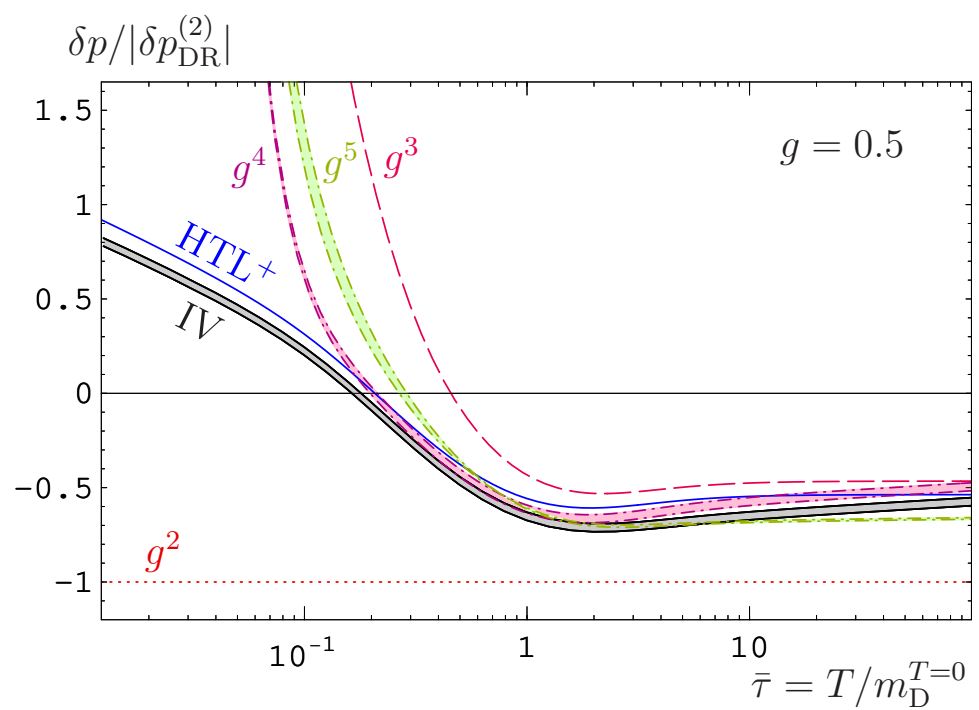

FIG. 8: Same as Fig. [7 but for $g=0.5$. The results of the numerical evaluation of $p_{\text {anl }}+p_{\text {ring }}^{\text {safe }}$ and нтL ${ }^{+}$ can now be distinguished due to their different content of higher-order terms. When two lines of the same type run close to each other, they differ by changing the renormalization scale $\Lambda_{\overline{\mathrm{MS}}}=\mu \ldots 4 \mu$.

result which is instead dominated by the leading $\frac{2}{9} \ln T^{-1}$ behavior of the low-temperature series of Eq. (2.13) when normalized by the absolute value of Eq. (4.1).

For small values of $g \sim 0.1$, Fig. 7 shows that the numerical evaluation of Eq. (3.17) perfectly agrees with the result of the HTL resummation (the two curves lie virtually on top of each other). At this value of $g$, also the complete dimensional reduction result to (explicit) order $g^{5}$ is virtually 


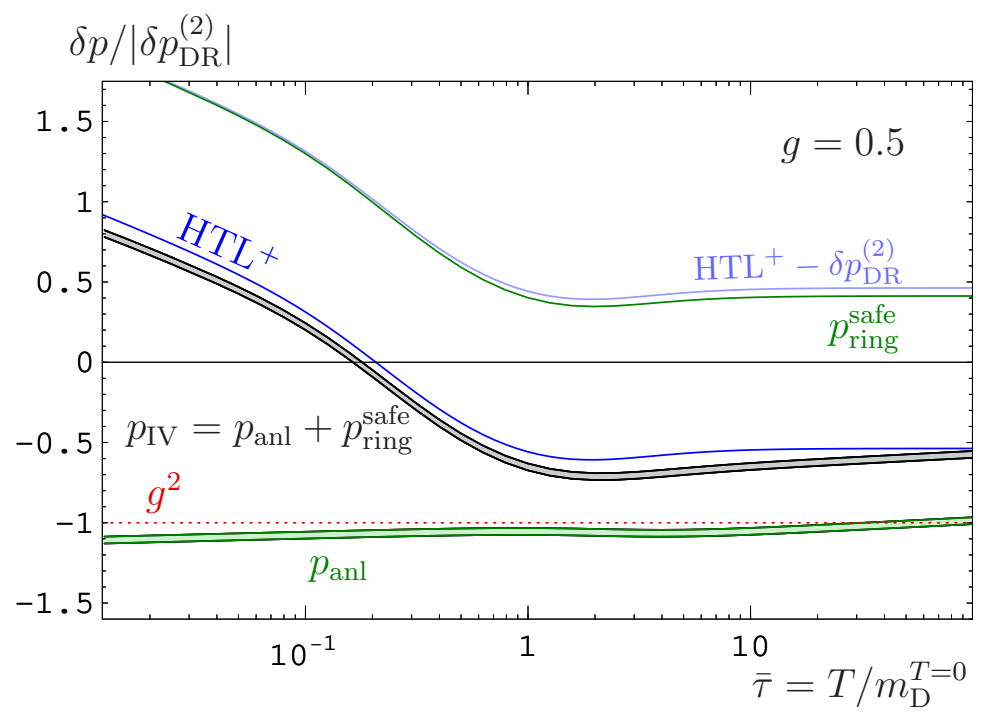

FIG. 9: Same as Fig. 8 but with $p_{\text {IV }}$ separated into $p_{\text {anl }}$ and $p_{\text {ring }}^{\text {safe }}$. As the $g^{4}$ contribution in $\delta p_{\text {anl }}$ only amounts to a small correction (of effective order $g^{6}$ ), the shape of the full pressure curve as a function of $T$ (beyond the rather trivial $g^{2}$ contribution) is mainly determined by $p_{\text {ring }}^{\text {safe }}$. The renormalization scale dependence $\Lambda_{\overline{\mathrm{MS}}}=\mu \ldots 4 \mu$ is entirely due to $p_{\text {anl }}$.

indistinguishable from the order $g^{4}$ result. The dimensional reduction result reproduces the numerical results remarkably well down to temperatures of about $0.2 m_{\mathrm{D}}^{T=0}$, but at even lower $T$ severely overestimates the logarithmic growth of $\delta p / T^{2}$ as $T \rightarrow 0$. This is to be expected, since, in the limit $T \rightarrow 0$, the plasmon term of order $g^{3} \mu^{3} T$ in the pressure is clearly unphysical, as it would lead to a nonvanishing entropy at $T=0$; the $g^{4} \mu^{4} \ln T$ term of the dimensional reduction result, while evidently crucial for good agreement down to $T \approx 0.2 m_{\mathrm{D}}$, would even lead to a diverging entropy as $T \rightarrow 0$. The point at which the dimensional reduction result ceases to be a good approximation for both $p_{\mathrm{HTL}+}$ and $p_{\mathrm{IV}}$ seems to agree rather well with the value of $T / m_{\mathrm{D}}$ where $\delta p$ switches sign.

In Fig. 8 we consider a larger coupling $g=0.5$, for which we begin to see effects from varying the renormalization scale $\Lambda_{\overline{\mathrm{MS}}}$ in our result by a factor of 2 around the central value $2 \mu$, except in the HTL+ result, where $\Lambda_{\overline{\mathrm{MS}}}$ appears only in the $T=0$ (Freedman-McLerran) part of the result. ${ }^{10}$ For small $T / m_{\mathrm{D}}^{T=0}$, we find good agreement between the HTL+ result and $p_{\text {IV }}$, with the dimensional reduction result to order $g^{4}$ lying in between the two in the range $T / m_{\mathrm{D}}^{T=0} \approx 0.1 \ldots 10$, but deviating again abruptly for $T / m_{\mathrm{D}}^{T=0}<0.2$, which is where $\delta p$ changes sign. At this value of the coupling, the complete order $g^{5}$ result of dimensional reduction is still reasonably close to the order $g^{4}$ result. While it is certainly unreliable when $\delta p>0$, the order $g^{5}$ result suggests that taking into account the next higher orders in $g$ may move the onset of non-Fermi-liquid behavior to slightly larger $T / m_{\mathrm{D}}$.

Fig. 9 shows how the final result $\delta p_{\text {IV }}$ is composed of the infrared-safe piece $p_{\text {anl }}$ and the ring sum $p_{\text {ring }}^{\text {safe }}$. At parametrically small $T \sim g \mu$, the $T$-dependent terms in the interaction part of $p_{\text {anl }}$ which are of effective order $g^{4} \mu^{4}$ come just from the terms $g^{2} T^{2} \mu^{2}$, so that the shape of $\delta p$ in the

${ }^{10}$ In Fig. 8 the value $g=0.5$ is kept fixed for all $\Lambda_{\overline{\mathrm{MS}}}$, which means that the $x$-axis does not correspond to a renormalization-group invariant variable. The (explicit) dependence of the results on $\Lambda_{\overline{\mathrm{MS}}}$ is here shown only to assess the theoretical error in the numerical comparison between the different approaches. Taking into account the implicit $\Lambda_{\overline{\mathrm{MS}}}$ dependence of $g$, the scale dependence of all the results we are comparing is of the order of their error, which is $O\left(g^{6} \mu^{4}\right)$ at $T \sim g \mu$. 


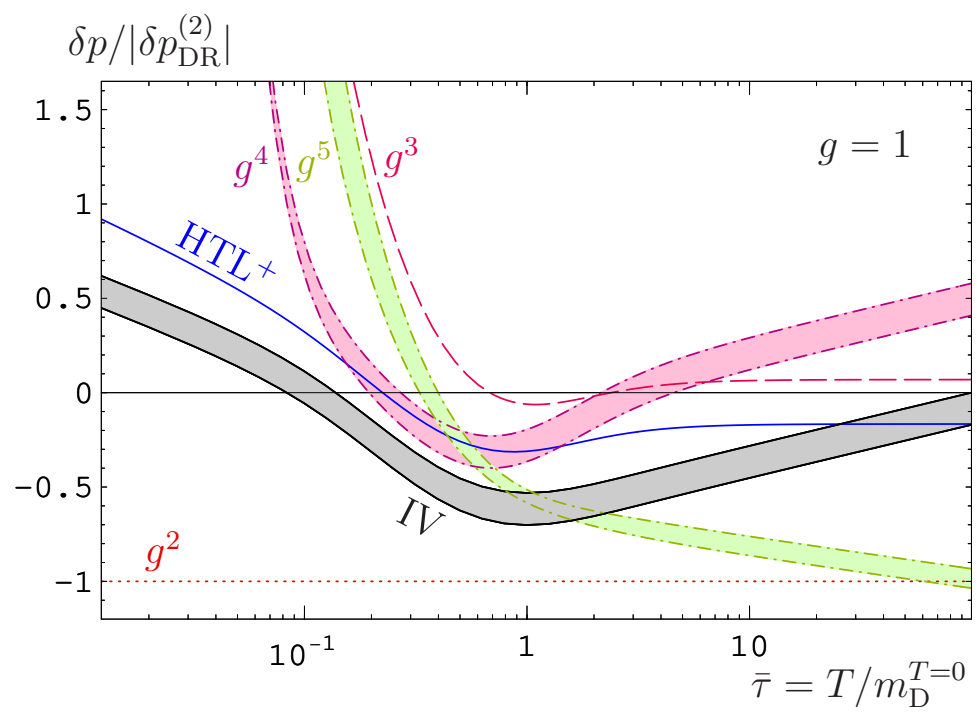

FIG. 10: Same as Fig. 7 but for $g=1$. At this value of the coupling, the numerical result for $p_{\text {IV }}$ begins to be visibly affected by the choice of the magnetic mass (3.24) which here is taken with $c_{f}=1$.

above figures is mainly determined by $\delta p_{\text {ring }}^{\text {safe }}$ which is seen to coincide with $\delta p_{\mathrm{HTL}+}-\delta p_{\mathrm{DR}}^{(2)}$ up to terms beyond $g^{4}$ accuracy.

Finally, in Fig. 10 we consider $g=1$ which is roughly the value of the QCD coupling at $100 \mathrm{GeV}$. Here the result for $p_{\mathrm{IV}}$ still follows $p_{\mathrm{HTL}+}$ for $T<m_{\mathrm{D}}$ and the $g^{4}$ result of dimensional reduction for $T \gg m_{\mathrm{D}}$, but there is an overall shift due to the (incomplete and gauge dependent) order $g^{6} \mu^{4}$ terms in $p_{\mathrm{IV}}$. At this value of $g$ one also notices that the dimensional reduction result to order $g^{4}$ deviates rather strongly from the result to order $g^{5}$, thus showing poor apparent convergence, in particular at high $\tau$.

For the remainder of the discussion of our numerical results at $T \sim m_{\mathrm{D}}$, we concentrate on the contributions of order $g^{4} \mu^{4}$ to the pressure and explicitly discard all terms beyond this accuracy, as this helps us to better analyze the breakdown of dimensional reduction observed in the three previous plots. To this end, we consider the difference of the $g^{4} \mu^{4}$ term in the DR result at $T \sim g \mu$ and the corresponding piece in the Freedman-McLerran zero-temperature expression. A straightforward evaluation of this quantity gives

$$
\begin{aligned}
\frac{1}{d_{A} \mu^{4}} \delta p^{\mathrm{DR}}\left(\mu, \bar{\tau}=\frac{T}{m_{\mathrm{D}}}\right) & =-\frac{\bar{g}^{4} \bar{\tau}^{2}}{16 \pi^{4}}+\frac{\bar{g}^{4} \bar{\tau}}{12 \pi^{5}} \\
& -\frac{\bar{g}^{4}}{768 \pi^{6}}\left[33-3 \delta-12 \gamma-2 \pi^{2}+2 \ln 2(8 \ln 2+7)+12 \ln (\pi \bar{\tau})\right]+O\left(g^{6}\right)
\end{aligned}
$$

which, like the $\mathrm{HDL}^{+}$result, only depends on $g$ and $T$ to order $g^{4}$ through the combinations $\bar{g} \equiv T_{F}^{1 / 2} g$ and $\bar{\tau}=\pi T /(\bar{g} \mu)=T / m_{\mathrm{D}}$ and where $\delta$ is the numerical constant appearing in Eq. (2.7). As we have seen, in this region the $g^{4}$ content of our $p_{\mathrm{IV}}$ agrees perfectly with that of $p_{\mathrm{HDL}^{+}}$defined by Eqs. (2.10) and (2.11). Therefore, to simplify our numerical efforts, we compare in Fig. 11] the above function with the $\mathrm{HDL}^{+}$result for the same quantity $\delta p$, after by hand subtracting the terms proportional to $\bar{\tau}^{2}$ and $\bar{\tau}$ of Eq. (4.2) from both results (which correspond to the terms of order $g^{2} \mu^{2} T^{2}$ and $g^{3} \mu^{3} T$ in the expansion of the pressure). ${ }^{11}$ This helps us to expose the term of

${ }^{11}$ Note that these terms are not present in the $\mathrm{HDL}^{+}$result at small values of $\bar{\tau}$ (but that this does not matter, as 


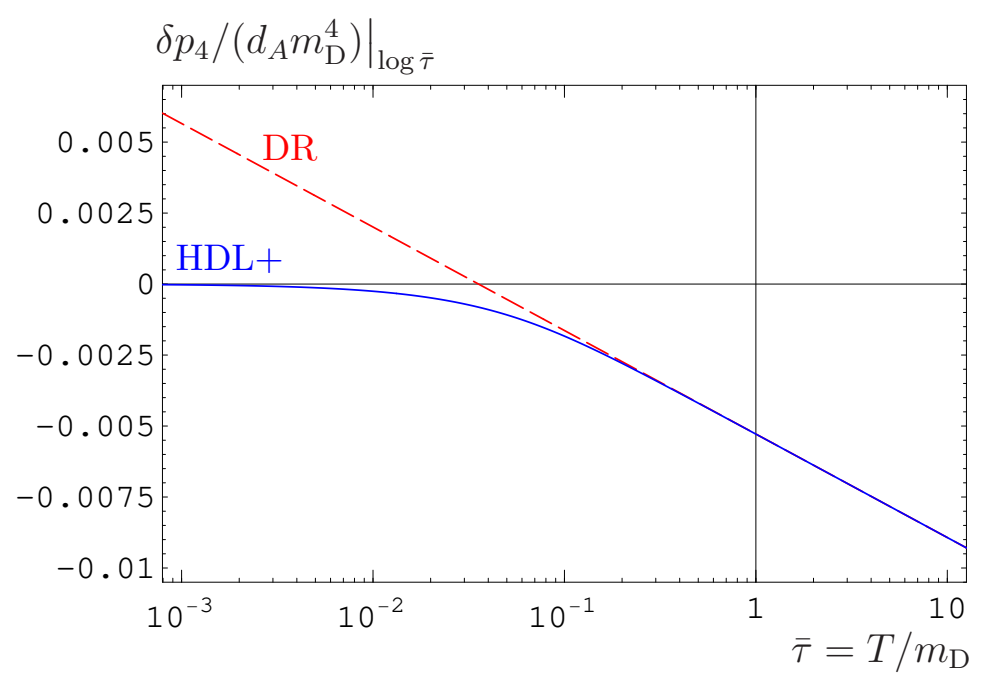

FIG. 11: Plot of the last term in Eq. (4.2) (dashed line) in comparison with $\delta p_{\mathrm{HDL}^{+}}$with the first two terms of Eq. (4.2) subtracted, in units of $m_{\mathrm{D}}^{4}=\bar{g}^{4} \mu^{4} / \pi^{4}$ and as a function of $\log _{10}(\bar{\tau})$.

order $g^{4} \mu^{4} \ln T$, whose divergence at $T \rightarrow 0$ signals the failure of dimensional reduction to correctly describe the zero-temperature limit of the pressure.

From Fig. 11] we observe that for $\bar{\tau} \gtrsim 0.2 m_{\mathrm{D}}$ there is a $\ln \bar{\tau}$ term in the $\mathrm{HDL}^{+}$result which agrees perfectly with that of Eq. (4.2). This fact turns out to have a natural explanation which gives us important insight into the breakdown of dimensional reduction. The key observation is that the ultimate reason for this breakdown lies in the incorrect treatment of low-temperature IR divergences in DR: in deriving its prediction for the QCD pressure, one assumes that the temperature acts as the sole IR cut-off for all non-zero bosonic Matsubara frequencies in the logarithmically IR divergent three-loop ring diagrams, so that for them no resummations are necessary. ${ }^{12}$ While this indeed is justified for $T \gg m_{\mathrm{D}}$, there is an obvious problem in the region $T \sim m_{\mathrm{D}}$, ultimately leading to the diverging of the DR result in the zero-temperature limit where discrete Matsubara modes no longer exist. In a physically consistent calculation, where one performs a resummation also for the non-static modes, the logarithm of temperature in the dimensional reduction result above gets replaced by one of a true IR regulator $T \times R(g \mu / T)$, where the function $R$ is linear at large values of its argument and approaches a non-zero constant as $g \mu / T \rightarrow 0$. Unlike $T$, this regulator therefore does not vanish at $T=0$ which explains the finite $\bar{\tau} \rightarrow 0$ limit of the $\mathrm{HDL}^{+}$curve in Fig. 11. However, at large values of $\bar{\tau}$ the logarithm of $T R$ gives rise to the $\ln \bar{\tau}$ behavior with the same coefficient as in the dimensional reduction result which is what we observe in Fig. 11]

\section{C. $T$ smaller than $m_{\mathrm{D}}$}

In the limit $\bar{\tau}=T / m_{\mathrm{D}} \rightarrow 0$, our evaluation of $p_{\mathrm{IV}}$ approaches the Freedman-McLerran result (2.7) as shown in Figs. 12 and 13 , where the difference of $p_{\text {IV }}(T=0)$ and $p_{\text {FMcL }}$ is plotted as a function of $g$. The agreement to order $g^{4}$ and the absence of a $g^{5}$ term is shown in Fig. 12, where we normalize the result by $g^{5} \mu^{4}$. Fig. 13 on the other hand shows that $p_{\text {IV }}$ differs from the

\footnotetext{
their effect in Fig. 11 in any case vanishes as $\bar{\tau} \rightarrow 0$ ).

12 Which amounts to expanding the already resummed propagators for the non-static gluon modes in these diagrams in powers of $g \mu / T$.
} 


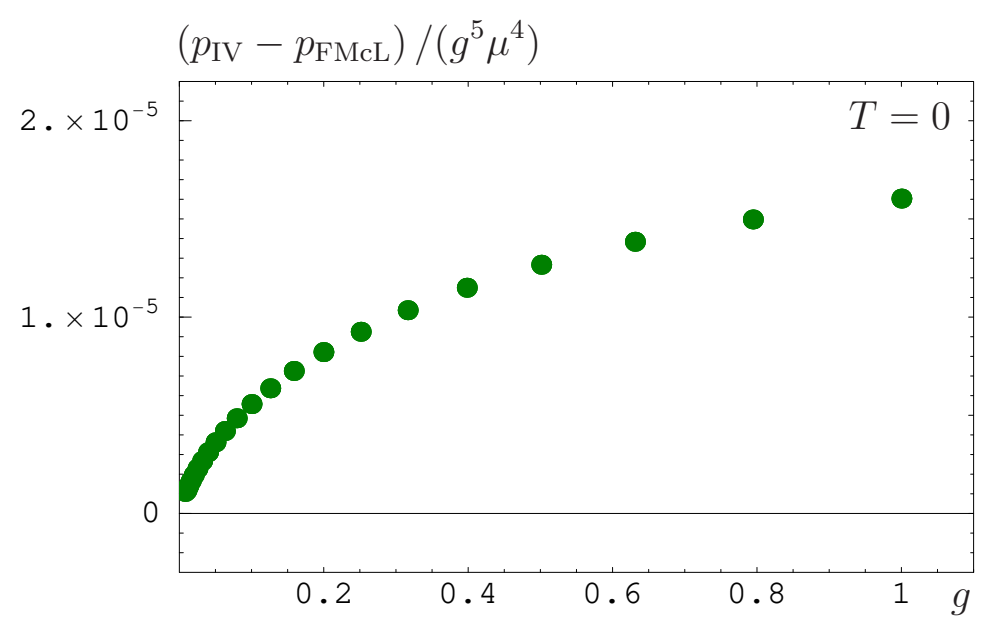

FIG. 12: Comparison of our numerical result $p_{\text {IV }}$ and the Freedman-McLerran result. The plot shows agreement to order $g^{5}$, i.e., agreement in the nonvanishing coefficients up to and including order $g^{4}$, and the absence of order $g^{5}$ contributions.

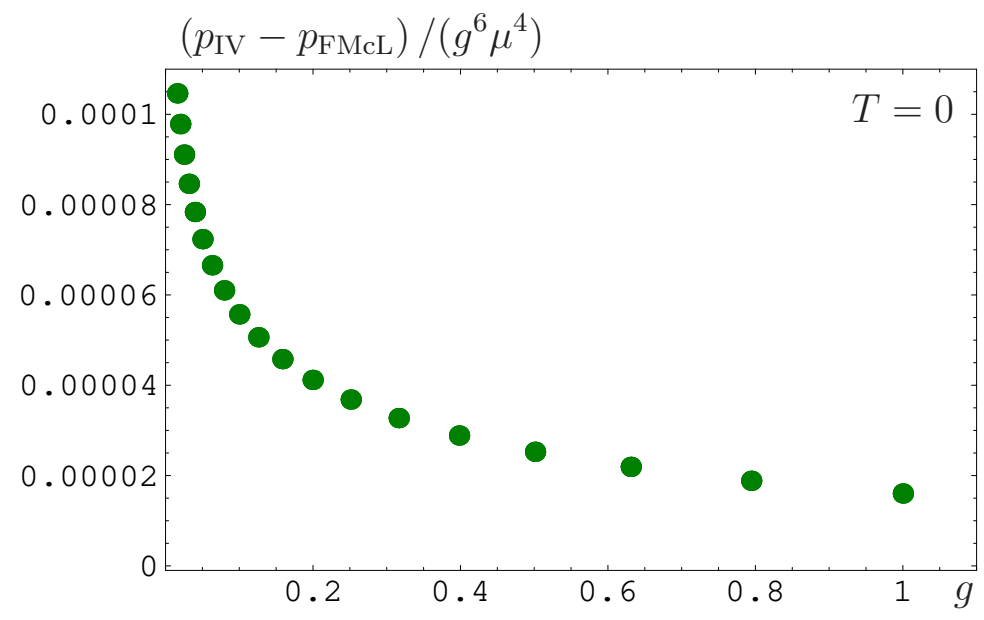

FIG. 13: Same as Fig. 13 but divided by $g^{6}$ instead of $g^{5}$, revealing that $p_{\text {IV }}$ contains a term of order $g^{6} \ln g \mu^{4}$, which is however incomplete as it is beyond the accuracy of our setup.

Freedman-McLerran result at order $g^{6}$, where it contains a term of order $g^{6} \ln g$. This, however, is incomplete, as the true (unknown) $g^{6} \ln g$ term in the $T=0$ pressure gets contributions also from the two-loop gluon self energy.

In Fig. 14 we plot the coefficients of the low-temperature pressure in the perturbative expansion

$$
\frac{p}{\mu^{4}}=p_{0}+\frac{g^{2}}{4 \pi} p_{2}+\frac{g^{4}}{(4 \pi)^{2}}\left(p_{4}+p_{4}^{\prime} \log g\right)+\mathcal{O}\left(g^{6} \ln g\right)
$$

as a function of $\tau=\pi T /(g \mu)$ for $N_{c}=3$ and $N_{f}=2$. Here, we have again numerically evaluated the $\mathrm{HDL}^{+}$result which as we have shown agrees with our new approach to order $g^{4}$ for $T \sim g \mu$. For $\tau=0$ the coefficients reproduce the Freedman-McLerran result, whereas for larger $\tau$ the coefficients are dominated by Stefan-Boltzmann contributions $T^{2} \mu^{2} \sim g^{2} \mu^{4}$ and $T^{4} \sim g^{4} \mu^{4}$. 


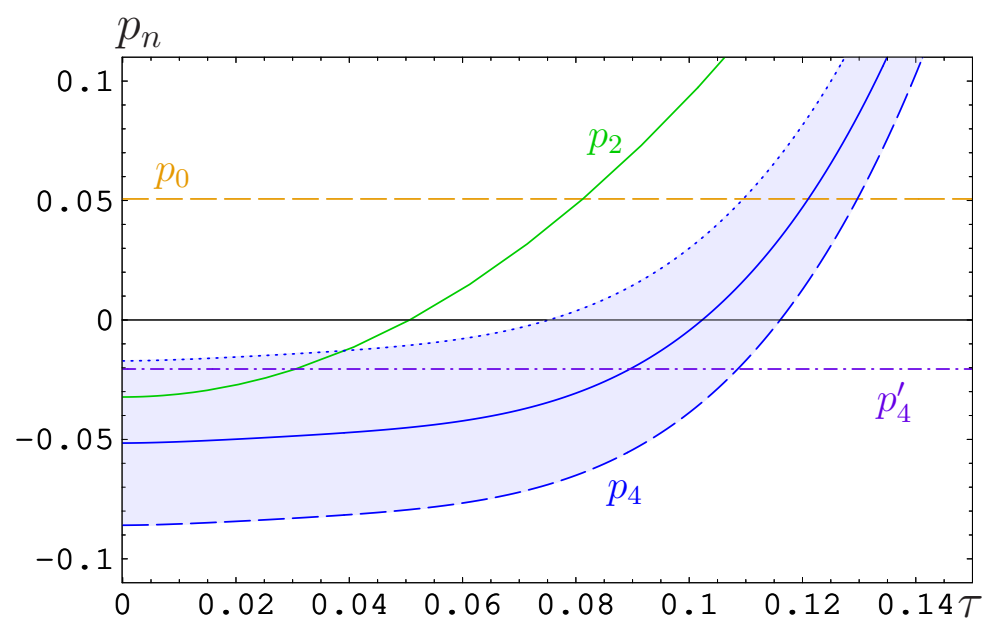

FIG. 14: Dimensionless coefficients of the expansion of the pressure in powers of $g$ at $\pi T=\tau g \mu$ as a function of $\tau$ for $N_{c}=3, N_{f}=2$. The coefficients $p_{0}$ and $p_{4}^{\prime}$ are constants, $p_{2}$ is quadratic in $\tau$, while $p_{4}$ shows a $\tau^{2} \log \tau$ behavior for small $\tau$. The renormalization scale is varied through $\Lambda_{\overline{\mathrm{MS}}}=\mu \ldots 4 \mu$.

In Fig. 15] we compare the $T$-dependent contribution to the interaction pressure, $\delta p$, with the extrapolation of the dimensional reduction result (where $p_{\mathrm{FMcL}}$ has been subtracted since $p_{\mathrm{DR}}$ does not exist at $T=0)$ to $T \ll m_{\mathrm{D}}$ in a linear plot. Here, $\delta p$ is normalized by $m_{\mathrm{D}}^{4} \sim g^{4} \mu^{4}$ rather than divided by a term quadratic in temperature to better show its absolute magnitude ${ }^{13}$. As could already be seen in the previous plots, the dimensional reduction result works surprisingly well down to temperatures where $\delta p$ changes sign which happens at $T \approx 0.2 m_{\mathrm{D}}$. At even lower temperatures, the dimensional reduction result overestimates the leading positive contribution to $\delta p$ which in reality goes to zero like (or grows $\sim \log T$ with the normalization of Figs. 7] 9)

$$
\delta p(\mu, T \ll g \mu)=\frac{d_{A} T_{F}}{72 \pi^{2}} g^{2} \mu^{2} T^{2} \ln \frac{c T_{F}^{1 / 2} g \mu}{T}+O\left((g \mu)^{4 / 3} T^{8 / 3}\right)
$$

with the constant $c \approx 0.284794$. This expression determines the total pressure of the normal QCD phase as

$$
p_{\text {normal }}(\mu, T \ll g \mu)=p_{\text {normal }}(\mu, 0)+\delta p(\mu, T \ll g \mu),
$$

where $p_{\text {normal }}(\mu, 0)$ is given in Eq. (2.7).

For exponentially small $T$ with $\ln (g \mu / T) \gtrsim g^{-2}$ the contribution of Eq. (4.4) can even be larger than the leading $T$-dependent ${ }^{14}$ term $\propto \mu^{2} T^{2}$ in the Stefan-Boltzmann pressure [38]. However, before one reaches temperatures so small that $\ln (g \mu / T) \gtrsim g^{-2}$ in a nonabelian plasma, one encounters the nonperturbative pair instability of color superconductivity and the formation of a gap 13. $\phi \sim T_{c} \sim \mu g^{-5} \exp \left[-3 \pi^{2} /(g \sqrt{2})\right]$ at the parametrically larger temperatures with $\ln \left(g \mu / T_{c}\right) \gtrsim g^{-1}$. In our (resummed) perturbative approach, we will not directly encounter this nonperturbative instability, but since at the superconducting transition temperature the pressures of the normal and superconducting phases in any case have to be equal, it is of some interest to evaluate the

13 The normalization to $\delta p_{\mathrm{DR}}^{(2)}$ is however more appropriate if one is interested in the magnitude of the effects to entropy and specific heat.

14 Note that in the entropy or in the specific heat these terms constitute the leading ones. 


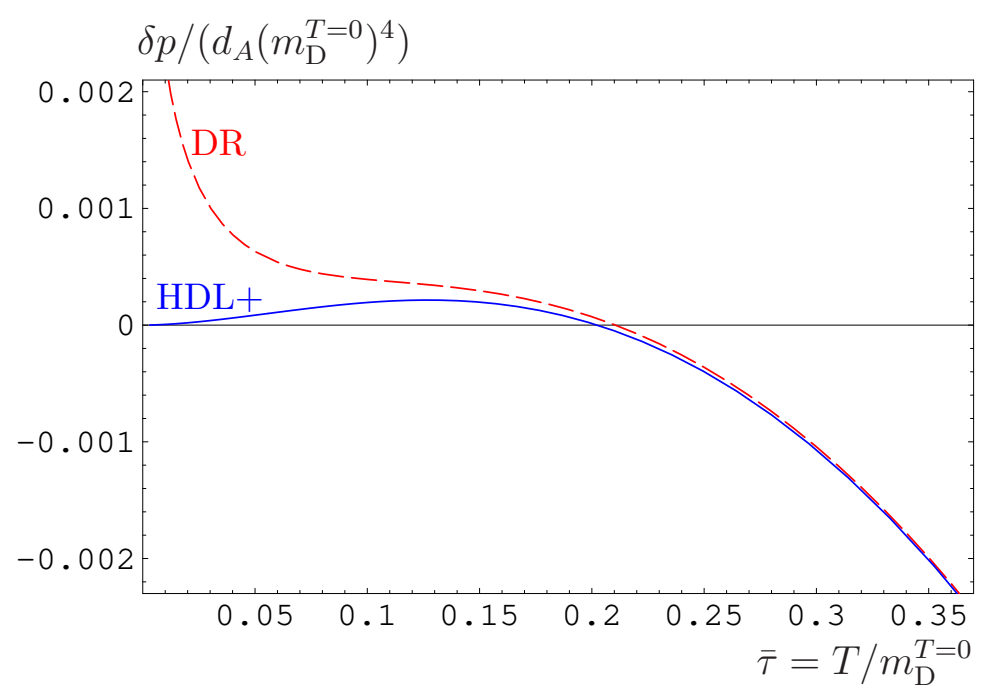

FIG. 15: Plot of the $T$-dependent part of the interaction pressure $\delta p$ (see Eq. (2.11)) to order $g^{4}$ in the regime $\pi T \lesssim m_{\mathrm{D}}$ in units of $\left(m_{\mathrm{D}}^{T=0}\right)^{4}=\bar{g}^{4} \mu^{4} / \pi^{4}$ and as a function of $\bar{\tau}=T / m_{\mathrm{D}}$. The dashed line denotes the dimensional reduction result to order $g^{4}$, and the full line the $\mathrm{HDL}^{+}$result which in this regime coincides with the order- $g^{4}$ content of $p_{\mathrm{IV}}$.

correction of Eq. (4.4) at the transition temperature $T_{c}^{\mathrm{SC}}$. Inserting $\ln (T / \mu) \approx-3 \pi^{2} /(g \sqrt{2})$ into Eq. (4.4), one finds

$$
p_{\text {normal }}\left(\mu, T_{c}^{\mathrm{SC}}\right)=p_{\text {normal }}(\mu, 0)+\frac{d_{A} T_{F}}{24 \sqrt{2}} g \mu^{2}\left(T_{c}^{\mathrm{SC}}\right)^{2} .
$$

Comparing this to the $T=0$ pressure of, e.g., the 2SC phase from [39] and expressing it in terms of $T_{c}$ by replacing $\phi \rightarrow \pi e^{-\gamma} T_{c}^{\mathrm{SC}}$, we have

$$
p_{\mathrm{SC}}(\mu, 0) \approx p_{\text {normal }}(\mu, 0)+e^{-2 \gamma} T_{F} \mu^{2}\left(T_{c}^{\mathrm{SC}}\right)^{2} .
$$

Thus we observe that at the temperature where color superconductivity sets in, the contribution of resummed perturbation theory is $g d_{A} e^{2 \gamma} /(24 \sqrt{2}) \approx 0.75 g$ times the $T=0$ contribution of the gap.

\section{SUMMARY OF PERTURBATION THEORY ON THE $\mu$ - $T$ PLANE}

As we have seen in the previous Section, the weak coupling expansion of the QCD pressure goes through changes in its form when $T / \mu$ becomes comparable to some positive power of the coupling constant $g$ and this power is then increased. In particular, at $T \lesssim g \mu$ dimensional reduction ceases to be applicable and a resummation of the nonstatic parts of the gluon self energy becomes necessary, although numerically the dimensional reduction result works surprisingly well down to the rather small value $T / m_{\mathrm{D}} \approx 0.2$. At this temperature, which to leading order in the coupling reads

$$
\frac{T_{\mathrm{NFL}}}{\mu} \approx 0.064 \sqrt{\frac{N_{f}}{2}} g
$$

the $T$-dependent contributions to the interaction pressure change sign, marking the onset of socalled non-Fermi-liquid behavior. At parametrically even smaller temperatures, one eventually 


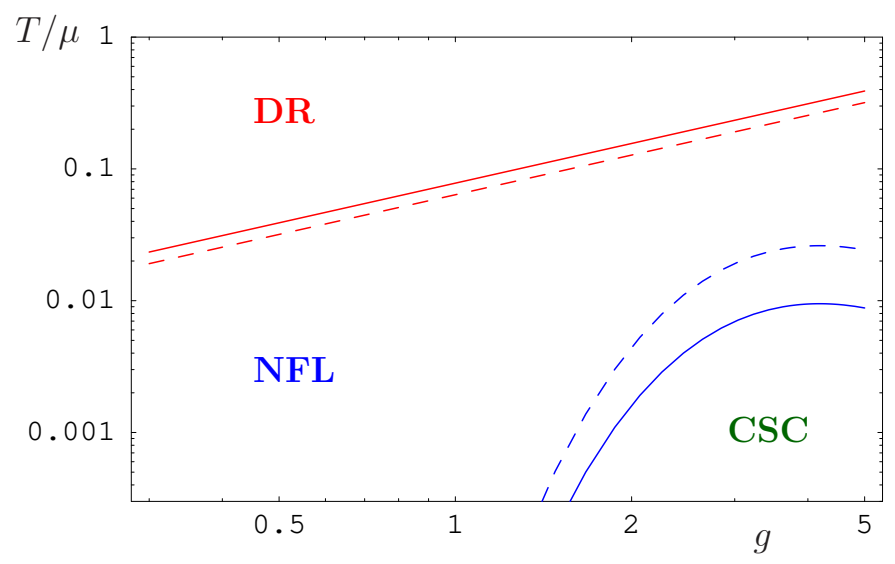

FIG. 16: The dividing line between the regime of dimensional reduction (DR) and that of non-Fermi-liquid behavior (NFL) as given by Eq. (5.11) for $N_{f}=3$ (full lines) and $N_{f}=2$ (dashed lines), in comparison with the weak-coupling result (5.2) for the critical temperature of color superconductivity (CSC) when extrapolated to large coupling.

encounters the critical temperature of color superconductivity, which has been calculated to leading order in a weak coupling analysis as [40]

$$
\frac{T_{c}^{\mathrm{SC}}}{\mu} \simeq 2 \frac{e^{\gamma}}{\pi} e^{-\left(\pi^{2}+4\right) / 8}(4 \pi)^{4}\left(\frac{2}{N_{f}}\right)^{5 / 2} g^{-5} e^{-3 \pi^{2} / \sqrt{2} g}
$$

for a spin-zero condensate (which gives the largest value of $T_{c}$ ).

In Fig. 16] we compare $T_{\mathrm{NFL}}$ and $T_{c}^{\mathrm{SC}}$ when extrapolated to large coupling (where of course correspondingly large modifications can be expected). At least for smaller coupling, i.e., sufficiently high densities, there is a clear separation of regimes in the $\mu-T$ plane with qualitatively different weak coupling descriptions.

In Fig. 17 we display the structure of the weak coupling expansion by showing how the magnitudes of its terms depend on the power $x$ in the order-of-magnitude equality $T \sim g^{x} \mu$ (which of course removes the superconducting phase from the picture). The $x$-axis in this figure corresponds to the ratio $\ln (T / \mu) / \ln (g)$ in the limit $g \rightarrow 0$ and $T / \mu \rightarrow 0$. For each value of $x$ we give the orders of the first several terms in the expansion of the pressure in powers of $g$, not counting separately terms with an extra factor of $\ln (g)$. Full lines denote known contributions, while dashed and dash-dotted lines correspond to the as-yet unknown ones.

At $x=0$, i.e. $T \sim \mu$ the situation is still the same as with $\mu=0$ : the weak coupling expansion of the pressure is organized in single powers (and logs) of $g$. The relevant effective theory is given by dimensionally reduced electrostatic QCD (EQCD) which for the pressure has been worked out up to but not including order $g^{6}$ which is where nonperturbative physics from magnetostatic QCD (MQCD) starts to contribute. This barrier is indicated by a thick red line in Fig. 17.

Because $g$ is treated as an arbitrarily small parameter, everything in Fig. 17w with the exception of the border at $x=0$ corresponds to the regime $T \ll \mu$, namely $T \sim g^{x} \mu$ with $x>0$. As long as $x<1, T$ is parametrically larger than the Debye mass $\sim g \mu$, and so dimensional reduction should still be applicable. However, each coefficient of the original series at $x=0$ now has to be expanded in powers of $T / \mu \sim g^{x}$. The 2-loop pressure contribution for example yields three different terms for $x>0$ : one is proportional to $\mu^{4}$ and thus is always of order $g^{2}$, another - proportional to $\mu^{2} T^{2}$ - gives the line $y=2+2 x$ and the third term proportional to $T^{4}$ produces the line $y=2+4 x$. Starting with the plasmon term which is of order $g^{3}$ at $x=0$, we obtain an infinite series of 


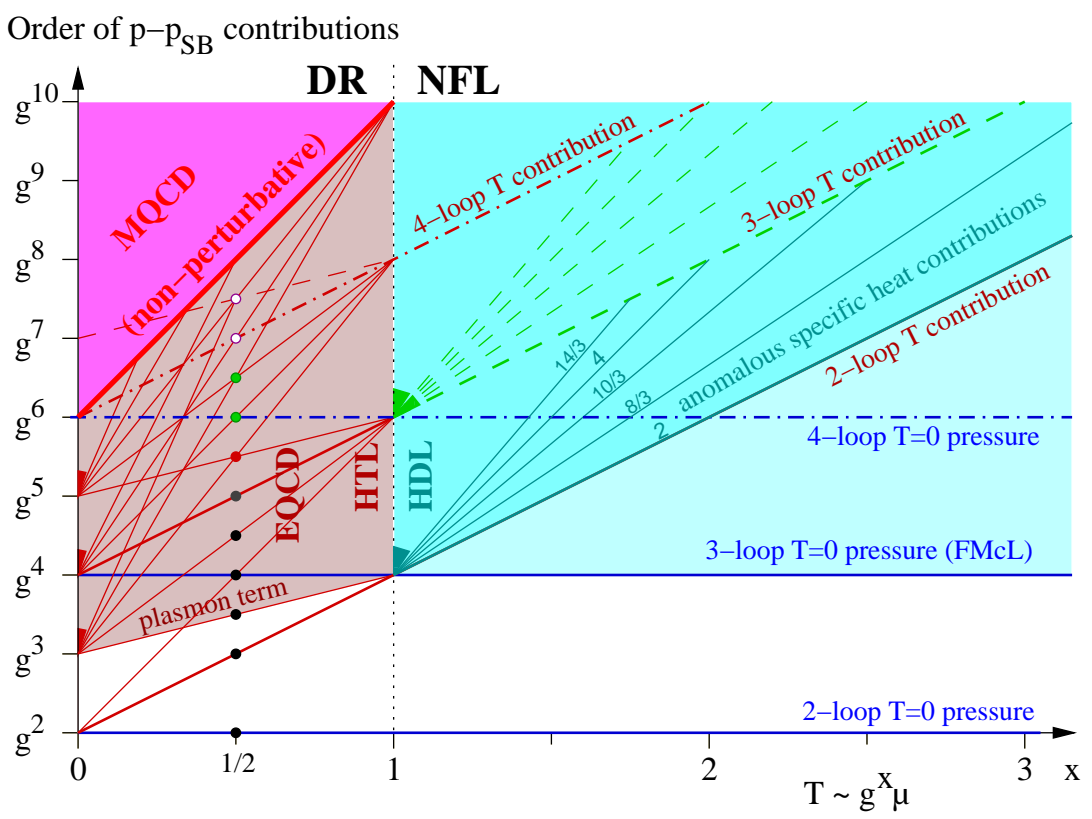

FIG. 17: The structure of the weak-coupling expansion of the interaction pressure $p-p_{\mathrm{SB}}$ at parametrically small $T / \mu$ as a function of the power $x$ in $T \sim g^{x} \mu$. At $T \sim \mu$, i.e. $x=0$, the expansion involves orders 2,3 , $4, \ldots$ in $g$ (logarithms in $g$ are not made explicit); at $T \sim g^{1 / 2} \mu$ where dimensional reduction overlaps with HTL/HDL resummation, the series in $g$ involves powers $2,3, \frac{7}{2}, 4, \frac{9}{2}, \ldots$; at $T \sim g \mu$, where dimensional reduction ceases to be applicable, the expansion is again in even powers of $g$ (and logs) with coefficients that at even smaller temperatures can be expanded in a series involving fractional powers of $T$ (beginning with $\left.2, \frac{8}{3}, \frac{10}{3}, 4, \frac{14}{3}, \ldots\right)$ and corresponding powers $2+2 x, 2+\frac{8}{3} x, \ldots$ of $g$. While subleading in the pressure, the latter contributions give the leading-order "anomalous" (non-Fermi-liquid) contributions to the interaction part of the entropy and specific heat at low temperature. Existing results for the various contributions are represented by full lines, as-yet undetermined contributions by dashed and dash-dotted lines. The nonperturbative barrier from the scale of magnetostatic confinement (magnetic screening mass) is indicated by the thick red line marked "non-perturbative". The region below it and up to $x=1$ is the regime of electrostatic QCD (EQCD), while for $x \geq 1$ the relevant effective theory is given by non-static hard dense loops (HDL).

higher-order terms for $x>0$. These arise from the expansion of the third power of the Debye mass parameter in powers of $T / \mu$, and, for subsequent terms in the dimensional reduction result, also from the expansion of the special functions $\aleph(n, z)$. Because both the Debye mass and the $\aleph$ functions can be expanded in even powers of $T / \mu$, the lines emanating from their starting points at $x=0$ come with slopes differing by two units. The terms proportional to $g^{3}$ and $g^{5}$ at $x=0$ involve a single overall power of $T$, so the lines emanating from these have slopes $1,3,5, \ldots$, whereas the term proportional to $g^{4}$ ( or $g^{4} \ln g$ ) has also $T$-independent parts and thus gives rise to lines with slopes $0,2,4, \ldots$ In Eq. (2.6) we have seen how this gives rise to a new series in $g$ at $x=1 / 2$, and Fig. [17 illustrates how the individual terms of order $2,3, \frac{7}{2}, 4, \frac{9}{2}, \ldots$ are produced from the various coefficients of the expansion at $x=0$.

Moving on to the border of applicability of the dimensional reduction results, $x=1$, we see that all lines converge to points corresponding to an expansion in even powers of $g$ (and also involving $\ln g$ ). As noted before, for $x \geq 1$ the relevant effective theory is the one given by non-static hard dense loops. Their resummation is necessary to obtain the classic Freedman-McLerran (FMcL) result to order $g^{4}$ (again accompanied by a logarithmic term) at $T=0$ as well as the leading thermal corrections to the interaction pressure. In a low- $T$ expansion these $T$-dependent terms 
start with a contribution of order $g^{2} T^{2} \mu^{2} \ln (T / g \mu)$ and then involve fractional powers $T^{8 / 3}, T^{10 / 3}$, $T^{4} \ln T, T^{14 / 3}, \ldots$ such that the corresponding lines in Fig. 17 (labeled by the exponent of $T$ ) meet at $x=1$ and effective order $g^{4}$. At this point, the leading $T$-dependent contributions are of the same order as the three-loop $T=0$ (FMcL) pressure contribution and remain more important than the undetermined four-loop $T=0$ term even for parametrically lower temperatures as long

as $x<2$ (i.e. $T \gg g^{2} \mu$ ). For the entropy and specific heat, for which the zero-temperature contribution to the pressure drops out, these $T$-dependent terms represent the leading interaction contributions down to arbitrarily low temperatures. The $T \ln T$ behavior of the entropy (as well as of the specific heat) is characterized by "anomalous" non-Fermi-liquid behavior, caused by the only weakly (dynamically) screened quasi-static magnetic interactions with an effective frequencydependent screening mass, displayed in Eq. (2.9).

As suggested by Fig. 177and shown in detail in the previous section, the HDL-resummed thermal pressure contributions responsible for the non-Fermi-liquid behavior at $T \ll g \mu$ match smoothly to the perturbative effects at $T \gg g \mu$ described by EQCD. As the temperature is increased, electrostatic screening replaces dynamical magnetic screening as the dominant collective phenomenon also in the $T$-dependent contributions. For $T$ parametrically larger than $g \mu($ i.e. $x<1)$ the resummation of HDL self energies needs to be trivially extended to HTL self energies to avoid accuracy loss. When added to the zero temperature $\mathcal{O}\left(g^{4}\right)$ result, this gives an expression that gives the pressure for all temperatures and chemical potentials up to an error of order $g^{\min (4+2 x, 6)}$ (or $g^{4+x}$ throughout in the case of the entropy, for which the unknown four-loop $\mathrm{T}=0$ pressure drops out).

From the "flow" of the various perturbative contributions as a function of $x$ in Fig. 17] one notices that a single expression aiming to be valid both for $x>1$ and $x<1$ needs to keep track of contributions which are perhaps higher-order and irrelevant in some region but essential in another. The novel approach we have presented here does so by resumming the complete oneloop gluon self-energy in all IR sensitive graphs while treating the infrared-safe 2GI diagrams perturbatively. To the extent that we have worked it out, this procedure covers both $x>1$ and $x<1$ with an error of order $g^{\min (5+x, 6)}$ which improves over the HTL/HDL result in the region $x<1$ by including the contributions of all relevant three-loop graphs. A drawback compared to the HTL/HDL resummation schemes is however that the resummation of the complete gluon self-energy leads to gauge-dependent higher-order contributions whose unphysical nature is highlighted by the appearance of spacelike poles in the logarithmic resummation integrand with momenta $\sim g^{2} T$ and also of an unphysical damping constant (with an incorrect sign) $\propto g^{2} T$. For our expression for the pressure, the effect of these problems is, however, only of the order of the nonperturbative MQCD contributions, i.e. $g^{6}$, so it has not hindered us from confirming and thus validating the results obtained through dimensional reduction or the HTL/HDL approach.

\section{CONCLUSIONS AND OUTLOOK}

In this paper, we have constructed a novel resummation scheme designed to reproduce the weak coupling expansion of the QCD pressure up to order $g^{4}$ on the entire $\mu-T$ plane. We have used it to provide an independent check of practically all existing perturbative results. In particular, we have performed the first explicit test on the validity of dimensional reduction for values of $\mu / T$ far beyond the capability of present-day lattice techniques, thus verifying that dimensionally reduced effective theories provide a solid description of the perturbative physics up to in principle arbitrarily large values of $\mu / T$ as long as $\pi T>m_{\mathrm{D}}$. At temperatures parametrically smaller than the chemical potential, we have on the other hand reproduced numerically all the results of the HTL/HDL resummation schemes, verifying their validity and highlighting the smooth transition taking place in the perturbative expansion of the pressure as one moves from the region of dimensional reduction 
towards the zero-temperature limit.

Based on our numerical results from Section IV the dimensional reduction result for the QCD pressure appears to be provide a remarkably good approximation for this quantity down to the point where the $T$-dependent contribution to the interaction pressure, $\delta p$, ceases to be negative (cf. Figs. [7f) which happens at $T \approx 0.2 m_{\mathrm{D}}$. Since the dimensional reduction result to order $g^{6} \ln g$ combined with optimized choices of the renormalization scale has turned out to agree rather well with lattice results, both at zero chemical potential [7, 41] and for $\mu \sim T$ [8, 42], our present findings in fact suggest a remarkably wide practical range of applicability for the dimensional reduction method and results.

Progressing down on the temperature axis to $T \lesssim 0.2 m_{\mathrm{D}}$, one eventually has to switch to the nonstatic resummation schemes provided either by our new approach or by the calculationally much simpler HTL resummation of Eq. (2.15). At such low temperatures, the pressure can - up to but not including order $g^{6}$ - be approximated by the Freedman-McLerran result plus positive contributions from the Stefan-Boltzmann terms as well as the interaction pressure $\delta p$. The latter of these is the source of the non-Fermi-liquid behavior of the entropy and specific heat.

While we believe to have thoroughly clarified the nature of perturbative expansions of the pressure in different regimes of the $\mu-T$ plane, our new approach is, as of today, yet to produce results for the pressure beyond what has already been achieved through either dimensional reduction at $x<1$, the HTL/HDL resummation schemes at $x \geq 1$ or the Freedman-McLerran result at $T=0$. Its present relative error of order $g^{\min (5+x, 6)}$ can in principle be reduced through the inclusion of the two-loop gluon polarization tensor into the resummation of the ring diagrams and in addition by taking the contributions of non-static modes into account in the multiple sums of Fig. 2.bd. For the pressure, this would bring the accuracy of our new approach up to the one currently achieved by dimensional reduction calculations (excluding the already known $\mathcal{O}\left(g^{6} \ln g T^{2}\left(T^{2}+\mu^{2}\right)\right.$ ) term), so that the error, up to logarithms, would be uniformly (for all values of $x$ ) of order $g^{6} \ln g$, corresponding to the line marked "4-loop $T=0$ pressure" in Fig. 17. This would then unify all existing perturbative results for the pressure of QCD, while for the entropy it would moreover lead to genuinely new results. Apart from increasing e.g. the accuracy of the entropy result at $x=1 / 2$ to order $g^{13 / 2}$ (green open dots in Fig. 17) ${ }^{15}$, it would push the error in the $T$-dependent part of the pressure up to the line denoted in Fig. 17] by "4-loop T contribution" and thus, for $x>1$, include the so far unknown order $g^{4} \mu^{2} T$ corrections to the non-Fermi-liquid terms in the entropy and the specific heat. Considering the difficulties caused by the gauge-dependent parts of the gluon self-energy, it seems that such an extension should probably aim at keeping only gaugeindependent contributions such as HTL self energies in the ring diagrams and treating corrections to those self-energies in a perturbative manner. Work towards this goal is currently in progress.

\section{Acknowledgments}

We are grateful to Mikko Laine, York Schröder, and Larry Yaffe for their helpful comments and suggestions and to Dirk Rischke for discussions on color superconductivity. This work has been partially supported by the Austrian Science Foundation FWF, project no. P16387-N08 and the Academy of Finland, project no. 109720.

15 The highest purely perturbatively calculable order at $x=1 / 2$ is $g^{15 / 2}$ which would require a calculation of the contributions of order $g^{6} \mu^{2} T^{2}$ and $g^{7} \mu^{3} T$ for the pressure. 


\section{APPENDIX A: DIMENSIONAL REDUCTION RESULT AT FINITE $T$ AND $\mu$}

To order $g^{5}$ and simplified by assuming equal chemical potentials, the result of dimensional reduction for the QCD pressure at finite $T$ and $\mu$, obtained in Ref. [8], reads

$$
\begin{aligned}
& \frac{p_{\mathrm{DR}}(T, \mu)}{T^{4}}=\frac{\pi^{2}}{45}\left\{d_{A}+\left(\frac{7}{4}+30 \bar{\mu}^{2}+60 \bar{\mu}^{4}\right) d_{F}\right\} \\
& -g^{2} \frac{d_{A}}{144}\left\{C_{A}+\frac{T_{F}}{2}\left(1+12 \bar{\mu}^{2}\right)\left(5+12 \bar{\mu}^{2}\right)\right\} \\
& +\frac{g^{3}}{(4 \pi)} \frac{d_{A}}{3}\left\{\frac{1}{3}\left(C_{A}+T_{F}\left(1+12 \bar{\mu}^{2}\right)\right)\right\}^{3 / 2} \\
& +\frac{g^{4}}{(4 \pi)^{2}} \frac{d_{A}}{144}\left\{48 C_{A}\left(C_{A}+T_{F}\left(1+12 \bar{\mu}^{2}\right)\right) \ln \left[\left(C_{A}+T_{F}\left(1+12 \bar{\mu}^{2}\right)\right)^{1 / 2} g\right]\right. \\
& -C_{A}^{2}\left(\frac{22}{3} \ln \frac{\bar{\Lambda}}{4 \pi T}+\frac{64}{5}+24 \ln 12 \pi^{2}-4 \gamma+\frac{38}{3} \frac{\zeta^{\prime}(-3)}{\zeta(-3)}-\frac{148}{3} \frac{\zeta^{\prime}(-1)}{\zeta(-1)}\right) \\
& -C_{A} T_{F}\left(\left(\frac{47}{3}+264 \bar{\mu}^{2}+528 \bar{\mu}^{4}\right) \ln \frac{\bar{\Lambda}}{4 \pi T}+\frac{1759}{60}+8 \gamma+24\left(1+12 \bar{\mu}^{2}\right) \ln \left(12 \pi^{2}\right)\right. \\
& +2(161-48 \gamma) \bar{\mu}^{2}-644 \bar{\mu}^{4}-\frac{268}{15} \frac{\zeta^{\prime}(-3)}{\zeta(-3)}-\frac{4}{3}\left(11+156 \bar{\mu}^{2}\right) \frac{\zeta^{\prime}(-1)}{\zeta(-1)} \\
& \left.-24\left[52 \aleph(3, z)+144 i \bar{\mu} \aleph(2, z)+\left(5-92 \bar{\mu}^{2}\right) \aleph(1, z)+4 i \bar{\mu} \aleph(0, z)\right]\right) \\
& +C_{F} T_{F}\left(\frac{3}{4}\left(1+4 \bar{\mu}^{2}\right)\left(35+332 \bar{\mu}^{2}\right)-24\left(1-4 \bar{\mu}^{2}\right) \frac{\zeta^{\prime}(-1)}{\zeta(-1)}\right. \\
& \left.-144\left[12 i \bar{\mu} \aleph(2, z)-2\left(1+8 \bar{\mu}^{2}\right) \aleph(1, z)-i \bar{\mu}\left(1+4 \bar{\mu}^{2}\right) \aleph(0, z)\right]\right) \\
& +T_{F}^{2}\left(\frac{4}{3}\left(1+12 \bar{\mu}^{2}\right)\left(5+12 \bar{\mu}^{2}\right) \ln \frac{\bar{\Lambda}}{4 \pi T}+\frac{1}{3}+4 \gamma+8(7+12 \gamma) \bar{\mu}^{2}\right. \\
& +16(43+36 \gamma) \bar{\mu}^{4}-\frac{64}{15} \frac{\zeta^{\prime}(-3)}{\zeta(-3)}-\frac{32}{3}\left(1+12 \bar{\mu}^{2}\right) \frac{\zeta^{\prime}(-1)}{\zeta(-1)} \\
& -96\left[8 \aleph(3, z)+12 i \bar{\mu} \aleph(2, z)-2\left(1+8 \bar{\mu}^{2}\right) \aleph(1, z)\right. \\
& \left.\left.\left.-i \bar{\mu}\left(1+12 \bar{\mu}^{2}\right) \aleph(0, z)+3 \aleph(3,2 z)+12 i \bar{\mu} \aleph(2,2 z)-12 \bar{\mu}^{2} \aleph(1,2 z)\right]\right)\right\} \\
& +\frac{g^{5}}{(4 \pi)^{3}} d_{A} \sqrt{\frac{1}{3}\left(C_{A}+T_{F}\left(1+12 \bar{\mu}^{2}\right)\right)}\left\{-C_{F} T_{F}\left(1+12 \bar{\mu}^{2}\right)\right. \\
& +C_{A}^{2}\left(\frac{11}{9} \ln \frac{\bar{\Lambda}}{4 \pi T}-\frac{247}{72}+\frac{11}{6} \ln 2+\frac{11}{9} \gamma-\frac{\pi^{2}}{6}\right) \\
& +C_{A} T_{F}\left(\left(\frac{7}{9}+\frac{44}{3} \bar{\mu}^{2}\right) \ln \frac{\bar{\Lambda}}{4 \pi T}+\frac{1}{2}+\frac{11}{9} \gamma+\frac{22}{3}(1+2 \gamma) \bar{\mu}^{2}+\frac{2}{9} \aleph(z)\right) \\
& \left.-T_{F}^{2}\left(\left(\frac{4}{9}+\frac{16}{3} \bar{\mu}^{2}\right) \ln \frac{\bar{\Lambda}}{4 \pi T}-\frac{2}{9}-\frac{8}{3} \bar{\mu}^{2}-\frac{2}{9}\left(1+12 \bar{\mu}^{2}\right) \aleph(z)\right)\right\}+\mathcal{O}\left(g^{6} \ln g\right) \text {. }
\end{aligned}
$$




\section{APPENDIX B: THE ANALYTIC VALUES OF VARIOUS PARTS OF THE PRESSURE}

In this Appendix, we collect some calculational details on the different pieces of the analytical part of the pressure, $p_{\mathrm{anl}}$, as defined in (3.17). The sum of the 2GI diagrams was already given in Eq. (3.1) and the piece $p_{\mathrm{b}}$ in Eq. (3.16).

\section{The Vac-Vac and Vac-Mat diagrams}

The evaluation of the two special diagrams of Fig. 2, dubbed Vac-Vac and Vac-Mat based on their self energy insertions, is relatively straightforward. Inserting the form of $\left.\Pi_{\mu \nu}^{a b}(P)\right|_{\text {vac }}$ from Eq. (C4) into the Feynman gauge expressions of the graphs, contracting all Lorentz and color indices and taking into account the symmetry factors $1 / 4$ and $1 / 2$, respectively, we readily obtain

$$
\begin{aligned}
p_{\mathrm{VV}} & =\frac{1}{4}(d-1) d_{A} \tilde{A}^{2} g^{4} \Lambda^{4 \epsilon} \mathcal{I}_{2 \epsilon}^{0} \\
& =\frac{d_{A}\left(5 C_{A}-4 T_{F}\right)^{2}}{4320(4 \pi)^{2}} g^{4} T^{4}\left(\frac{1}{\epsilon}+6 \ln \frac{\bar{\Lambda}}{4 \pi T}+\frac{32}{3}+6 \frac{\zeta^{\prime}(-3)}{\zeta(-3)}+\frac{4 C_{A}}{5 C_{A}-4 T_{F}}\right), \\
p_{\mathrm{VM}} & =-2 p_{\mathrm{VV}}-\frac{1}{2} d_{A} \tilde{A} g^{4} \Lambda^{2 \epsilon}\left\{\left(C_{A}(d-2)^{2} \mathcal{I}_{1}^{0}-4 T_{F}(d-2) \widetilde{\mathcal{I}}_{1}^{0}\right) \mathcal{I}_{1+\epsilon}^{0}\right. \\
& \left.-(5-3 \epsilon) C_{A} \oint_{P Q} \frac{1}{\left(P^{2}\right)^{\epsilon} Q^{2}(P+Q)^{2}}+2(d-2) T_{F} \oint_{P\{Q\}} \frac{1}{\left(P^{2}\right)^{\epsilon} Q^{2}(P+Q)^{2}}\right\},
\end{aligned}
$$

where we in the latter case have two two-loop sum-integrals to evaluate. These can be taken care of using methods developed in Refs. [4, 8], which leads to the final result

$$
\begin{aligned}
p_{\mathrm{VM}} & =-2 p_{\mathrm{VV}}+\frac{d_{A} g^{4} T^{4}}{4320(4 \pi)^{2}}\left\{\frac{\left(5 C_{A}-4 T_{F}\right)\left(10 C_{A}-\left(29+360 \bar{\mu}^{2}+720 \bar{\mu}^{4}\right) T_{F}\right)}{\epsilon}\right. \\
& +100 C_{A}^{2}\left(3 \ln \frac{\bar{\Lambda}}{4 \pi T}+\frac{463}{60}-\frac{\zeta^{\prime}(-1)}{\zeta(-1)}+4 \frac{\zeta^{\prime}(-3)}{\zeta(-3)}\right) \\
& +30 C_{A} T_{F}\left(-\left(37+360 \bar{\mu}^{2}+720 \bar{\mu}^{4}\right) \ln \frac{\bar{\Lambda}}{4 \pi T}-\frac{8971}{180}-\frac{32}{3} \frac{\zeta^{\prime}(-1)}{\zeta(-1)}-\frac{40}{3} \frac{\zeta^{\prime}(-3)}{\zeta(-3)}\right. \\
& \left.-2\left(167+80 \frac{\zeta^{\prime}(-1)}{\zeta(-1)}\right) \bar{\mu}^{2}-668 \bar{\mu}^{4}-240\left(1+4 \bar{\mu}^{2}\right) \aleph(1, z)-480 \aleph(3, z)\right) \\
& +24 T_{F}^{2}\left(\left(29+360 \bar{\mu}^{2}+720 \bar{\mu}^{4}\right) \ln \frac{\bar{\Lambda}}{4 \pi T}+\frac{1003}{36}+\frac{40}{3} \frac{\zeta^{\prime}(-1)}{\zeta(-1)}+\frac{8}{3} \frac{\zeta^{\prime}(-3)}{\zeta(-3)}\right. \\
& \left.\left.+10\left(31+16 \frac{\zeta^{\prime}(-1)}{\zeta(-1)}\right) \bar{\mu}^{2}+620 \bar{\mu}^{4}+240\left(1+4 \bar{\mu}^{2}\right) \aleph(1, z)+480 \aleph(3, z)\right)\right\} .
\end{aligned}
$$

\section{The ordinary ring sum}

The UV subtraction term $p_{\text {ring }}^{\mathrm{UV}}$ of the ordinary ring sum, defined in Eq. (3.8), is straightforward to evaluate and produces the result

$$
\begin{aligned}
p_{\text {ring }}^{\mathrm{UV}} & =(3+4 \epsilon) d_{A} C_{A}^{2} g^{4}\left(\mathcal{I}_{1}^{0}\right)^{2} \mathcal{I}_{2}^{0} \\
& =\frac{d_{A} C_{A}^{2}}{48(4 \pi)^{2}} g^{4} T^{4}\left(\frac{1}{\epsilon}+6 \ln \frac{\bar{\Lambda}}{4 \pi T}+\frac{16}{3}+2 \gamma+4 \frac{\zeta^{\prime}(-1)}{\zeta(-1)}\right)+\mathcal{O}(\epsilon) .
\end{aligned}
$$


With the IR regulating term $p_{\text {ring }}^{\mathrm{IR}}$, we find it most convenient to first transform the Matsubara sum into a contour integral in the standard way. After the term proportional to a bosonic distribution function is analytically continued to Minkowski space (see Appendix D), this leads to the result

$$
\begin{aligned}
p_{\text {ring }}^{\mathrm{IR}} & =\frac{d_{A} C_{A}^{2} g^{4} T^{4}}{48}\left(-\mathcal{I}_{2}^{0}+\Lambda^{2 \epsilon} \int \frac{d^{3-2 \epsilon} p}{(2 \pi)^{3-2 \epsilon}}\left\{\frac{1}{2 \pi i} \int_{-i \infty}^{i \infty} d p_{0} \frac{1}{\left(p_{0}^{2}-p^{2}-m^{2}\right)^{2}}\right.\right. \\
& \left.\left.-\frac{1}{\pi} \int_{0}^{\infty} d p_{0} n_{b}\left(p_{0}\right) \operatorname{Im}\left[\frac{1}{\left(\left(p_{0}+i \epsilon\right)^{2}-p^{2}-m^{2}\right)^{2}}\right]\right\}\right) .
\end{aligned}
$$

The sum of the first two terms is evaluated with standard methods and gives

$$
-\mathcal{I}_{2}^{0}+\frac{\Lambda^{2 \epsilon}}{2 \pi i} \int \frac{d^{3-2 \epsilon} p}{(2 \pi)^{3-2 \epsilon}} \int_{-i \infty}^{i \infty} d p_{0} \frac{1}{\left(p_{0}^{2}-p^{2}-m^{2}\right)^{2}}=-\frac{2}{(4 \pi)^{2}}\left(\ln \frac{m}{4 \pi T}+\gamma\right),
$$

while the third term (where one can set $\epsilon=0$ ) is left in its present form until Appendix D where it is seen to explicitly cancel with certain parts of $p_{\text {ring }}^{\text {finite }}$. Modifications to $p_{\text {ring }}^{\mathrm{IR}}$ induced by a magnetic mass are discussed after Eq. (D5).

\section{Small and large $\mu / T$ limits of $p_{\text {anl }}$}

For convenience, we provide here formulae for various limits of the function $p_{\text {anl }}$ of Eq. (3.20), derived using the results of Appendix D of Ref. [8] for the behavior of the $\aleph$ functions. First, in the limit $\mu \rightarrow 0$ we obtain through an expansion in $\bar{\mu} \equiv \frac{\mu}{2 \pi T}$

$$
\begin{aligned}
p_{\text {anl }} \underset{\mu \rightarrow 0}{\longrightarrow} & \pi^{2} d_{A} T^{4}\left\{\frac{1}{45}\left\{1+\frac{d_{F}}{d_{A}}\left(\frac{7}{4}+30 \bar{\mu}^{2}\right)\right\}-\frac{g^{2}}{9(4 \pi)^{2}}\left\{C_{A}+\frac{T_{F}}{2}\left(1+72 \bar{\mu}^{2}\right)\right\}\right. \\
& +\frac{g^{4}}{27(4 \pi)^{4}}\left\{-C_{A}^{2}\left(22 \ln \frac{\bar{\Lambda}}{4 \pi T}+63-18 \gamma+110 \frac{\zeta^{\prime}(-1)}{\zeta(-1)}-70 \frac{\zeta^{\prime}(-3)}{\zeta(-3)}\right)\right. \\
& -\quad C_{A} T_{F}\left(\left(47+792 \bar{\mu}^{2}\right) \ln \frac{\bar{\Lambda}}{4 \pi T}+\frac{2391}{20}+70 \frac{\zeta^{\prime}(-1)}{\zeta(-1)}-23 \frac{\zeta^{\prime}(-3)}{\zeta(-3)}-\frac{297}{5} \ln 2\right. \\
& \left.+6(235+352 \ln 2+132 \gamma) \bar{\mu}^{2}\right) \\
& +\quad T_{F}^{2}\left(4\left(5+72 \bar{\mu}^{2}\right) \ln \frac{\bar{\Lambda}}{4 \pi T}+\frac{99}{5}-\frac{108}{5} \ln 2+40 \frac{\zeta^{\prime}(-1)}{\zeta(-1)}-20 \frac{\zeta^{\prime}(-3)}{\zeta(-3)}\right. \\
& \left.+24(11+32 \ln 2+12 \gamma) \bar{\mu}^{2}\right) \\
& \left.\left.+\frac{9}{4} C_{F} T_{F}\left(35-32 \ln 2+8(35+16 \ln 2) \bar{\mu}^{2}\right)\right\}+\mathcal{O}\left(\bar{\mu}^{4}\right)\right\}
\end{aligned}
$$


which contains e.g. the $C_{F} T_{F}$ dependent part of the linear quark number susceptibilities found in Ref. [4]]. Taking the opposite limit, $T \rightarrow 0$, we on the other hand get

$$
\begin{aligned}
p_{\text {anl }} \underset{T \rightarrow 0}{\longrightarrow} & \frac{d_{A} \mu^{4}}{(4 \pi)^{2}}\left\{4\left(\frac{d_{F}}{3 d_{A}}-2 T_{F} \frac{g^{2}}{(4 \pi)^{2}}\right)\left(1+\frac{1}{2 \bar{\mu}^{2}}\right)\right. \\
& -\frac{8}{3} \frac{g^{4}}{(4 \pi)^{4}}\left\{1 1 C _ { A } T _ { F } \left(2 \ln \frac{\bar{\Lambda}}{2 \mu}+\frac{142}{33}\right.\right. \\
& \left.+\quad\left[\ln \frac{\bar{\Lambda}}{4 \pi T}-\frac{1}{3} \ln \bar{\mu}+\frac{395}{132}-\frac{48}{11} \ln 2+\frac{2}{3} \frac{\zeta^{\prime}(-1)}{\zeta(-1)}\right] \bar{\mu}^{-2}\right) \\
& -\quad 4 T_{F}^{2}\left(2 \ln \frac{\bar{\Lambda}}{2 \mu}+\frac{11}{3}+\left[\ln \frac{\bar{\Lambda}}{4 \pi T}-\frac{1}{3} \ln \bar{\mu}+\frac{19}{12}+\frac{2}{3} \frac{\zeta^{\prime}(-1)}{\zeta(-1)}\right] \bar{\mu}^{-2}\right) \\
& \left.\left.-\quad C_{F} T_{F}\left(\frac{51}{2}+\left[4 \ln \bar{\mu}+\frac{35}{4}+4 \frac{\zeta^{\prime}(-1)}{\zeta(-1)}\right] \bar{\mu}^{-2}\right)\right\}+\mathcal{O}\left(\bar{\mu}^{-4} \ln \bar{\mu}\right)\right\} .
\end{aligned}
$$

This yields an entirely finite expression at $T=0$, where the scale dependence of the result naturally coincides with that of Eq. (2.7).

\section{APPENDIX C: PROPERTIES OF THE ONE-LOOP GLUON POLARIZATION TENSOR}

For reference, we note that the one-loop gluon polarization tensor in the Feynman gauge can be written in the form

$$
\begin{aligned}
\Pi_{\mu \nu}^{a b}(P) & =g^{2} \delta^{a b}\left\{C _ { A } \left((d-2) \mathcal{I}_{1}^{0} \delta_{\mu \nu}+2\left(P_{\mu} P_{\nu}-P^{2} \delta_{\mu \nu}\right) \Pi(P)\right.\right. \\
& \left.-\frac{d-2}{2} \sum_{Q} \frac{(2 Q-P)_{\mu}(2 Q-P)_{\nu}}{Q^{2}(Q-P)^{2}}\right) \\
& \left.-2 T_{F}\left(2 \widetilde{\mathcal{I}}_{1}^{0} \delta_{\mu \nu}+\left(P_{\mu} P_{\nu}-P^{2} \delta_{\mu \nu}\right) \Pi_{\mathrm{f}}(P)-\sum_{\{Q\}} \frac{(2 Q-P)_{\mu}(2 Q-P)_{\nu}}{Q^{2}(Q-P)^{2}}\right)\right\},
\end{aligned}
$$

where we have, as usual, defined

$$
\begin{aligned}
\Pi(P) & \equiv \sum_{Q} \frac{1}{Q^{2}(Q-P)^{2}}, \\
\Pi_{\mathrm{f}}(P) & \equiv \sum_{\{Q\}} \frac{1}{Q^{2}(Q-P)^{2}} .
\end{aligned}
$$

The vacuum $(T, \mu \rightarrow 0)$ limit of the above formula is uniquely determined by replacing the sumintegrals by $4-2 \epsilon$-dimensional integrals which produces

$$
\left.\Pi_{\mu \nu}^{a b}(P)\right|_{\mathrm{vac}}=\tilde{A} g^{2} \delta^{a b}\left(\frac{\Lambda^{2}}{P^{2}}\right)^{\epsilon}\left(P_{\mu} P_{\nu}-P^{2} \delta_{\mu \nu}\right)
$$

with

$$
\tilde{A} \equiv \frac{\left((5 / 2+\epsilon) C_{A}-2 T_{F}\right)}{24 \pi^{2}}\left(\frac{1}{\epsilon}-\gamma+\ln (4 \pi)+\frac{5}{3}+\mathcal{O}(\epsilon)\right)
$$

As all our analytic formulae, the above expressions have been given in Euclidean space. For the numerical evaluation of $p_{\text {ring }}^{\text {safe }}$ it is, however, convenient to also reproduce the known formulae for 
the transverse and longitudinal pieces of the vacuum-subtracted self energy in Minkowski space. Following Ref. [45], we obtain

$$
\begin{aligned}
& \Pi_{\mathrm{L}}\left(q_{0}, q\right)=-g^{2} \frac{Q^{2}}{q^{2}}\left(2 T_{F} H_{f}+C_{A} H_{b}\right), \\
& \Pi_{\mathrm{T}}\left(q_{0}, q\right)=\frac{g^{2}}{2}\left(\frac{Q^{2}}{q^{2}}\left(2 T_{F} H_{f}+C_{A} H_{b}\right)+\left(2 T_{F} G_{f}+C_{A} G_{b}\right)\right),
\end{aligned}
$$

where the different functions read

$$
\begin{aligned}
G_{f} & =\frac{1}{2 \pi^{2}} \int_{0}^{\infty} d k n_{f}(k)\left(4 k-\frac{q^{2}-q_{0}^{2}}{2 q} L_{1}\right), \\
H_{f} & =\frac{1}{2 \pi^{2}} \int_{0}^{\infty} d k n_{f}(k)\left(2 k-\frac{q^{2}-q_{0}^{2}-4 k^{2}}{4 q} L_{1}-q_{0} k L_{2}\right), \\
G_{b} & =\frac{1}{2 \pi^{2}} \int_{0}^{\infty} d k n_{b}(k)\left(4 k-\frac{5}{4} \frac{q^{2}-q_{0}^{2}}{q} L_{1}\right), \\
H_{b} & =\frac{1}{2 \pi^{2}} \int_{0}^{\infty} d k n_{b}(k)\left(2 k-\frac{2 q^{2}-q_{0}^{2}-4 k^{2}}{4 q} L_{1}-q_{0} k L_{2}\right),
\end{aligned}
$$

with

$$
\begin{aligned}
& L_{1}=\log \left(\frac{2 k+q-q_{0}}{2 k-q-q_{0}}\right)-\log \left(\frac{2 k-q+q_{0}}{2 k+q+q_{0}}\right), \\
& L_{2}=\log \left(\frac{2 k+q-q_{0}}{2 k-q-q_{0}}\right)-2 \log \left(\frac{-q+q_{0}}{q+q_{0}}\right)+\log \left(\frac{2 k-q+q_{0}}{2 k+q+q_{0}}\right) .
\end{aligned}
$$

These expressions are valid for all complex $q_{0}$ in a rotation from Euclidean space $q_{0}=i \omega$ to Minkowski space $q_{0}=\omega+i \epsilon$ with $\epsilon>0$. For the analytic continuation into the region with $\epsilon<0$, see Ref. [43].

The corresponding HTL and HDL expressions for the above functions can be extracted by demanding that the external momenta $q_{0}$ and $q$ be small compared to the temperature $T$ and/or the chemical potential $\mu$. This amounts to expanding the integrand in inverse powers of $k$ to leading order, since the main contribution is expected to come from large loop momenta $k$. The relevant physical scale is given by the Debye mass $m_{\mathrm{D}}$. In this limit, the equations (C8) to (C11) reduce to

$$
\begin{aligned}
G_{f}^{\mathrm{HTL}} & =\frac{T^{2}}{6}+\frac{\mu^{2}}{2 \pi^{2}}, \\
H_{f}^{\mathrm{HTL}} & =\left(\frac{T^{2}}{6}+\frac{\mu^{2}}{2 \pi^{2}}\right)\left\{1+\frac{q_{0}}{2 q} \log \left(\frac{-q+q_{0}}{q+q_{0}}\right)\right\}, \\
G_{b}^{\mathrm{HTL}} & =\frac{T^{2}}{3}, \\
H_{b}^{\mathrm{HTL}} & =\frac{T^{2}}{3}\left\{1+\frac{q_{0}}{2 q} \log \left(\frac{-q+q_{0}}{q+q_{0}}\right)\right\} .
\end{aligned}
$$

The HDL expressions are obtained by setting $T=0$ here which makes both $G_{b}^{\mathrm{HDL}}$ and $H_{b}^{\mathrm{HDL}}$ vanish.

\section{APPENDIX D: NUMERICAL EVALUATION OF $p_{\text {ring }}^{\text {finite }}$}

The numerical evaluation of the sum-integrals introduced in Sec. III is performed by converting the sums over Matsubara frequencies into contour integrals where the integrand is multiplied by 
$\cot \left(\omega_{n} / 2 T\right)$ and the contour encircles the poles of this function (which lie on the real axis at $\omega_{n}=2 \pi n T$ and have the residue $\left.2 T\right)$. We then change the integration variable from $q_{0}=i \omega_{n}$, with real $\omega_{n}$, to $q_{0}+i \epsilon$, with real $q_{0}$ and $\epsilon>0$, deforming the integration contour in the usual way (for more details on this, see e.g. Ref. [33]). Through this procedure, we obtain the generic result

$$
\begin{aligned}
T \sum_{\omega_{n}=2 \pi n T}^{n \in Z} f\left(i \omega_{n}\right) & =\int_{0}^{\infty} \frac{d q_{0}}{\pi} \operatorname{coth}\left(\frac{q_{0}}{2 T}\right) \frac{-i}{2}\left\{f\left(q_{0}+i \epsilon\right)-f^{*}\left(q_{0}+i \epsilon\right)\right\} \\
& =\int_{0}^{\infty} \frac{d q_{0}}{\pi}\left(1+2 n_{b}\right) \operatorname{Im} f\left(q_{0}+i \epsilon\right)
\end{aligned}
$$

where we have used the identity $\operatorname{coth} \frac{q_{0}}{2 T}=1+2 n_{b}$. This is valid for any function $f$ satisfying $f\left(q_{0}+i \epsilon\right)=f^{*}\left(q_{0}-i \epsilon\right)=f\left(-q_{0}-i \epsilon\right)=f^{*}\left(-q_{0}+i \epsilon\right)$, provided that the great arc contribution to the integral vanishes and there are no other poles or branch cuts between the Euclidean and Minkowskian axes.

After these standard manipulations, we split the above integral into the UV safe " $n_{b}$ part" which we calculate in Minkowski space, i.e. along the real $q_{0}$ axis, and the "non- $n_{b}$ part" that we rotate back to Euclidean space ${ }^{16}$ before integration through

$$
\int_{0}^{\infty} \frac{d q_{0}}{\pi} \operatorname{Im} f\left(q_{0}+i \epsilon\right) \rightarrow \int_{0}^{\infty} \frac{d \omega}{\pi} \operatorname{Re} f(i \omega)
$$

In this way, we take full advantage of the UV cutoff properties of $n_{b}$ in Minkowski space while the possibly UV problematic non- $n_{b}$ parts are treated respecting the Euclidean symmetry. Further discussion on the reasons for this separation can be found from Refs. [15, 32].

Specializing to the evaluation of the function $p_{\text {ring }}^{\text {finite }}$, there is a further subtlety in the non- $n_{b}$ part that we have to take into account. As discussed in Sec. IIIF there is an unphysical pole in the integrand of Eq. (3.6) which implies that we cannot deform our integration path from the Euclidean to the Minkowskian axis quite as suggested by Eq. (D1). Instead, we have to try to avoid numerically dangerous singularities on the complex $q_{0}$ plane by encircling them at a safe distance. This aspect of our calculation is discussed in more detail below in Appendix D2a.

After all the separations, we can write the function $p_{\text {ring }}^{\text {finite }}$ as the sum of several individual contributions according to

$$
p_{\text {ring }}^{\text {finite }}=p_{1}^{\mathrm{E}}+p_{2}^{\mathrm{E}}+p_{\mathrm{L}}^{\mathrm{M}}+p_{\mathrm{LD}}^{\mathrm{M}}+p_{\mathrm{T}}^{\mathrm{M}}+p_{\mathrm{TD}}^{\mathrm{M}}+p_{m}^{\mathrm{M}} .
$$

Here, $p_{1}^{\mathrm{E}}$ and $p_{2}^{\mathrm{E}}$ denote two distinct parts of the Euclidean integral that are separated by a momentum cut-off $\Lambda$. The pieces $p_{\mathrm{L}}^{\mathrm{M}}$ and $p_{\mathrm{T}}^{\mathrm{M}}$ on the other hand stand for longitudinal and transverse Minkowskian contributions to $p_{\text {ring }}^{\text {finite }}$ integrated along specific segments of the real $q_{0}$-axis and $p_{\mathrm{LD}}^{\mathrm{M}}$ and $p_{\mathrm{TD}}^{\mathrm{M}}$ for the corresponding quantities integrated along deformed paths (see Fig. (D 2a $)$ ). In addition, $m$ refers to corrections due to the IR regulator mass introduced in Sec. III C that can be explicitly extracted from the rest. Each of these terms will be discussed in detail in the following.

\section{Euclidean space contributions}

For practical numerical reasons, we divide the Euclidean space "non- $n_{b}$ " contribution to Eq. (D3) into two parts $p_{1}^{\mathrm{E}}+p_{2}^{\mathrm{E}}$ by introducing a four-momentum cut-off $\Lambda$ and having the first piece

16 The quantities evaluated in Minkowskian metric $(+,-,-,-)$ (implying $Q^{2}=q_{0}^{2}-q^{2}$ ) are denoted by an upper index M, while Euclidean pieces, where $q_{0}$ is analytically continued back to Euclidean space $\left(q_{0} \rightarrow-i q_{0} \equiv \omega\right)$, are indicated by the use of the "Matsubara variable" $\omega$ and the upper index E. We have dropped the index $n$ from $\omega_{n}$ as it now has become a continuous variable. 
correspond to the contribution of momenta with $|Q|<\Lambda$. For this, we obtain by first performing the integration over an Euclidean four-sphere

$$
p_{1}^{\mathrm{E}}=-\frac{d_{A}}{2 \pi^{3}} \int_{0}^{\Lambda} d Q Q^{3} \int_{0}^{\pi / 2} d \alpha \sin ^{2} \alpha \operatorname{Re}\left(p_{\mathrm{int}}^{\mathrm{E}}(\omega, q)\right)
$$

with $\omega=-i q_{0}=Q \cos \alpha$ and $q=Q \sin \alpha$. The integrand $p_{\text {int }}^{\mathrm{E}}(\omega, q)$ is given by

$$
\begin{aligned}
p_{\mathrm{int}}^{\mathrm{E}}(\omega, q) & =\log \left[1+\frac{\Pi_{\mathrm{T}}+m_{\mathrm{mag}}^{2}}{q^{2}+\omega^{2}}\right]-\frac{\Pi_{\mathrm{T}}+m_{\mathrm{mag}}^{2}}{q^{2}+\omega^{2}}+\frac{\left(C_{A} g^{2} T^{2}-6 m_{\mathrm{mag}}^{2}\right)^{2}}{72\left(q^{2}+\omega^{2}+m^{2}\right)^{2}} \\
& +\frac{1}{2}\left(\log \left[1+\frac{\Pi_{\mathrm{L}}}{q^{2}+\omega^{2}}\right]-\frac{\Pi_{\mathrm{L}}}{q^{2}+\omega^{2}}+\frac{C_{A}^{2} g^{4} T^{4}}{72\left(q^{2}+\omega^{2}+m^{2}\right)^{2}}\right)
\end{aligned}
$$

where the magnetic mass $m_{\text {mag }}$, introduced in Eq. (3.24), is needed in the transverse part in order to prevent a negative argument of the logarithm as $\omega^{2}+q^{2} \rightarrow 0$. This provides a cutoff for IR divergences at the scale $m_{\text {mag }}=c_{f} \frac{3}{32} g^{2} C_{A} T$, with $c_{f} \geq 1$ being a "magnetic factor" that we can vary to verify that the dependence of the result on it is beyond $\mathcal{O}\left(g^{4}\right)$.

In addition to regulating IR divergencies, the inclusion of the magnetic mass alters the UV behavior of the first term of the above integrand which is why we included it also in the coefficient of the UV regulating last term of the first line of Eq. (D5). In order to keep the final result independent of the arbitrary mass parameter $m$, we have to take the magnetic mass $m_{\text {mag }}$ into account also in the calculation of the function $p_{\text {ring }}^{\mathrm{IR}}$ of Eqs. (3.7) and (B5), which amounts to replacing $C_{A}^{2}$ in those equations as well as later in Eqs. (D10) and (D15) by $C_{A}^{2}-8 g^{2} C_{A} \bar{m}_{\mathrm{mag}}^{2}+24 g^{4} \bar{m}_{\mathrm{mag}}^{4}$, as shown below in Eq. (D7).

\section{a. High momentum expansion in Euclidean space}

To obtain the high-momentum Euclidean contribution $p_{2}^{\mathrm{E}}$ to Eq. (D3) in the most effective way, we first integrate over $\alpha$, then expand $\Pi_{\mathrm{T}}$ and $\Pi_{\mathrm{L}}$ in the limit of large momenta (which is possible analytically order by order in $Q / T$ ) and finally perform the integration over $Q$. This produces a series of the general form

$$
p_{2}^{\mathrm{E}}=p_{\text {high }, 0}^{\mathrm{E}}+p_{\text {high }, 2}^{\mathrm{E}}+p_{\text {high }, 4}^{\mathrm{E}}+\ldots
$$

where the lowest orders are given by

$$
\begin{aligned}
p_{\mathrm{high}, 0}^{\mathrm{E}} & =-\frac{d_{A}}{768 \pi^{2}} g^{4} T^{4}\left(C_{A}^{2}-8 g^{2} C_{A} \bar{m}_{\mathrm{mag}}^{2}+24 g^{4} \bar{m}_{\mathrm{mag}}^{4}\right)\left(\log \frac{\Lambda^{2}}{m^{2}+\Lambda^{2}}-\frac{m^{2}}{m^{2}+\Lambda^{2}}\right), \\
p_{\mathrm{high}, 2}^{\mathrm{E}} & =-\frac{d_{A}}{3456 \pi^{2} \Lambda^{2}} g^{6} T^{6}\left\{-\frac{C_{A}^{3}}{2}-36 g^{4} C_{A} \bar{m}_{\mathrm{mag}}^{4}+72 g^{6} \bar{m}_{\mathrm{mag}}^{6}\right. \\
& \left.+12 \pi^{2} \bar{m}_{\mathrm{mag}}^{2}\left(\frac{g^{2} C_{A}^{2}}{2 \pi^{2}}+\frac{8 C_{A}+14 T_{F}}{5}+48 T_{F} \bar{\mu}^{2}+96 T_{F} \bar{\mu}^{4}\right)\right\}
\end{aligned}
$$

with $\bar{m}_{\text {mag }} \equiv m_{\text {mag }} /\left(g^{2} T\right)=c_{f} \frac{3}{32} C_{A}$ (with $\left.c_{f} \geq 1\right)$ and $\bar{\mu} \equiv \mu /(2 \pi T)$ as before. In our numerical implementation, we included terms up to $p_{\text {high,10 }}^{\mathrm{E}}$ and used a cut-off $\Lambda \sim 160 \sqrt{T^{2}+\mu^{2}}$, for which the error in the sum of $p_{1}^{\mathrm{E}}$ and $p_{2}^{\mathrm{E}}$ due to the introduction of the cut-off and the use of a high-momentum expansion in the second part is negligible. 


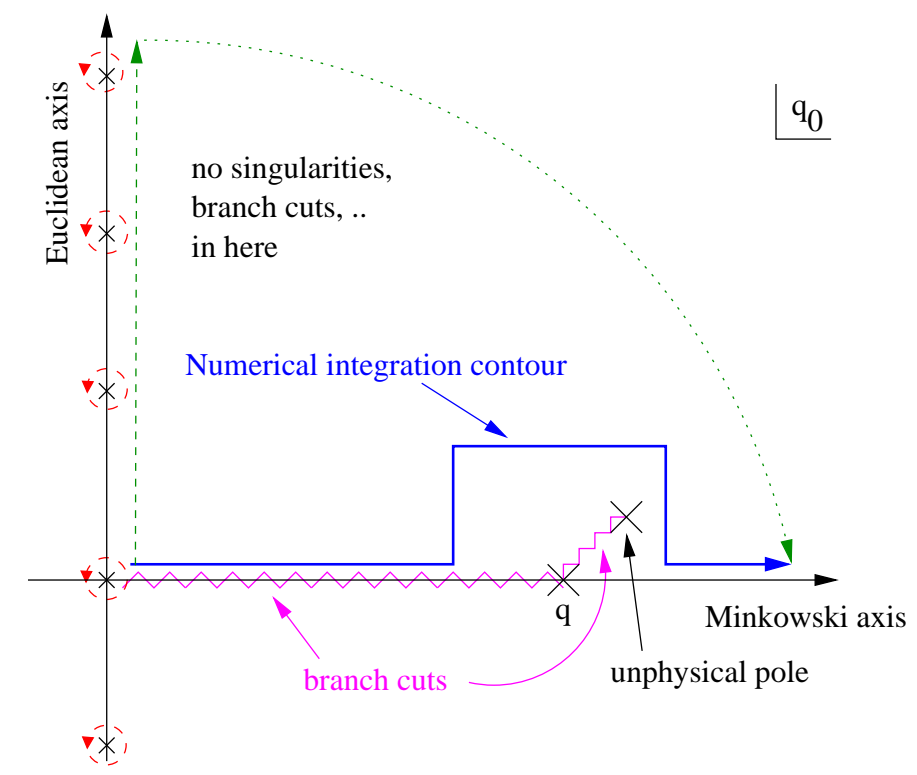

FIG. 18: Symbolic illustration of the analytic structure of the integrand of $p_{\text {ring }}^{\text {finite }}$ on the complex plane. The unphysical pole is avoided by using the complex integration path described in the text.

\section{b. IR mass in Euclidean space}

The IR regulating mass $m$ only appears in Eq. (3.6) in two terms that are independent of the self energies, and thus the difference of $p_{\text {ring }}^{\text {finite }}$ evaluated with masses $m_{0}$ and $m$ is given by

$$
p_{\text {ring }}^{\text {finite }}(m)-p_{\text {ring }}^{\text {finite }}\left(m_{0}\right)=-\frac{d_{A} C_{A}^{2} g^{4} T^{4}}{48} \sum_{P}\left\{\frac{1}{\left(P^{2}+m^{2}\right)^{2}}-\frac{1}{\left(P^{2}+m_{0}^{2}\right)^{2}}\right\} .
$$

From Eq. (Bי), we see that the Euclidean contribution to this sum-integral is

$$
p_{\text {ring }}^{\mathrm{fin}, \mathrm{E}}(m)-p_{\text {ring }}^{\mathrm{fin}, \mathrm{E}}\left(m_{0}\right)=\frac{d_{A} C_{A}^{2} g^{4} T^{4}}{24(4 \pi)^{2}} \log \frac{m}{m_{0}}
$$

which implies that the cancelation of $m$ in the sum of the Euclidean contributions to $p_{\text {ring }}^{\text {safe }}$ can be tested independently of the Minkowskian ones by verifying that the $m$ dependence of the numerical result for $p_{1}^{\mathrm{E}}+p_{2}^{\mathrm{E}}$ is exactly of the above form. We have done so with the expected positive result.

\section{Minkowski space contributions}

\section{a. Contour deformation}

As discussed in Sec. [IIF even after the introduction of the magnetic mass in $p_{\text {ring }}^{\text {finite }}$ there is an unphysical pole remaining on the complex $q_{0}$ plane which prohibits the use of the preferred " $n_{b}$ " integration path along the Minkowskian axis. Instead, we have to choose our integration contour so that we avoid the unphysical pole, illustrated in Fig. 18. Since a standard path along the Minkowskian axis would fail at the branch cut from $q_{0}=q$ to the unphysical pole, our numerical integration contour goes around the problematic zones at a safe distance, so that (given the vanishing great arc contribution) the final integration is equivalent to integrating along the Euclidean 


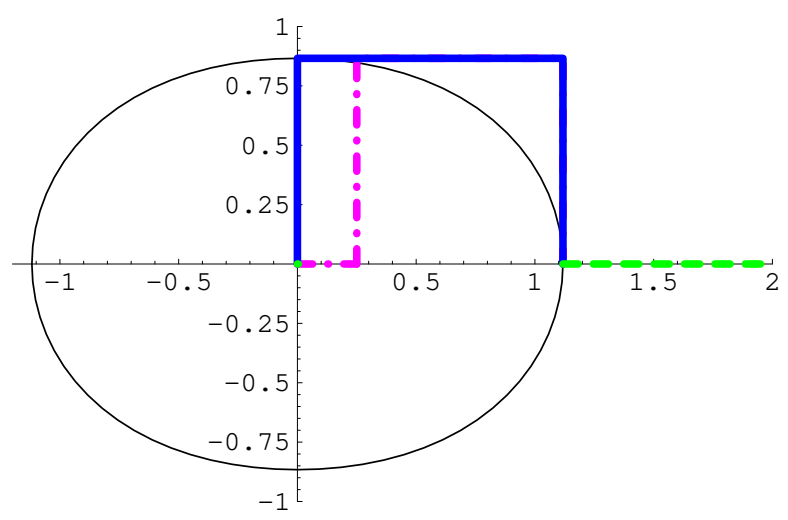

FIG. 19: The shape of the Debye mass cutoff and the numerical integration contour chosen on the complex $q_{0}$ plane, in units of $r$. The full line shows the deformed integration path along complex $q_{0}$ while the dotted line indicates usual Minkowski space integration, extending to $q_{0} \rightarrow \infty$. The dash-dotted line shows the integration path if it is restricted to stay in Minkowski space up to $q_{0}=q / 2$. The parameters in this and the following two Figures are chosen (a) for the big circle shape $(q=.5 r)$

axis - or just summing the discrete Matsubara frequencies we started from. The validity of this procedure can be checked afterwards by varying the safety distance between our integration path and the branch cuts.

The unphysical poles are expected to appear in the vicinity of the lightcone. We thus want our integration path to avoid the region $\left|q_{0}^{2}-q^{2}\right| \leq r^{2}$ where $r$ is chosen to be of the order of the Debye mass $r=f_{c} m_{\mathrm{D}}$ and $f_{c}>1$ is an arbitrary cutoff factor on which the final result should not depend. We will, however, not integrate exactly along this path, but instead choose a rectangular encasing boundary. To determine the shape of this optimal rectangular path, we write the condition

$$
\left|q_{0}^{2}-q^{2}\right|=\left|(a+i b)^{2}-q^{2}\right|<r^{2}
$$

in terms of the real quantities $a=\operatorname{Re} q_{0}$ and $b=\operatorname{Im} q_{0}$ which gives the relation

$$
4 a^{2} b^{2}+\left(a^{2}-b^{2}-q^{2}\right)^{2}<r^{4} .
$$

Solving for the extremal points for $a$ and $b$ from here and denoting $x \equiv \sqrt{r^{2}+q^{2}}, y \equiv \sqrt{\left|r^{2}-q^{2}\right|}$, $z \equiv r^{2} /(2 q)$, we find the three cases

$$
\begin{aligned}
& q<\frac{r}{\sqrt{2}}: \quad q_{0} \in\{0, i y, x+i y, x\} \quad \text { (big circle) } \\
& \frac{r}{\sqrt{2}} \leq q<r: \quad q_{0} \in\{0, i z, x+i z, x\} \quad \text { (eyeglasses) } \\
& q \geq r: q_{0} \in\{y, y+i z, x+i z, x\} \quad \text { (two circles) }
\end{aligned}
$$

where the notation $q_{0} \in\{u, v, w, z\}$ implies that the integration path on the complex $q_{0}$ plane proceeds along straight lines through these points. In practise, it is convenient to start the integration along the Minkowskian axis also in the two first cases, so from 0 to $q / 2$ the integration is always performed in Minkowski space. The three contours are depicted in Figs. 19- 21.

With the above integration paths, the notation in Eq. (D3) becomes obvious. The pieces $p_{\mathrm{L}}^{\mathrm{M}}$ and $p_{\mathrm{T}}^{\mathrm{M}}$ denote the integrations performed along the Minkowskian axis (dashed lines), making use of optimizations [32], while $p_{\mathrm{LD}}^{\mathrm{M}}$ and $p_{\mathrm{TD}}^{\mathrm{M}}$ are computed using the deformed paths (full lines or dash-dotted lines) for complex $q_{0}$. The Minkowskian contributions $p_{\mathrm{L}}^{\mathrm{M}}$ and $p_{\mathrm{T}}^{\mathrm{M}}$ are calculated as

$$
p_{\mathrm{L} / \mathrm{T}}^{\mathrm{M}}=-\frac{d_{A}}{2 \pi^{3}} \int_{0}^{\infty} d q q^{2} \int_{q_{0, \min }}^{\infty} d q_{0} \operatorname{Im}\left(p_{\mathrm{int}}^{\mathrm{M}}\left(q_{0}, q\right)\right)
$$




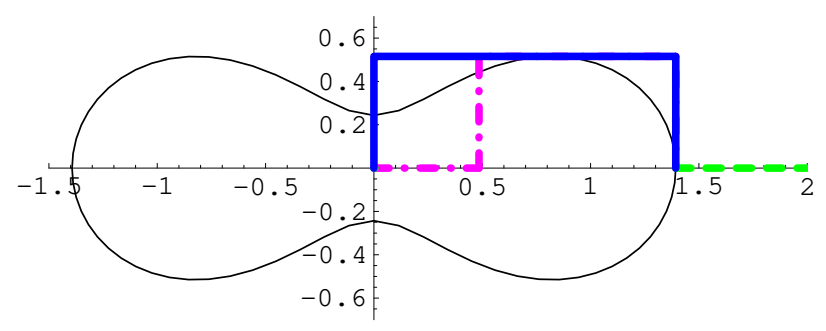

FIG. 20: (b) for the eyeglasses shape $(q=.97 r)$

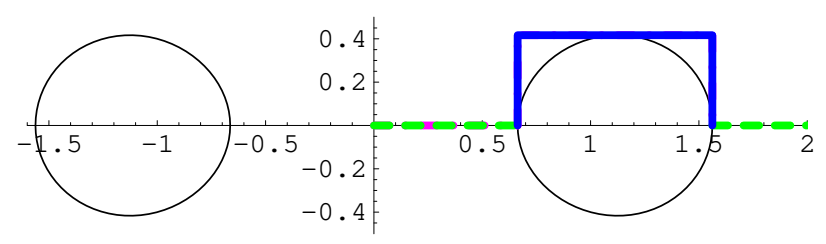

FIG. 21: and (c) for the two circles shape $(q=1.2 r)$.

where $q_{0, \text { min }}$ denotes the starting point of the green dashed lines in Figs. 19-21 (with the exception of the two circles shape for which the integration contour is composed of two disjoint pieces) and $p_{\text {int }}^{\mathrm{M}}$ is the straightforward analytic continuation of Eq. (D5) from Euclidean to Minkowski space. In Minkowski space, the Boltzmann factor $n_{b}$ provides a natural UV cutoff and the IR mass dependent term can be evaluated separately (see below).

The contribution of the deformed paths, $p_{\mathrm{LD}}^{\mathrm{M}}+p_{\mathrm{TD}}^{\mathrm{M}}$, is computed similarly as

$$
p_{\mathrm{LD} / \mathrm{TD}}^{\mathrm{M}}=-\frac{d_{A}}{2 \pi^{3}} \int_{0}^{\infty} d q q^{2} \int_{\text {Detour-path }} d q_{0} \operatorname{Im}\left(p_{\mathrm{int}}^{\mathrm{M}}\left(q_{0}, q\right)\right) .
$$

\section{b. IR mass in Minkowski space}

The so far unevaluated last contribution to Eq. (D3) is $p_{m}^{\mathrm{M}}$ which is the IR mass correction term corresponding to the $m$-dependent terms of Eq. (3.6). This integral can be computed separately from the other Minkowskian pieces, since the bosonic distribution function provides a natural UV cutoff for the Minkowski space calculations, making the subtraction of the quartic term from $p_{\text {ring }}^{\text {finite }}$ unnecessary. Writing $p_{m}^{\mathrm{M}}$ down explicitly (see the discussion below Eq. (D5) on how to include $\left.m_{\text {mag }}\right)$, we obtain

$$
p_{m}^{\mathrm{M}}=\frac{d_{A} C_{A}^{2} g^{4} T^{4}}{24} \int_{0}^{\infty} \frac{d q_{0}}{\pi} \int \frac{d^{3} q}{(2 \pi)^{3}} n_{b} \operatorname{Im} \frac{1}{\left(Q^{2}-m^{2}\right)^{2}}
$$

which, not surprisingly, is observed to exactly cancel the third term of Eq. (B5). Thus we see that all dependence on the regulator mass $m$ cancels between the different Minkowskian contributions to $p_{\text {ring }}^{\text {safe }}$ which together with our observation after Eq. (D10) explicitly verifies the independence of 
our final result $p_{\mathrm{IV}}$ of $m$.

[1] E. V. Shuryak, Sov. Phys. JETP 47 (1978) 212 [Zh. Eksp. Teor. Fiz. 74 (1978) 408]. S. A. Chin, Phys. Lett. B 78 (1978) 552.

[2] J. I. Kapusta, Nucl. Phys. B 148 (1979) 461.

[3] T. Toimela, Phys. Lett. B 124 (1983) 407.

[4] P. Arnold and C. X. Zhai, Phys. Rev. D 50 (1994) 7603 hep-ph/9408276; Phys. Rev. D 51 (1995) 1906 hep-ph/9410360.

[5] C. X. Zhai and B. Kastening, Phys. Rev. D 52 (1995) 7232 hep-ph/9507380.

[6] E. Braaten and A. Nieto, Phys. Rev. D 53 (1996) 3421 hep-ph/9510408.

[7] K. Kajantie, M. Laine, K. Rummukainen and Y. Schröder, Phys. Rev. D 67 (2003) 105008 hep-ph/0211321.

[8] A. Vuorinen, Phys. Rev. D 68 (2003) 054017 hep-ph/0305183.

[9] B. A. Freedman and L. D. McLerran, Phys. Rev. D 16 (1977) 1169; V. Baluni, Phys. Rev. D 17 (1978) 2092.

[10] A. Ipp, A. Gerhold and A. Rebhan, Phys. Rev. D 69 (2004) 011901 hep-ph/0309019.

[11] A. Roberge and N. Weiss, Nucl. Phys. B 275 (1986) 734.

[12] J. C. Taylor and S. M. H. Wong, Nucl. Phys. B 346, 115 (1990); E. Braaten and R. D. Pisarski, Phys. Rev. D 45, 1827 (1992).

[13] D. T. Son, Phys. Rev. D 59 (1999) 094019 hep-ph/9812287.

[14] A. Gynther, Phys. Rev. D 68 (2003) 016001 hep-ph/0303019.

[15] A. Ipp and A. Rebhan, JHEP 0306 (2003) 032 hep-ph/0305030.

[16] A. Gerhold, A. Ipp and A. Rebhan, Phys. Rev. D 70 (2004) 105015 hep-ph/0406087.

[17] A. Vuorinen, "The pressure of QCD at finite temperature and quark number density," $\mathrm{PhD}$ thesis hep-ph/0402242.

[18] M. Laine and Y. Schröder, "Quark mass thresholds in QCD thermodynamics," hep-ph/0603048

[19] E. S. Fraga and P. Romatschke, Phys. Rev. D 71, 105014 (2005) hep-ph/0412298.

[20] A. Hart, M. Laine and O. Philipsen, Nucl. Phys. B 586, 443 (2000) hep-ph/0004060.

[21] C. P. Korthals Altes, R. D. Pisarski and A. Sinkovics, Phys. Rev. D 61, 056007 (2000) hep-ph/9904305.

[22] J. P. Blaizot, E. Iancu and A. Rebhan, Phys. Lett. B 523, 143 (2001) hep-ph/0110369.

[23] T. Holstein, R. E. Norton, and P. Pincus, Phys. Rev. B8, 2649 (1973).

[24] A. Gerhold and A. Rebhan, Phys. Rev. D 71, 085010 (2005) hep-ph/0501089.

[25] J. O. Andersen, E. Braaten, E. Petitgirard, and M. Strickland, Phys. Rev. D66, 085016 (2002) hep-ph/0205085; J. O. Andersen, E. Petitgirard, and M. Strickland, Phys. Rev. D70, 045001 (2004) hep-ph/0302069.

[26] J. P. Blaizot, E. Iancu, and A. Rebhan, Phys. Rev. Lett. 83, 2906 (1999) hep-ph/9906340; Phys. Lett. B470, 181 (1999) hep-ph/9910309; Phys. Rev. D63, 065003 (2001) hep-ph/0005003.

[27] E. S. Fraga, R. D. Pisarski and J. Schaffner-Bielich, Phys. Rev. D 63, 121702 (2001) hep-ph/0101143.

[28] A. D. Linde, Phys. Lett. B 96, 289 (1980).

[29] O. K. Kalashnikov and V. V. Klimov, Sov. J. Nucl. Phys. 33, 443 (1981) [Yad. Fiz. 33, 848 (1980)].

[30] O. K. Kalashnikov, Fortsch. Phys. 32, 525 (1984).

[31] U. Kraemmer and A. Rebhan, Rept. Prog. Phys. 67, 351 (2004) hep-ph/0310337.

[32] G. D. Moore, JHEP 0210 (2002) 055 hep-ph/0209190; A. Ipp, G. D. Moore and A. Rebhan, JHEP 0301 (2003) 037 hep-ph/0301057.

[33] J. Kapusta, "Finite temperature field theory", Cambridge University Press (1989).

[34] O. K. Kalashnikov and V. V. Klimov, Sov. J. Nucl. Phys. 31, 699 (1980) [Yad. Fiz. 31, 1357 (1980)].

[35] K. Kajantie and J. I. Kapusta, Annals Phys. 160, 477 (1985).

[36] U. W. Heinz, K. Kajantie and T. Toimela, Annals Phys. 176, 218 (1987).

[37] E. Braaten and R. D. Pisarski, Phys. Rev. D 42, 2156 (1990).

[38] T. Schäfer and K. Schwenzer, Phys. Rev. D 70, 054007 (2004) arXiv:hep-ph/0405053.

[39] A. Schmitt, Phys. Rev. D 71, 054016 (2005) nucl-th/0412033.

[40] W. E. Brown, J. T. Liu and H. c. Ren, Phys. Rev. D 61, 114012 (2000) arXiv:hep-ph/9908248; 
Phys. Rev. D 62, 054013, 054016 (2000) arXiv:hep-ph/9912409,0003199]; A. Schmitt, Q. Wang and D. H. Rischke, Phys. Rev. D 66, 114010 (2002) arXiv:nucl-th/0209050.

[41] J. P. Blaizot, E. Iancu and A. Rebhan, Phys. Rev. D 68, 025011 (2003) hep-ph/0303045.

[42] A. Ipp, A. Rebhan and A. Vuorinen, Phys. Rev. D 69, 077901 (2004) hep-ph/0311200.

[43] J. P. Blaizot, A. Ipp and A. Rebhan, "Study of the gluon propagator in the large- $N_{f}$ limit at finite temperature and chemical potential for weak and strong couplings," Annals of Physics (in print) hep-ph/0508317.

[44] A. Vuorinen, Phys. Rev. D 67 (2003) 074032 hep-ph/0212283.

[45] H. A. Weldon, Phys. Rev. D 26, 1394 (1982). 